Final Technical Report

U.S. Department of Energy

\title{
Reactive Membrane Barriers for Containment of Subsurface Contamination
}

\author{
Principal Investigators \\ William A. Arnold \\ Department of Civil Engineering \\ University of Minnesota \\ Minneapolis, MN 55455 \\ and \\ Edward L. Cussler \\ Department of Chemical Engineering and Materials Science \\ University of Minnesota \\ Minneapolis, MN 55455 \\ Project Number: 86800 \\ Grant Number: DE-FG07-02ER63509 \\ Duration: 9/15/2002-12/15/2006
}




\section{TABLE OF CONTENTS}

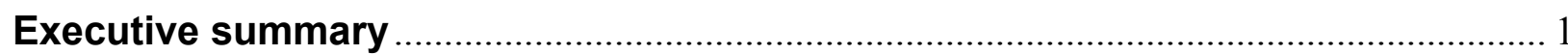

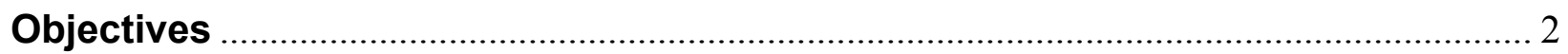

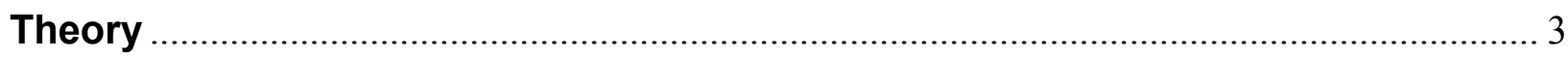

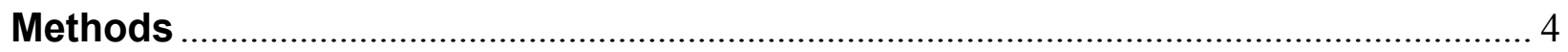

Poly(vinyl alcohol) Membrane Preparation............................................................................. 4

High Density Polyethylene Membrane Preparation ................................................................. 5

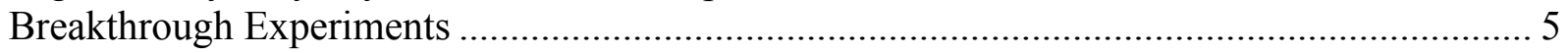

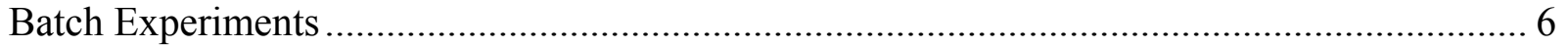

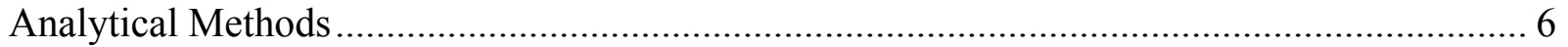

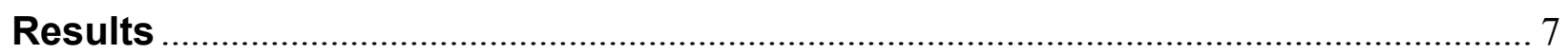

A PVA Membrane Containing $\mathrm{Fe}^{0}$ as a Contaminant Barrier .................................................. 7

Permeable Membranes Containing Crystalline Silicotitanate As Model Barriers For Cesium

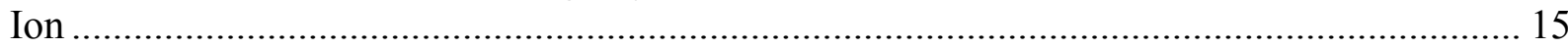

Model membrane performance using Hanford Groundwater................................................. 21

High-Density Polyethylene Membrane Containing $\mathrm{Fe}^{0}$ as a Contaminant Barrier .................. 25

The Third Parameter in Reactive Barrier Films................................................................. 30

Diffusion of Mobile Products in Reactive Barrier Membranes .................................................. 42

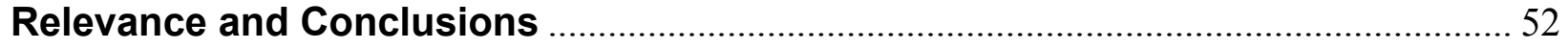

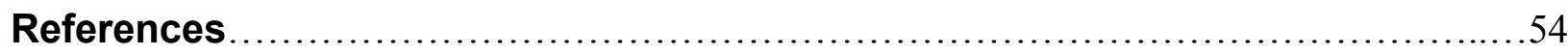

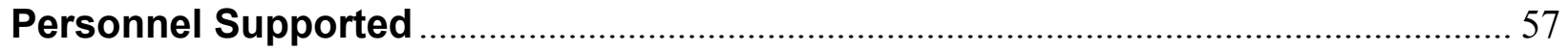

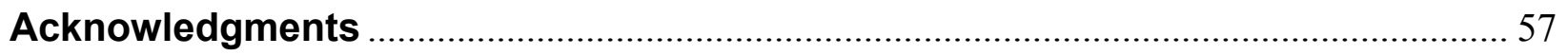

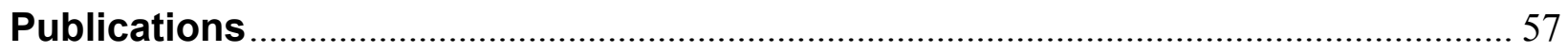

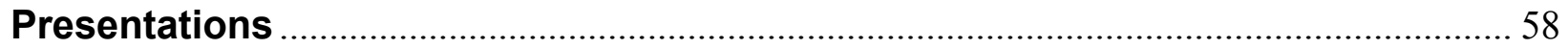




\section{Executive summary}

The overall goal of this project was to develop reactive membrane barriers--a new and flexible technique to contain and stabilize subsurface contaminants. Polymer membranes will leak once a contaminant is able to diffuse through the membrane. By incorporating a reactive material in the polymer, however, the contaminant is degraded or immobilized within the membrane. These processes increase the time for contaminants to breakthrough the barrier (i.e. the lag time) and can dramatically extend barrier lifetimes. In this work, reactive barrier membranes containing zero-valent iron $\left(\mathrm{Fe}^{0}\right)$ or crystalline silicotitanate (CST) were developed to prevent the migration of chlorinated solvents and cesium-137, respectively. These studies were complemented by the development of models quantifying the leakage/kill time of reactive membranes and describing the behavior of products produced via the reactions within the membranes.

First, poly(vinyl alcohol) (PVA) membranes containing $\mathrm{Fe}^{0}$ and CST were prepared and tested. Although PVA is not useful in practical applications, it allows experiments to be performed rapidly and the results to be compared to theory. For copper ions $\left(\mathrm{Cu}^{2+}\right)$ and carbon tetrachloride, the barrier was effective, increasing the time to breakthrough over 300 times. Even better performance was expected, and the percentage of the iron used in the reaction with the contaminants was determined. For cesium, the CST laden membranes increased lag times more than 30 times, and performed better than theoretical predictions. A modified theory was developed for ion exchangers in reactive membranes to explain this result. With the PVA membranes, the effect of a groundwater matrix on barrier performance was tested. Using Hanford groundwater, the performance of $\mathrm{Fe}^{0}$ barriers decreased compared to solutions containing a $\mathrm{pH}$ buffer and high levels of chloride (both of which promote iron reactivity). For the CST bearing membrane, performance improved by a factor of three when groundwater was used in place of deionized water.

The performance of high density polyethylene (HDPE) membranes containing $\mathrm{Fe}^{0}$ was then evaluating using carbon tetrachloride as the target contaminant. Only with a hydrophilic additive (glycerol), was the iron able to extend lag times. Lag times were increased by a factor of 15 , but only $2-3 \%$ of the iron was used, likely due to formation of oxide precipitates on the iron surface, which slowed the reaction. With thicker membranes and lower carbon tetrachloride concentrations, it is expected that performance will improve.

Previous models for reactive membranes were also extended. The lag time is a measurement of when the barrier is breached, but contaminants do slowly leak through prior to the lag time. Thus, two parameters, the leakage and the kill time, were developed to determine when a certain amount of pollutant has escaped (the kill time) or when a given exposure (concentration $\times$ time) occurs (the leakage). Finally, a model was developed to explain the behavior of mobile reaction products in reactive barrier membranes. Although the goal of the technology is to avoid such products, it is important to be able to predict how these products will behave. Interestingly, calculations show that for any mobile reaction products, one half of the mass will diffuse into the containment area and one half will escape, assuming that the volumes of the containment area and the surrounding environment are much larger than the barrier membrane. These parameters/models will aid in the effective design of barrier membranes. 


\section{Objectives}

Contaminants of concern to the U.S. Department of Energy include radionuclides (e.g., cesium-137, strontium-90), metals (e.g., chromium VI, $\mathrm{CrO}_{4}{ }^{2-}$ ), and chlorinated solvents (e.g., carbon tetrachloride (CT), tetrachloroethylene (PCE), trichloroethylene (TCE)), all of which are present at a various sites across the DOE complex. The contaminants are found in both the vadose and saturated zones. Chlorinated solvents may also be present above their aqueous solubility, leading to the presence of dense non-aqueous phase liquids (DNAPLs). Thus, it is desirable to have a containment or stabilization technology that can be used in a variety of situations for a variety of contaminants.

In some, if not many situations, contaminated soils cannot be excavated, or economically feasible remediation techniques do not exist because the contamination is too concentrated or the contaminated zone is too large or deep. In these instances, containment and/or stabilization may be the only option available. Moreover, temporary containment is often necessary even when remediation of the contaminants is planned because time is needed to evaluate and design the necessary treatment options. Finally, contaminant sources may localized (e.g, a DNAPL pool) or diffuse, necessitating a versatile technology that is useful for both concentrated and dilute contaminant sources. Even if meant to be temporary, it is advantageous for the system to have a long expected lifetime, for design and installation of remediation plans may take longer than expected or temporary containment strategies can become permanent ones because of budgetary or emphasis shifts.

Reactive membrane barriers meet all of the above criteria. Any "impermeable" membrane barrier (e.g., an HDPE geomembrane) used to contain or stabilize subsurface contamination will eventually leak due to diffusion of the contaminants through the polymer. Reactive membrane barriers, however, can degrade or immobilize contaminants within the membrane. These processes increase the time for contaminants to breakthrough the barrier and can dramatically extend barrier lifetimes. These barriers can be used to prevent vapor transport in the vadose zone, contaminated water transport in the saturated zone, and have the potential to contain DNAPLs as well.

The overall goal of this project was to develop reactive membrane barriers--a new and flexible technique to contain and stabilize subsurface contaminants. Because treatment/remediation at some sites is neither technically or economically feasible, containment or stabilization may be the only viable alternative. For containment to be effective, the barrier must prevent the transport/diffusion of the contaminant for as long as possible. Reactive membrane barriers will prevent the migration/breakthrough of contaminants for 100-1000 times longer than polymer membranes alone. These modified membranes have the potential to contain subsurface sources of organic and inorganic contaminants in both vadose and saturated zones. The transport of non-aqueous phase liquids (NAPLs) will also be prevented by these membranes. Two reactive particles were incorporated into polymer membranes: iron metal $(\mathrm{Fe}(0))$ and crystalline silicotitanate (CST). The $\mathrm{Fe}(0)$-containing membranes can be used to contain chlorinated solvents or chromate $\left(\mathrm{CrO}_{4}{ }^{2-}\right)$ sources in the subsurface by serving as an electron donor for their reductive transformation with in the membrane. CST is an ion exchange material with high selectivity for cesium and strontium. The reactive membranes represent a significant advance over current membrane-based containment techniques. 
The specific objectives of the project were to manufacture, test, and model the performance of the reactive membrane barriers described above. Specific tasks were to:

1. Manufacture and test polyvinyl alcohol (PVA) membranes containing reactive particles (iron metal or CST).

2. Manufacture and test high density polyethylene (HDPE) membranes containing reactive particles (iron metal).

3. Test the performance of the membranes with groundwater collected from one or more DOE sites to determine whether interference by components in the groundwater matrix occurs.

4. Use mathematical and/or numerical models to develop a set of analytical guidelines that facilitate the development of reactive barrier films to allow their application at contaminated DOE sites.

\section{Theory}

The expected increases in breakthrough or lag time for reactive membranes are based on equations developed previously and are briefly reviewed here. The theory for diffusion through a membrane without reaction predicts that the downstream concentration changes as (Crank, 1975; Yang et al. 2001):

$$
\begin{aligned}
& \frac{C_{\text {down }}}{C_{\text {up }}}=\frac{P A}{L V_{\text {down }}}\left(t-t_{\text {lag }}\right) \\
& \text { where } t_{\text {lag }}=\frac{L^{2}}{6 D}
\end{aligned}
$$

and where $C_{\text {down }}$ and $C_{u p}$ are the contaminant concentrations downstream and upstream of the membrane, respectively; $D$ is the diffusion coefficient for the selected contaminant in the membrane; $P$ is permeability of the membrane to the contaminant, equal to $H \cdot D ; H$ is the membrane-water partition coefficient for the contaminant into the membrane; $L$ is the membrane thickness; $V_{\text {down }}$ is the volume of the downstream compartment; $A$ is the cross-sectional area of the membrane available for diffusion; and $t$ and $t_{\text {lag }}$ are time and breakthrough lag time, respectively. Equations 1 and 2 are derived assuming that $C_{u p}$ is constant and that the volume of the upstream compartment is very large. Similar equations for other assumptions are easily derived.

For a membrane containing a reactive material that consumes contaminants irreversibly, the corresponding equation again has the form of eq 1 but with a different expression for the lag time (Yang et al., 2001):

$$
t_{\text {lag }}=\frac{L^{2} C_{0}}{2 v P C_{u p}}
$$

where $C_{0}$ is the initial concentration of the reactive material, and $v$ is the stoichiometric coefficient of the reaction. Equation 3 assumes an instantaneous reaction, i.e. the reaction is much faster than diffusion through the membrane. Yang et al. demonstrated that the breakthrough lag time for various acids through membranes containing $\mathrm{ZnO}$ obeyed eq 3 . Ideal breakthrough curves for membranes with and without a reactive material are illustrated in Figure 1. In general, eqs 1 through 3 are approximations, valid at small times before 
much contaminant penetration has occurred. These small times are important from an environmental viewpoint, as they allow predictions of initial contaminant fluxes through the barrier material. At longer times, the downstream concentration does not follow these relations, but becomes non-linear, as shown in Figure 1. Equations 1 through 3 form the basis for analyzing our experiments as described below. Extensions to this theory developed as part of this project are described in later sections.

\section{Methods}

\section{Poly(vinyl alcohol) Membrane Preparation}

Iron containing PVA membranes were prepared in an anaerobic chamber to prevent unwanted oxidation of $\mathrm{Fe}^{0}$. For membranes containing CST or zinc oxide (ZnO), this was not required. Nanoparticles of $\mathrm{Fe}^{0}$ were synthesized by mixing $0.1 \mathrm{M}$ aqueous solutions of

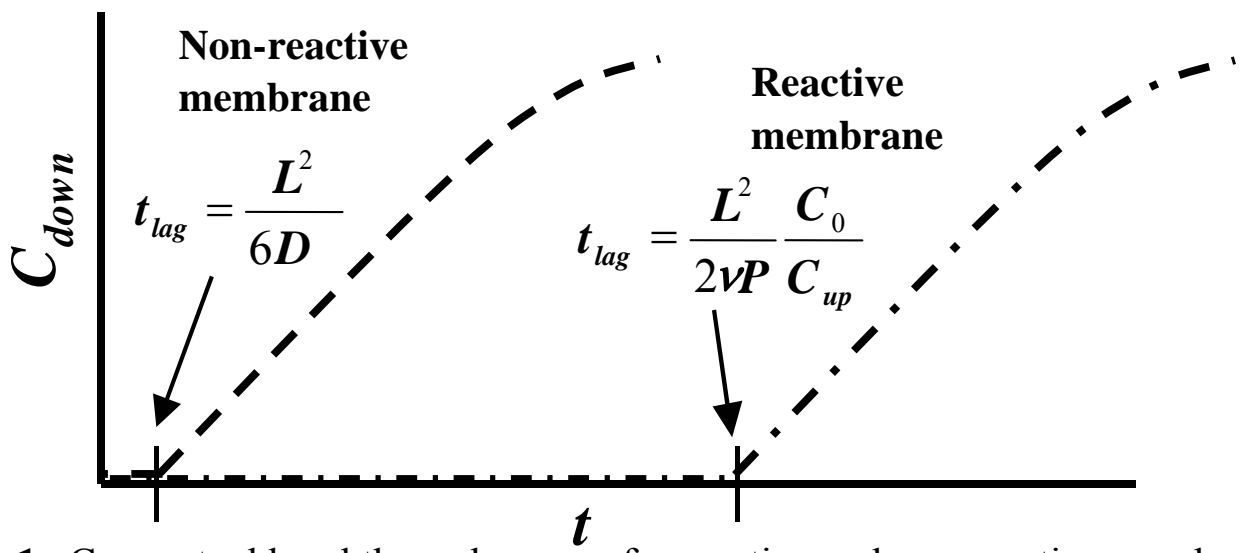

Figure 1. Conceptual breakthrough curves for reactive and non-reactive membranes. $C_{\text {down }}=$ downstream concentration of a contaminant, $t=$ time, $t_{\text {lag }}=$ breakthrough lag time, $L=$ membrane thickness, $D=$ diffusion coefficient of the contaminant in the membrane, $C_{0}=$ concentration of a reactive material in the membrane at the beginning of a diffusion experiment, $C_{u p}=$ upstream concentration of a contaminant, $v=$ stoichiometric coefficient of the reaction, and $P=$ permeability of the membrane for the specific contaminant.

$\mathrm{FeCl}_{3} \cdot 6 \mathrm{H}_{2} \mathrm{O}$ and $\mathrm{NaBH}_{4}$ as described previously (Wang and Zhang 1997). Minute, black particles were produced. A study has shown that nanoparticles of $\mathrm{Fe}^{0}$ synthesized in a similar manner contained approximately $4 \%$ boron by mass (Schrick et al., 2002), but we simply refer to the nanoparticles synthesized here as nanoparticles of $\mathrm{Fe}^{0}$. After the reaction was complete, the particles were rinsed with deoxygenated water to remove any residual $\mathrm{BH}_{4}{ }^{-}$and $\mathrm{BO}_{3}{ }^{3-}$ that could disrupt cross-linking of the PVA.

A PVA solution was prepared by adding $2.5 \mathrm{~g}$ of PVA to $30 \mathrm{~mL}$ of water. The mixture was heated to near boiling while stirring until a completely transparent solution was obtained. After the solution was cooled and degassed under vacuum, nanoparticles of $\mathrm{Fe}^{0}, \mathrm{CST}$, or $\mathrm{ZnO}$ were mixed into the solution. The mixture was cast on a smooth Teflon block, leveled with a doctor blade, and allowed to dry. After drying, the membrane was heated at $150{ }^{\circ} \mathrm{C}$ in an oven flushed with $\mathrm{N}_{2}$ gas for 1 hour to lightly crosslink the polymer. The pure PVA membranes were synthesized in a similar manner but without the addition of the immobilized reagent. Iron content 
in each synthesized $\mathrm{Fe}^{0}$ /PVA membrane was determined by the FerroZine method (Stookey, 1970) after dissolving the iron particles from the membrane with Aqua Regia (Sancar et al., 2000). The surfaces of $\mathrm{Fe}^{0} / \mathrm{PVA}$ membranes observed by SEM (Figure 2) show clusters of $\mathrm{Fe}^{0}$ evenly scattered in the membrane. The clusters are $1-2 \mu \mathrm{m}$ in diameter, and each cluster consists of smaller $\mathrm{Fe}^{0}$ particles (100-200 $\mathrm{nm}$ in diameter). The membrane thickness was measured after overnight hydration using a micrometer. Local variation of the membrane thickness was typically $\pm 10 \%$, and the average value of five measurements per membrane sample is reported. These membranes are much thinner than typical landfill liners, which are more than $1.5 \mathrm{~mm}$ thick (USEPA, 1994).
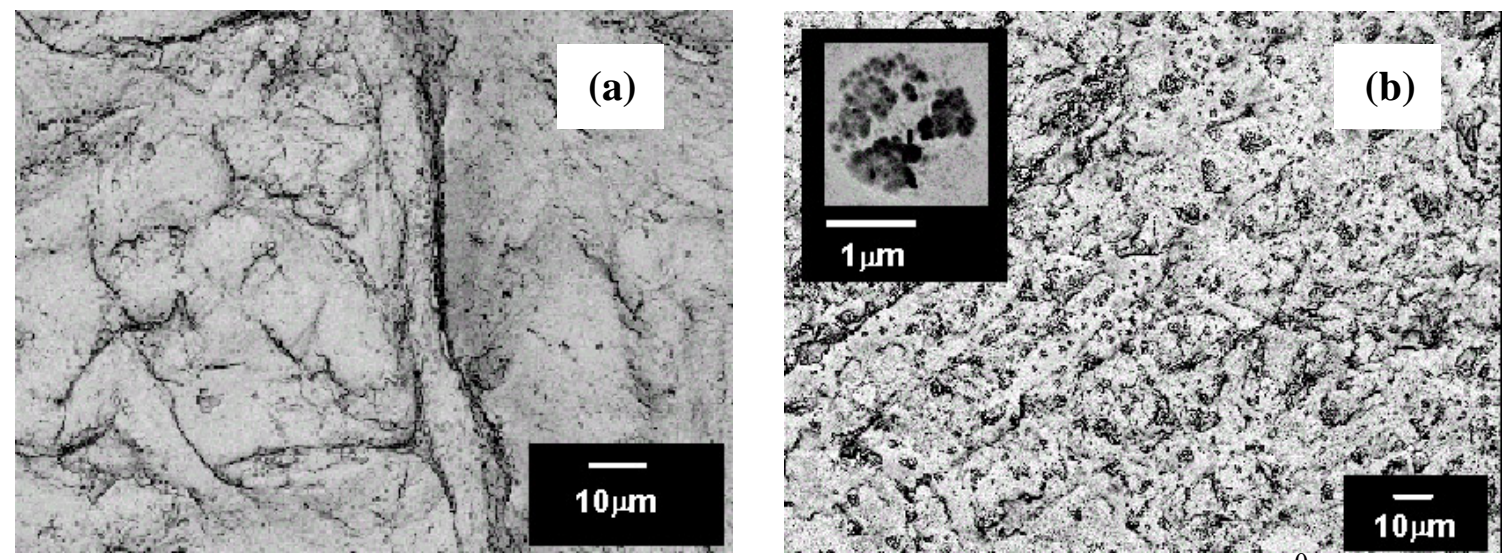

Figure 2. SEM images of (a) a dry PVA membrane surface and (b) a dry $\mathrm{Fe}^{0} / \mathrm{PVA}$ membrane surface before the breakthrough experiment. Black spots are nanoparticles of $\mathrm{Fe}^{0}$. Surface roughness is also observed as ridges. Images were inverted and adjusted for brightness and contrast.

\section{High Density Polyethylene Membrane Preparation}

The $\mathrm{Fe}^{0}$ nanoparticles described above were used. To blend the HDPE and $\mathrm{Fe}^{0}$ particles, a batch mixer was used. First, polymer (14-19 g) was transferred into the mixer and melted at 160 ${ }^{\circ} \mathrm{C}$. Iron nanoparticles $(1.0-3.5 \mathrm{~g})$ were then mixed into the molten polymer and blended for 10 min. The mixture was taken out of the mixer and pressed by a hydraulic molding press for $8 \mathrm{~min}$ at $150{ }^{\circ} \mathrm{C}$ and $275 \mathrm{kPa}$. The resulting membrane appeared uniformly black. Polymer membranes without $\mathrm{Fe}^{0}$ were produced by simply pressing polymer beads without the mixing process.

The $\mathrm{Fe}^{0}$ was transferred into the mixer via two methods to limit oxidation of the particles. In one method, the mixer and press were covered by a plastic bag and flushed with $\mathrm{N}_{2}$ to prevent oxidation. This method was used to prepare $\mathrm{Fe}^{0} / \mathrm{HDPE}$ membranes with no additive. For the second method, $\mathrm{Fe}^{0}$ particles were coated with glycerol (approximately two parts glycerol to one part iron by mass), making a black paste, before introduction to the mixer. The coating prevented the oxidation of $\mathrm{Fe}^{0}$ during its brief exposure to air, and therefore, the flushing with $\mathrm{N}_{2}$ was not necessary. This method was adopted to prepare $\mathrm{Fe}^{0} / \mathrm{HDPE}$ membranes with glycerol as an additive.

\section{Breakthrough Experiments}

Solute breakthrough across each membrane was tested with a diaphragm-cell diffusion apparatus as shown in Figure 3. Both cells were filled with the desired ( $\mathrm{pH}$ buffered) solution, 
and each cell was stirred with a Teflon-coated magnetic stirbar. Tests with colored dye showed complete mixing of solute in the cells within 15 seconds The target contaminant was introduced into the upstream cell to begin an experiment. The upstream cell had a larger volume so that the concentration in this cell changed minimally. The downstream concentrations were monitored by collection of aqueous samples or monitoring with an electrode. Samples were also collected from the upstream cell when desired. For PVA membranes, the membrane was held in place by clamping the two cells together. For HDPE, the membrane was glued to each cell using epoxy and the clamp was used for additional support.

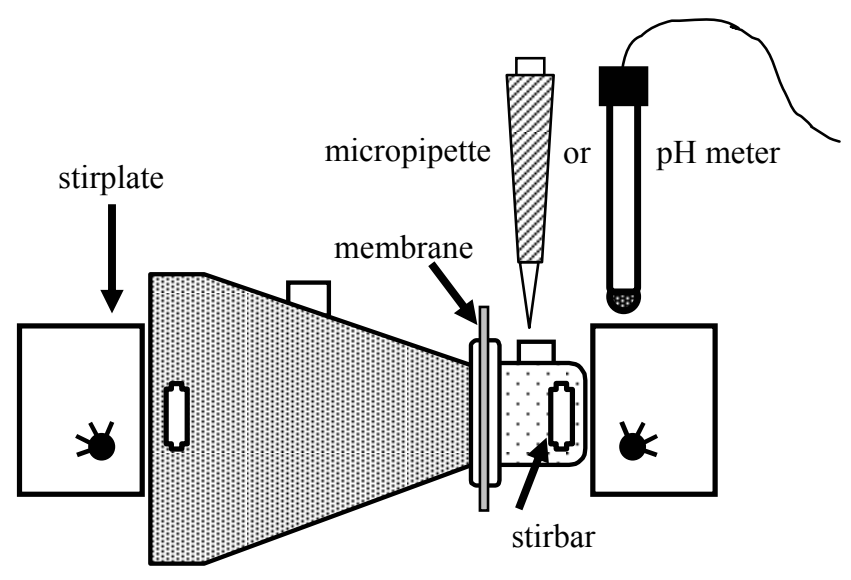

Figure 3. Schematic of a typical diaphragm cell used in this study. The larger cell is the 'upstream' cell initially spiked with the contaminant of interest.

\section{Batch Experiments}

The procedure for a typical sorption experiment was explained by Joo et al. (2004) in detail. In our study, a 123-mL serum bottle was loaded with pieces of HDPE membrane and filled with Milli-Q water leaving no headspace. CT was injected, and the bottle was capped with a Teflon-faced septum. At each sampling, an $10-\mu \mathrm{L}$ aqueous sample was withdrawn from the bottle. The experiments were run for up to 30 days, and the isotherm was created using average concentrations determined once solution concentrations were no longer changing ( 7 days or longer).

\section{Analytical Methods}

Analytical methods depended on the particular solute. Carbon tetrachloride was analyzed via gas chromatography with electron capture detection after extraction using pentane. The degradation products in selected samples were quantified with a flame ionization detector. Concentrations of $\mathrm{Cu}^{2+}, \mathrm{CrO}_{4}{ }^{2-}$, and $\mathrm{Zn}^{2+}$ were determined by the bathocuproine method, the diphenylcarbazide method, and the Zincon method respectively, using a UV-visible spectrophotometer (APHA, 1998). Nitrobenzene and 4-nitroacetophenone were measured via HPLC. Cesium, chloride, and proton $(\mathrm{pH})$ concentrations were measured using ion selective electrodes. Dissolved iron was quantified using the FerroZine method (Stookey et al., 1970). 


\section{Results}

\section{A PVA Membrane Containing $\mathrm{Fe}^{0}$ as a Contaminant Barrier}

\section{Pure PVA membranes}

We first present diffusion of a solute through pure PVA membranes. Note that all "pure" PVA membranes are hydrated and are $50 \%$ water by volume. Carbon tetrachloride, selected as a model contaminant, shows typical breakthrough curves for two membranes of different thickness in Figure 4. These curves are characterized by a slope and an intercept. The slopes equal

$\frac{P A}{L V_{\text {down }}}$, which is a measure of the steady-state flux of carbon tetrachloride. If these slopes are normalized by $A$ and $V_{\text {down }}$, the permeance, $\frac{P}{L}$, is calculated. The results in Figure 5 show that this permeance is roughly proportional to the reciprocal of the membrane thickness, supporting the analysis in eq 1. Possible reasons for the scatter of the data are stretching of the membrane, local variation of membrane thickness, and undetected pinholes. The thickness of most of the membranes ranges from 100-200 $\mu \mathrm{m}$. Within this span, it is easiest to synthesize intact membranes. Although a wider range of thickness would be desirable for the regression, thin membranes tend to have the problems mentioned above.

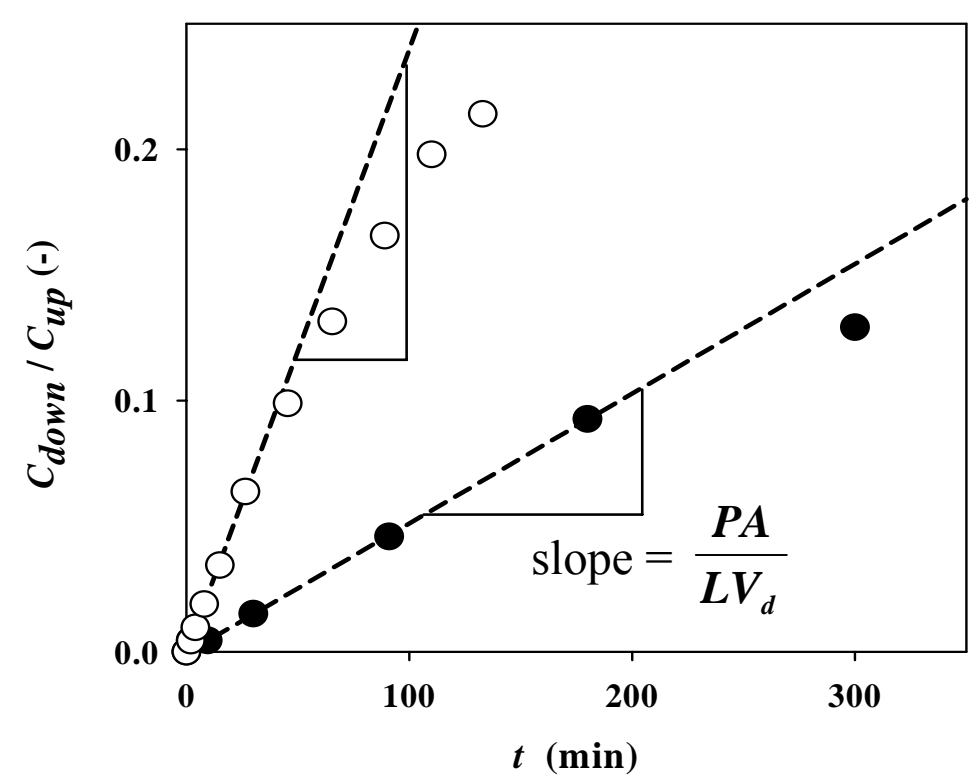

Figure 4. Typical breakthrough curves for carbon tetrachloride with pure PVA membranes. The downstream concentration $\left(C_{\text {down }}\right)$ is normalized by the initial upstream concentration $\left(C_{\text {up }}\right)$. $V_{\text {down }}$ and $A$ are the downstream cell volume and the membrane cross-sectional area available for diffusion, respectively. The two experiments $\left(\bullet, L=198 \mu \mathrm{m}, C_{u p}=146 \mu \mathrm{M}, V_{\text {down }}=16.64 \mathrm{~mL}\right.$; ○, $L=44 \mu \mathrm{m}, C_{u p}=131 \mu \mathrm{M}, V_{\text {down }}=13.11 \mathrm{~mL}$ ) used membranes with different thicknesses. The regressed, dashed lines are related to the steady-state flux. 


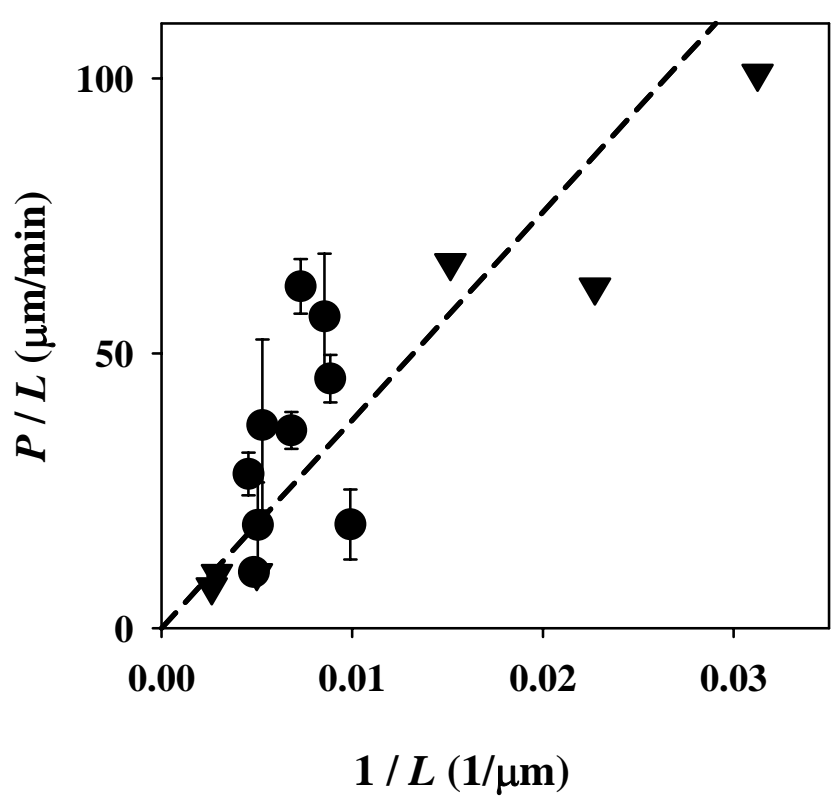

Figure 5. Variation of permeance with reciprocal membrane thickness. Error bars associated with the data points $(\bullet)$ indicate the standard deviation based on replicates using the same membrane. The data points without error bars $(\boldsymbol{\nabla})$ were not replicated. The dashed line represents a linear regression with zero intercept $\left(R^{2}=0.93\right)$.

Thicker membranes are also difficult to prepare, and calculations suggest the strong partitioning of carbon tetrachloride into the PVA (and the resulting loss of mass balance in the closed cell apparatus) may bias determination of the apparent permeability. The $\mathrm{x}$-intercepts in Figure 4 are the lag times, expected from eq 1 to be proportional to the square of the membrane thickness. Although the lag times show a systematic increase with increasing membrane thickness, they are too small $(<2 \mathrm{~min})$ to be precisely determined for most non-reactive membranes used in this study.

\section{$\mathrm{Fe}^{0} / \mathrm{PVA}$ membranes}

Data for the experiments using $\mathrm{Fe}^{0} / \mathrm{PVA}$ membranes are again presented as breakthrough curves, as shown in Figure 6 and summarized in Table 1. The data in Figures 6(a) through (e) for the pure PVA membranes, shown as solid points, have been adjusted with respect to $L$ and $C_{u p}$ so that the two curves can be directly compared. The data given in Table 1 for the pure PVA membranes are the uncorrected values. As before, each curve is characterized by its slope and intercept. The slope of the dashed lines, obtained by regression of data near the beginning of the breakthrough, should be equal to $\frac{C_{u p} P A}{L V_{\text {down }}}$ if the membrane is no longer reactive at this point.

Clear increases in the lag time are shown for carbon tetrachloride and $\mathrm{Cu}^{2+}$ in Figures 6(a) and (b). The lag times shown for $\mathrm{Fe}^{0} / \mathrm{PVA}$ membranes against carbon tetrachloride and $\mathrm{Cu}^{2+}$ are $353 \mathrm{~min}$ and $374 \mathrm{~min}$, respectively, while the corresponding lag times for pure PVA membranes are respectively $1.4 \mathrm{~min}$ and $1.0 \mathrm{~min}$ (after adjustments for $L$ and $C_{u p}$ ). The 

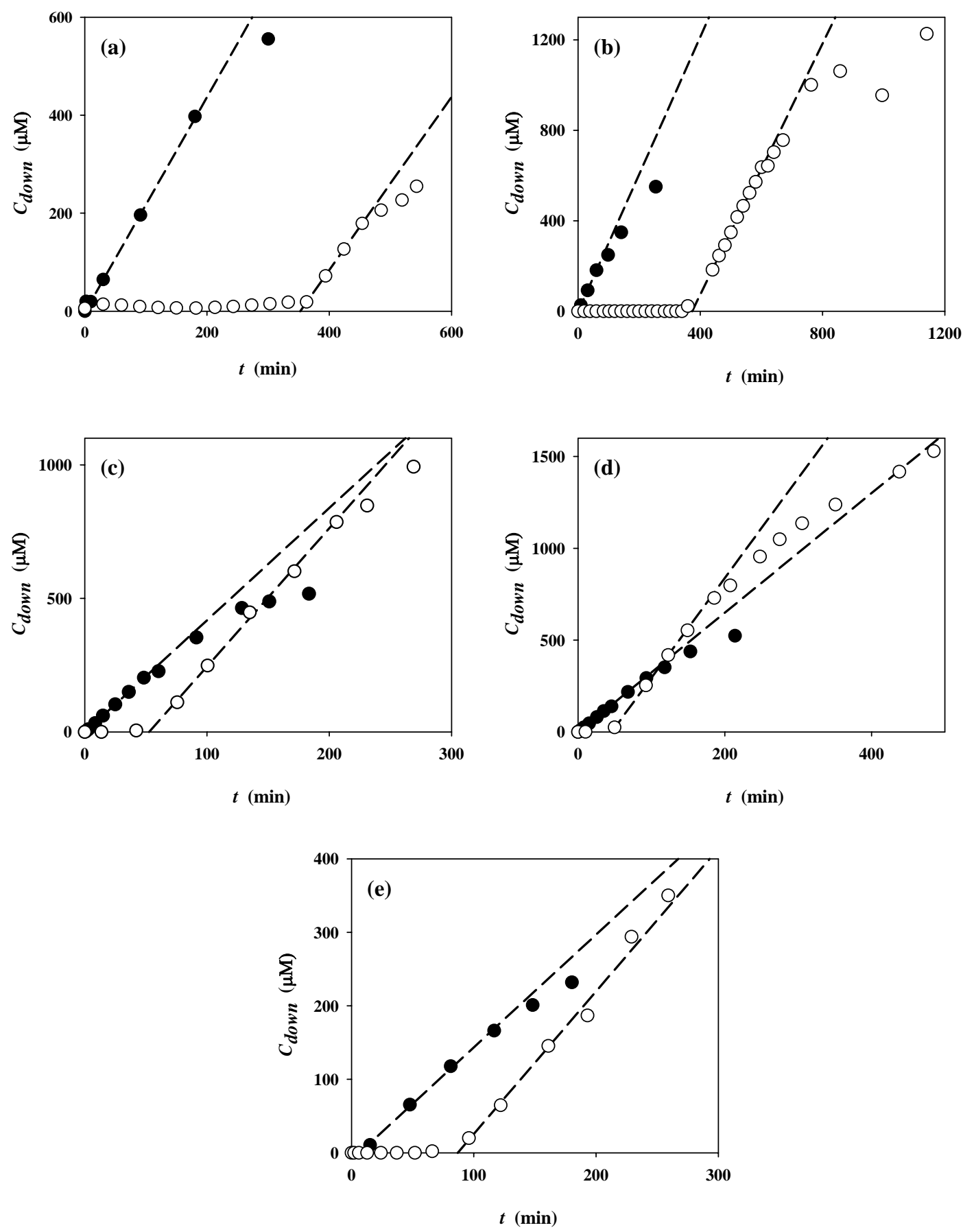

Figure 6. The breakthrough curves through $\mathrm{PVA}(\bullet)$ and $\mathrm{Fe}^{0} / \mathrm{PVA}(\mathrm{\circ})$ membranes: (a) carbon tetrachloride $\left(C_{u p}=4562 \mu \mathrm{M}, L=212 \mu \mathrm{m}, V_{\text {down }}=16.64 \mathrm{~mL}\right)$, (b) $\mathrm{Cu}^{2+}\left(C_{u p}=2995 \mu \mathrm{M}, L=102\right.$ $\left.\mu \mathrm{m}, V_{\text {down }}=13.11 \mathrm{~mL}\right)$, (c) nitrobenzene $\left(C_{u p}=2020 \mu \mathrm{M}, L=80 \mu \mathrm{m}, V_{\text {down }}=13.11 \mathrm{~mL}\right)$, (d) $4-$ nitroacetophenone $\left(C_{u p}=2424 \mu \mathrm{M}, L=89 \mu \mathrm{m}, V_{\text {down }}=13.11 \mathrm{~mL}\right)$, and $(\mathrm{e}) \mathrm{CrO}_{4}{ }^{2-}\left(C_{u p}=2512\right.$ $\mu \mathrm{M}, L=113 \mu \mathrm{m}, V_{\text {down }}=13.11 \mathrm{~mL}$ ). The dashed lines are related to the steady-state flux. Note the different concentration and time scales. 
Table 1. Summary of the breakthrough experiments.

\begin{tabular}{|c|c|c|c|c|c|c|c|c|c|}
\hline contaminant & No. & $\begin{array}{c}C_{0} \\
(\mathrm{M})^{\mathrm{a}}\end{array}$ & $\begin{array}{c}C_{u p} \\
(\mu \mathrm{M})\end{array}$ & $\begin{array}{l}V_{\text {down }} \\
(\mathrm{mL})\end{array}$ & $\begin{array}{c}L \\
(\mu \mathrm{m})\end{array}$ & $\begin{array}{c}P \\
\left(\times 10^{-9} \mathrm{~m}^{2} / \mathrm{min}\right)\end{array}$ & $\begin{array}{l}\text { observed } t_{\text {lag }} \\
\quad(\min )\end{array}$ & $\begin{array}{l}\text { predicted } t_{\text {lag }}{ }^{c} \\
(\min )\end{array}$ & $\begin{array}{c}\text { predicted } t_{\text {lag }} \\
\text { observed } t_{\text {lag }} \\
(-)\end{array}$ \\
\hline \multirow{5}{*}{$\mathrm{Cu}^{2+}$} & 1 & 0 & 5004 & 13.11 & 133 & $2.68^{\mathrm{d}}$ & $1.74^{\mathrm{d}}$ & - & $\frac{-}{1}$ \\
\hline & 2 & 0.44 & 5343 & 13.11 & 157 & 2.15 & 178.2 & 374 & 2.10 \\
\hline & 3 & 0.44 & 1018 & 13.11 & 200 & 2.68 & 1352.4 & 3186 & 2.36 \\
\hline & 4 & 0.44 & 2002 & 13.11 & 195 & 1.28 & 837.6 & 1540 & 1.84 \\
\hline & 5 & 1.25 & 2995 & 13.11 & 102 & 2.45 & 373.8 & 809 & 2.17 \\
\hline \multirow{3}{*}{ carbon tetrachloride } & 1 & 0 & 146 & 16.64 & 198 & $2.65^{b}$ & $1.04^{\mathrm{d}}$ & - & - \\
\hline & 2 & 1.24 & 4562 & 16.64 & 212 & 2.13 & 353 & 2305 & 6.53 \\
\hline & 3 & 1.24 & 3864 & 16.64 & 140 & 7.67 & 165 & 1187 & 7.19 \\
\hline \multirow{3}{*}{$\mathrm{CrO}_{4}^{2-}$} & 1 & 0 & 1598 & 13.11 & 295 & $1.79^{\mathrm{b}}$ & $45.4^{\mathrm{d}}$ & - & - \\
\hline & 2 & 0.44 & 5014 & 13.11 & 164 & 4.08 & 35.2 & 435 & 12.36 \\
\hline & 3 & 1.25 & 2512 & 13.11 & 113 & 2.26 & 86.9 & 1185 & 13.64 \\
\hline \multirow{3}{*}{ nitrobenzene } & 1 & 0 & 1669 & 13.11 & 88 & $4.31^{\mathrm{b}}$ & $0.54^{\mathrm{d}}$ & - & - \\
\hline & 2 & 1.25 & 3892 & 13.11 & 127 & 8.07 & 28.2 & 166 & 5.89 \\
\hline & 3 & 1.27 & 1877 & 13.11 & 80 & 5.30 & 52.6 & 155 & 2.95 \\
\hline \multirow{3}{*}{ 4-nitroacetophenone } & 1 & 0 & 1102 & 13.11 & 79 & $3.07^{b}$ & $0.51^{\mathrm{d}}$ & - & - \\
\hline & 2 & 0.41 & 2424 & 13.11 & 82 & 5.37 & 9.5 & 61.7 & 6.50 \\
\hline & 3 & 1.27 & 1997 & 13.11 & 89 & 6.27 & 45.9 & 272 & 5.92 \\
\hline
\end{tabular}

${ }^{\mathrm{a}}$ Iron concentration in the membrane is based on wet volume.

${ }^{\mathrm{b}}$ These permeability values for the pure, hydrated PVA membranes were used to calculate the predicted $t_{\text {lag }}$ for the $\mathrm{Fe}^{0} / \mathrm{PVA}$ membranes using eq 3 .

'The lag time was estimated using eq 3.

${ }^{\mathrm{d}}$ These values are not adjusted with respect to $L$ or $C_{u p}$ (see Appendix A). Adjusted values are given in the text. 
breakthrough for nitrobenzene shows a smaller increase caused by the iron incorporation. The breakthrough lag time for the $\mathrm{Fe}^{0} / \mathrm{PVA}$ membrane against nitrobenzene is $53 \mathrm{~min}$, while it is 0.45 min for the pure PVA membrane (again after adjustments). There are two obvious reasons for the smaller effect. First, nitrobenzene requires more $\mathrm{Fe}^{0}$ for reduction $(v=3)$ compared with carbon tetrachloride and $\mathrm{Cu}^{2+}(v=1)$. Second, the permeability of the membrane to nitrobenzene is higher than for the other two compounds, which also decreases the lag time (see eq 3 ).

The lag times in reactive membranes are predicted to be independent of reaction kinetics (Siegel and Cussler, 2003). To test this prediction 4-nitroacetophenone was selected as a model contaminant. 4-Nitroacetophenone reacts faster with $\mathrm{Fe}^{0}$ than nitrobenzene does (Devlin et al, 1998). As shown in Figure 6(d), the breakthrough lag time for 4-nitroacetophenone diffusing across the $\mathrm{Fe}^{0} / \mathrm{PVA}$ membrane is $46 \mathrm{~min}$, close to that for nitrobenzene through a similar $\mathrm{Fe}^{0} / \mathrm{PVA}$ membrane and thus consistent with the prediction. The lag time for 4nitroacetophenone is $0.65 \mathrm{~min}$ for the pure PVA membrane (after adjustments).

The breakthrough for $\mathrm{CrO}_{4}{ }^{2-}$ is affected less by the iron incorporation. The breakthrough lag time for the pure PVA membrane is 7 min (after adjustments), while it is $87 \mathrm{~min}$ for the $\mathrm{Fe}^{0} / \mathrm{PVA}$ membrane. Batch experiments show that $\mathrm{CrO}_{4}{ }^{2-}$ reacts very slowly when its concentration is high (e.g., $>1000 \mu \mathrm{M})$, probably because of deposition of $\mathrm{Cr}_{2} \mathrm{O}_{3}$ on the iron surface (Pratt et al., 1997; Melitas et al., 2001). The large lag time for the pure PVA membrane, which is reproducible, may reflect reaction of $\mathrm{CrO}_{4}{ }^{2-}$ with the PVA itself to form an ester (Duncalf and Dunn 1964; Van Nice and Farlee, 1977).

To investigate possible deposition of the heavy metals, we measured the chemical composition on the $\mathrm{Fe}^{0}$ /PVA membrane surface after exposure to the copper and chromate solutions. The spectra showed the presence of $\mathrm{Cu}$ and $\mathrm{Cr}$ on the membranes after reaction with $\mathrm{Cu}^{2+}$ and $\mathrm{CrO}_{4}{ }^{2-}$, respectively.

The permeability of the $\mathrm{Fe}^{0} / \mathrm{PVA}$ membranes to the selected contaminants ranged from 0.5 to 3 times that of the pure PVA. In general, the measured permeability of the reactive membranes was higher than that of the unmodified ones (Table 1). Ideally, the permeability of the membrane should not be affected by the incorporation of $\mathrm{Fe}^{0}$. Possible reasons for the observed deviation are discussed below.

To assess the significance of the reaction between $\mathrm{Fe}^{0}$ and water as a competing reaction during the contaminant breakthrough experiments, dissolved iron concentrations were measured in two diaphragm-cell experiments, one with carbon tetrachloride and the other without carbon tetrachloride. To complete an iron mass balance, the dissolution of iron during the hydration process prior to the experiments was also measured. The duration of hydration was 23-27 hours and the diaphragm-cell experiments were 9.75 hours. The iron mass balance is based on the exposed area in the diffusion experiment.

The results showed that $40-47 \%$ of the iron (based on the iron content in the dry membrane prior to hydration) dissolved during the hydration process, while the dissolution of the iron during the diffusion experiment was much smaller (only $5 \%$ relative to the hydrationrelated loss). No difference in iron dissolution was observed between the two diaphragm cell experiments (with and without carbon tetrachloride).

\section{Discussion}

The results above show that the breakthrough lag time of polymer membranes used as contaminant barriers can potentially be dramatically increased by incorporation of reactive 
groups or materials within the polymer material. Although the choice of $\mathrm{Fe}^{0} / \mathrm{PVA}$ system was designed to permit experiments to be conducted over short time scales, we expect that improvements in the barrier properties of PVA can be easily secured for other polymers such as polyethylene. This expectation has considerable experimental support from this work, and many other reagent choices are possible.

The results with the $\mathrm{Fe}^{0}$ /PVA membranes show substantial increases in the lag time before there is significant permeation through the barriers. For copper, the lag is increased 100 to 400 fold; for carbon tetrachloride, it rises about 300 fold; for the nitroaromatics, 20 to 100 fold. The lag time increased only 13 fold for chromate, a solute for which little reaction is expected. These numbers are adjusted for $L$. Once the membrane is broached, the leak rate is regulated by a permeability which typically changes by less than a factor of two, but up to a factor of three (based on the slope of the breakthrough curve) from a non-reactive membrane.

The observed changes in permeability merit further discussion, as the permeability influences further analysis of the lag time data. When hydrated, PVA is 50\% water (pore space), leading to its high permeability. For a membrane containing $1.25 \mathrm{M}$ iron, calculations reveal that the void volume would increase approximately $1 \%$ upon complete dissolution of the iron nanoparticles. Thus, changes in permeability of the membrane due to $\mathrm{Fe}^{0}$ dissolution should be minimal. The larger changes in permeability observed suggest that other changes in the membrane chemistry and geometry are occurring. The dissolution of the iron could lead to a shunt forming through the membrane by connecting pores that already exist in the PVA matrix. As shown in Figure 2, the iron is not completely dispersed in the membrane and forms 1-2 $\mu \mathrm{m}$ clusters of smaller nanoparticles. Their dissolution can be envisioned to provide a shortcut across a 100-200 $\mu \mathrm{m}$ barrier, which leads to the higher permeability. Such pore connectivity is much less likely in polymers (i.e. HDPE or PVC) used for practical barriers. When developing these practical membranes, however, observed changes in permeability for the reactive membranes will be an important piece of information in determining optimum $\mathrm{Fe}^{0}$ loadings to prevent shunt formation, and thus averting poor membrane performance after contaminant breakthrough.

Because permeability provides a reference value for lag time calculations (eq 3 ), subsequent analyses assume that the appropriate values of $P$ to use in the interpretation of the lag time results are those measured for the pure PVA membranes. For each compound, multiple experiments with pure PVA films were run. The selected $P$ for each contaminant is the lowest, reproducible value measured for the PVA membranes, which we deem to be from membranes with the fewest possible number of defects. These values are those reported in Table 1.

Despite the variations in permeability, our results demonstrate that the performance (i.e. lag time) of a contaminant barrier can be dramatically improved by chemical reaction. A raw extrapolation of our results would predict that a polymer barrier membrane could ideally be made effective more than 100 times longer with the inclusion of reactive material. This improved effectiveness, however, is not guaranteed. First, it is necessary to identify a particular chemistry which can consume the contaminant sufficiently rapidly. Second, the ratio of the reaction rate to the diffusion rate must be large, i.e. the second Damköhler number must be much greater than one. Third, any reactive material must be accessible and must be completely consumed for the lag time to increase by the largest possible extent.

These assumptions are tested using eq 3 to predict the diffusion lag time for the $\mathrm{Fe}^{0} / \mathrm{PVA}$ membranes. The required parameters $\left(L, C_{0}, C_{u p}, v, P\right)$ are all known from the experiments, where $P$ is obtained from a diffusion experiment using a pure PVA membrane for the reasons 
described above. Table 1 compares predicted lag times with experimental lag times. In all cases, predicted lag times are larger. The ratio of the predicted to experimental values is smallest for

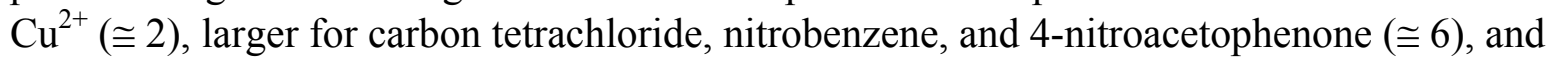
largest for $\mathrm{CrO}_{4}{ }^{2-}(\cong 13)$. This means that $\mathrm{Cu}^{2+}$ obeys eq 3 much better than $\mathrm{CrO}_{4}{ }^{2-}$.

This discrepancy is probably due to the fact that not all of the iron in the membrane was consumed by reaction with contaminants by the time of breakthrough. To determine how much of the iron is actually available for the reaction, $C_{0}$ in eq 3 is replaced by an "effective iron concentration", $C_{0}^{\text {eff }}$ :

$$
t_{\text {lag }}=\frac{L^{2} C_{0}^{\text {eff }}}{2 v P C_{u p}}=\frac{L^{2} C_{0}}{2 v P C_{u p}} \frac{C_{0}^{e f f}}{C_{0}}
$$

A plot of $t_{\text {lag }}$ against $\frac{L^{2} C_{0}}{2 v P C_{u p}}$ for each contaminant should give a straight line with the slope of $\frac{C_{0}^{e f f}}{C_{0}}$, a fraction of iron accessible for the particular compound. Figure 7 shows

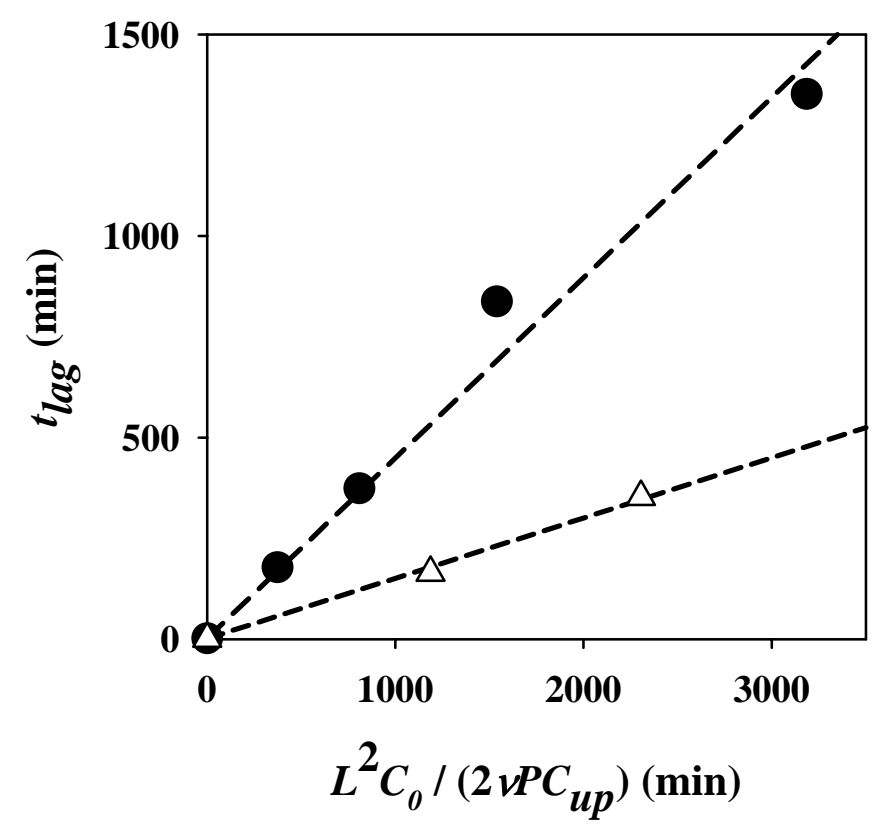

Figure 7. Dependence of the breakthrough lag time on the aggregated parameter $C_{0} L^{2} /\left(2 v P C_{u p}\right)$ for carbon tetrachloride $(\Delta)$ and $\mathrm{Cu}^{2+}(\bullet)$. The dashed lines are linear regressions with their $\mathrm{y}-$ intercept fixed at the lag time for the pure PVA membrane $\left(\mathrm{R}^{2}=0.996\right.$ for $\Delta$ and 0.977 for $\left.\bullet\right)$.

such a plot applied to $\mathrm{Cu}^{2+}$ and carbon tetrachloride. The slope of the line is $0.45 \mathrm{for}^{2+}$ and 0.15 for carbon tetrachloride: only $45 \%$ of the iron (based on the iron content in the dry membrane prior to hydration) was consumed by reaction with $\mathrm{Cu}^{2+}$ and $15 \%$ by reaction with carbon tetrachloride. The same analysis shows that $25 \%, 17 \%$, and $6 \%$ of the iron was consumed by nitrobenzene, 4-nitroacetophenone, and $\mathrm{CrO}_{4}{ }^{2-}$, respectively. When accounting for 
the $40-47 \%$ of the iron lost during hydration of the membrane prior to conducting the breakthrough experiments, these percentages increase by approximately a factor of two. Considering these numbers, most of the iron in the membrane (about $90 \%$ ) was "consumed" before the breakthrough of $\mathrm{Cu}^{2+}$ occurs. For the other compounds, $50-70 \%$ of the iron was "consumed" during the hydration and reaction periods.

A large portion of the iron (> $30 \%$ ), however, is still unaccounted. Other possibilities for the difference between predicted and observed lag times include: oxidation of $\mathrm{Fe}^{0}$ by reaction with water during the breakthrough experiment and formation of a surface reaction product film which reduces the reaction rate.

The presence of carbon tetrachloride during the diffusion experiment did not significantly increase the measured concentrations of dissolved iron. This implies that the iron oxidized by contaminants is not released into the solution but remains in the membrane as a solid oxide phase. This idea is supported by an SEM image of the $\mathrm{Fe}^{0} / \mathrm{PVA}$ membrane after the experiment in which the nanoparticles can be still seen. Visual inspection of the membrane after exposure to carbon tetrachloride suggests that at least a portion of the iron remains in the membrane as an iron oxide, for the exposed area of the membrane is an orange/brown color common to iron oxide solids. It is unlikely, however, that all of the "remaining iron" in the membrane is oxidized. The formation of an oxide layer will slow the reaction of oxidized contaminants with $\mathrm{Fe}^{0}$, (Scherer et al., 1997; Farrell et al., 2000) likely to a point where diffusion through the membrane becomes faster than reaction (i.e. the Damköhler number becomes small), and contaminant breakthrough occurs. Thus, we suspect that a large portion of the $\mathrm{Fe}^{0}$ is covered by iron oxide and unavailable to the contaminants (carbon tetrachloride, nitrobenzene, and 4nitroacetophenone) diffusing through the membrane. In the case of $\mathrm{CrO}_{4}{ }^{2-}$, the $\mathrm{Fe}^{0}$ surface is likely covered by precipitated $\mathrm{Cr}_{2} \mathrm{O}_{3}$ as mentioned above, resulting in a similar effect.

One potential strategy to make a larger fraction of the iron accessible would be to use smaller iron particles. This method, however, has two disadvantages. The larger specific surface area could lead to faster reaction not only with contaminants but also with water. The difficulty and potential cost associated with synthesis of smaller iron particles may be prohibitive as well. 


\section{Permeable Membranes Containing Crystalline Silicotitanate As Model Barriers For Cesium Ion}

This portion of the work tested a permeable barrier membrane for cesium ion. These experiments serve as the first step towards impermeable membranes which could be used to reduce the risk of leaks of radioactive cesium waste. The development has three parts: the adsorption isotherms of the ion exchangers used, chloride breakthrough as a test system, and cesium breakthrough using the permeable model system. In the paragraphs below, these three parts are described sequentially, with the most detail for the cesium barrier.

In these experiments, the reaction chemistry is altered from that of an irreversible transformation to an ion exchange process. This requires a modification of the theory presented above. If the reaction is reversible with a linear isotherm,

$$
t_{\text {lag }}=\frac{L^{2}}{6 D}(1+K)
$$

where $K$ is the equilibrium constant for the isotherm (Paul and Koros, 1976). If the reaction is reversible with a saturated Langmuir isotherm, the result is known analytically but is complicated (Paul and Koros, 1976). As shown below, however, our experiments are in the limit where the isotherm is almost flat. In other words, the reagents in the membrane quickly become saturated with cesium, and this adsorbed and immobilized cesium concentration is dramatically larger than the concentration of non-adsorbed cesium. In this limit, the complicated analytical expressions for $t_{\text {lag }}$ simplify dramatically to give

$$
t_{\text {lag }}=\left(\frac{L^{2}}{2 D H}\right) \frac{c_{20}}{c_{10}}
$$

where $c_{20}$ is now the total number of adsorbing sites on species 2, the CST; and $c_{10}$ is again the upstream cesium concentration. This result is mathematically equivalent to that for an irreversible reaction given in Equation 3, although its physical basis is different. We expect any favorable isotherm will give the same result if the reaction is sufficiently rapid. These equations are used in analyzing the experimental results that follow.

\section{Isotherm}

The adsorption isotherm for cesium ion is shown in Figure 8. The cesium/CST data show strong adsorption reaching saturation at about $10^{-4} \mathrm{M}$ solution concentration. Although a saturation value exists, the data at low concentrations do not fit a Langmuir isotherm but the data set do approximate a Freundlich isotherm, as illustrated in the inset of this figure. The Freundlich isotherm also better approximates the observed shoulder than does a Langmuir isotherm. The best fit of these data is

$$
c_{2}^{*}=0.0018 c_{1}^{0.0962}
$$


where $c_{2}^{*}$ is the cesium concentration in the CST in moles per gram, and $c_{1}$ is the molar concentration of cesium in water at equilibrium. The apparent saturation value, $q_{\max }$, for CST was found to be $0.9 \frac{\mathrm{mmol}}{\mathrm{gram}}$ for $\mathrm{Cs}^{+}$. This value is larger than the value reported by Gu et al.

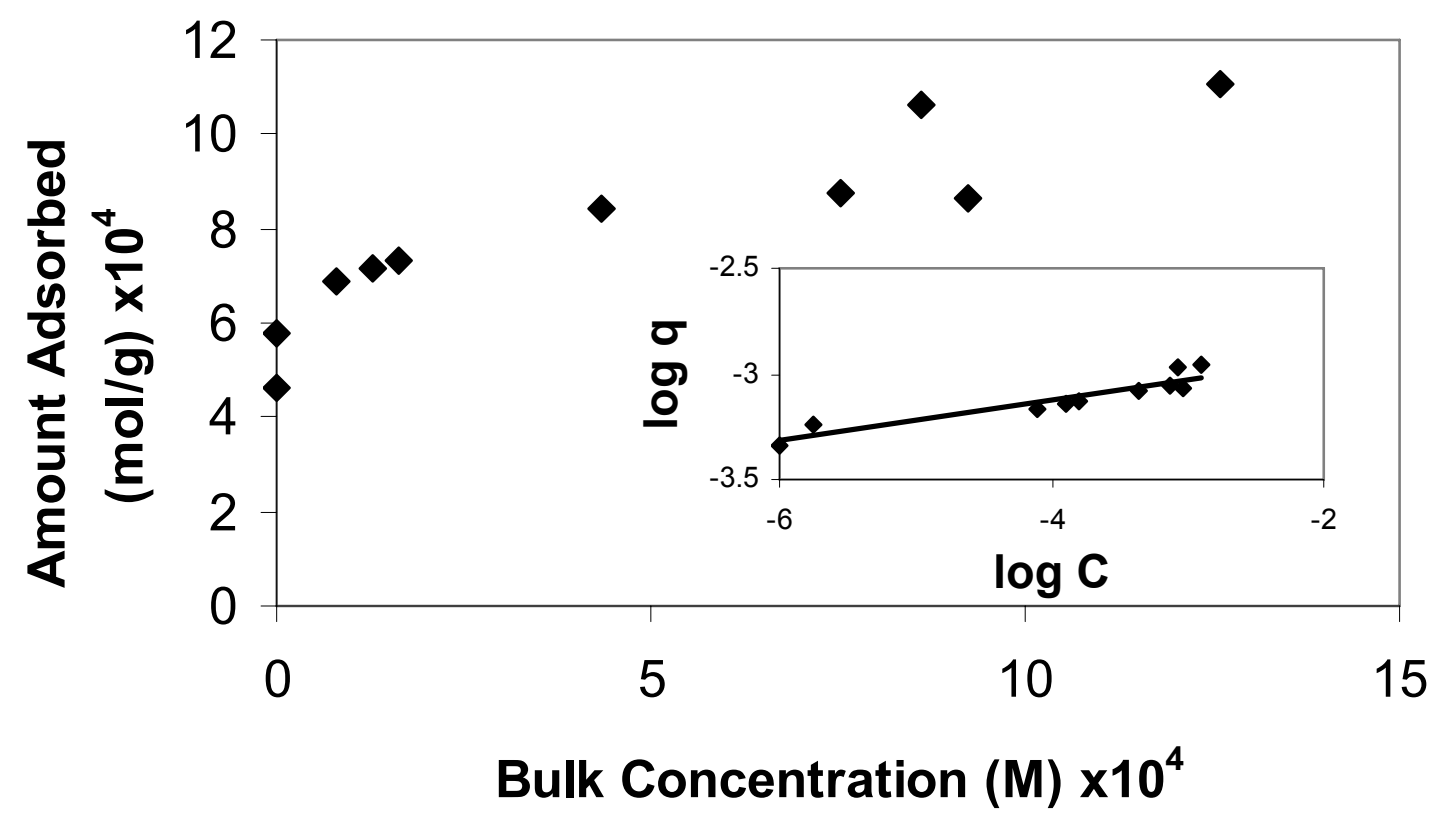

Figure 8: Isotherm for the cesium/CST system. (Inset) This system can be approximated by a favorable Freundlich isotherm.

(1997) and Zheng et al. (1996) of $0.56 \frac{\mathrm{mmol}}{\mathrm{gram}}$. One possible reason for this difference is that in these previous studies the measurement was made in a solution containing 5.1 $\mathrm{M} \mathrm{NaNO}_{3}$, while our measurement was made in aqueous cesium nitrate alone.

\section{Breakthrough Experiments}

For membranes containing CST, the effects of upstream $\mathrm{Cs}^{+}$concentration, barrier thickness, and CST concentration were measured. The results for different upstream concentrations at a constant CST loading are given in Figure 9(a). Decreasing the feed concentration increases the lag as Equations 3 and 5 predict. (Because the isotherm is not linear, Equation 4 is not applicable.) Note that these equations imply that the slopes after breakthrough should be the same. Those for 0.1 and $0.001 \mathrm{M}$ are, but that for $0.01 \mathrm{M}$ is smaller.

The effect of different membrane thickness is shown in Figure 9(b). As expected, the lag times increase sharply for thicker films. The slopes after breakthrough are smaller for the thicker membranes as expected. The effect of differing CST concentrations is illustrated in Figure 9(c). Increases are dramatic, with lag times increasing by a factor of six when the CST concentration increases by a factor of four. This is more than the factor of four expected from Equations 3 and 5 , which predict a linear variation with CST concentration. The slope of concentration versus 
time after breakthrough, expected to be constant, drops about four times over the loading range studied.

We were concerned that the lags and the concentration variations showed effects different than the theory. On the one hand, we are pleased that the lags are bigger than expected and that the leaks occur more slowly than expected. Thus the barriers seem even better than anticipated.
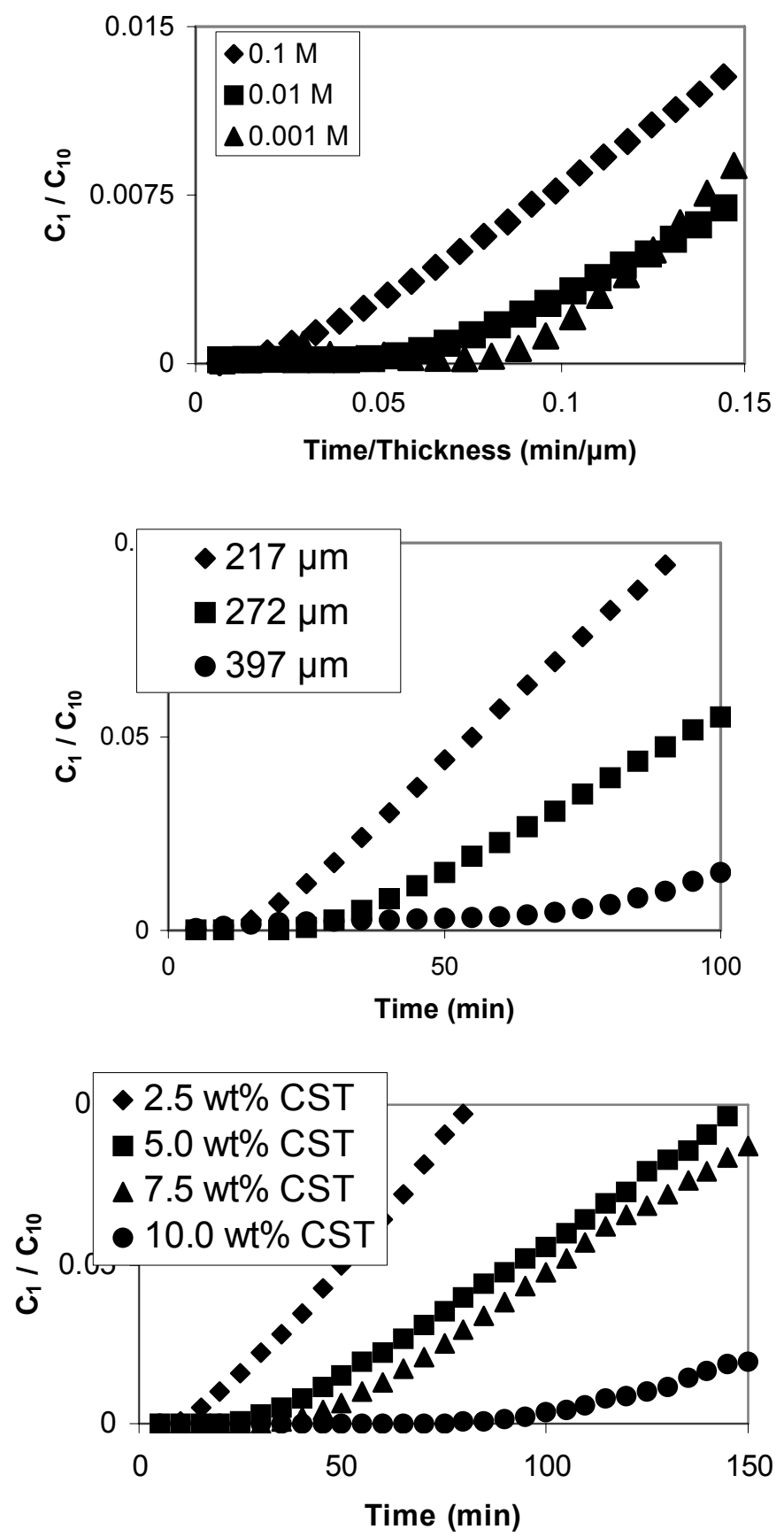

Figure 9. Effects of upstream concentration (top), membrane thickness (middle), and CST loading on barrier performance. 
On the other hand, the theory summarized above has been widely verified, both by us and by others (Yang et al., 2001; Bansleben et al., 2001; Schwank et al., 2004). Some uncertainty in our measurements is suspected.

We have identified two sources of this uncertainty. First, it is assumed that the upstream concentration is constant during the experiment, as implied by Equation 1. For nonreactive membranes, it changes less than 3 percent. For some reactive membranes studied, this concentration has dropped significantly. If a CST laden membrane is used, the breakthrough curve shows a long lag and a small slope. The long lag is expected because of cesium adsorption by the CST. If an additional breakthrough experiment is performed using the same, exhausted membrane, the breakthrough curve shows almost no lag and a larger slope. The small lag is expected because the CST is now largely saturated with cesium from the first run. The larger slope is believed to result because the upstream concentration is not depleted as much by reaction, and so the flux stays high. Thus a depleted upstream concentration may be part of the reason for the smaller slopes at higher CST concentrations shown in Figure 9(c).

The second source of uncertainty comes from the location of the CST in the membrane. Experiments and theories utilizing detailed numerical models which are more complete than those summarized above show that where the reaction occurs matters (Nuxoll et al., 2005). If the reaction occurs on the upstream membrane face, the lag is half that when the reaction occurs on the downstream face. If the CST settles while the membrane is being made, the lag time will be different depending on the orientation of the membrane.

To test whether membrane orientation was important, experiments were made in which we deliberately changed membrane orientation. Normally, the experiments used membranes with the top surface of the membrane during fabrication oriented towards the more concentrated, "upstream" volume. This meant that the downward face, which was oriented towards the "downstream" volume, may have a higher CST concentration than the membrane's average because of CST settling during fabrication. Changing the membrane's orientation does change the lag time about 30 percent, suggesting that the CST distribution within the membrane is not completely uniform (see Supporting Information). This second uncertainty is still much less than the increased lag of 3000 percent between the original membranes and those with added CST.

\section{Discussion}

As shown above, the results obtained here are roughly consistent with the theoretical predictions. Adding CST to a barrier membrane, making the membrane thicker, and reducing the upstream cesium concentration all delay the penetration of cesium across the barrier membranes. This delayed penetration, summarized as a lag time, can be increased by more than a factor of thirty. This large effect makes these membranes interesting.

The increases in lag times observed in our experiments roughly agree with those predicted, as shown in Figure 10. In fact, the measured lags seem somewhat longer than those predicted; i.e., our actual results are better than expected. As discussed above, we believe that part of the reason for this discrepancy may be the nonuniform distribution of CST within the membrane.

There is a second, more fundamental reason, however, why the membranes are behaving better than expected. This is the result of the nonlinear isotherm operating in this 


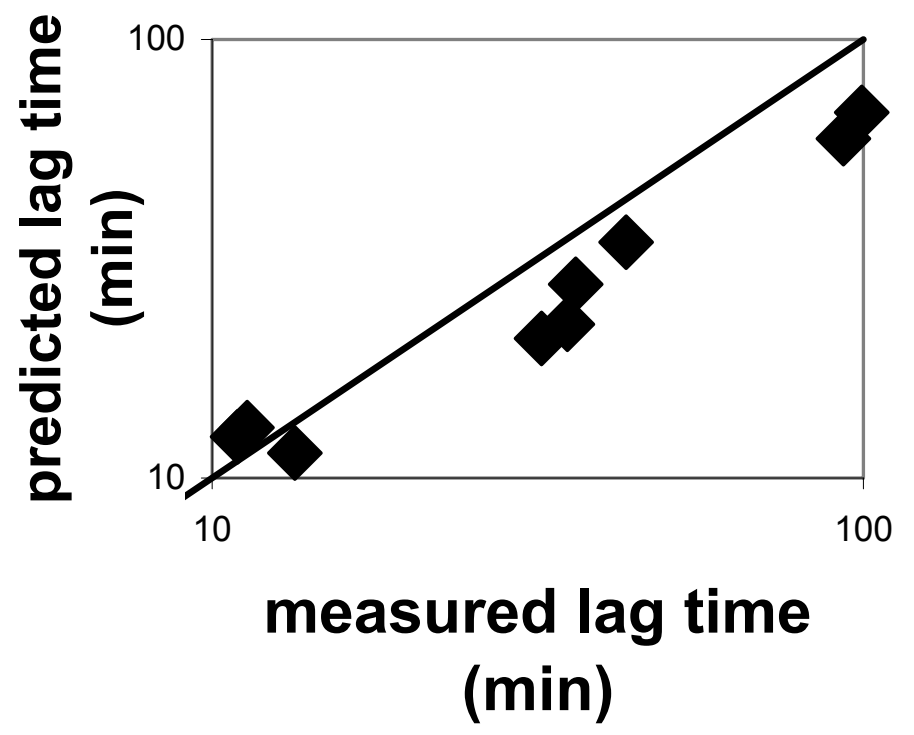

Figure 10: Measured lag times correlate with theoretical predictions.

system and shown in Figure 8. To explore the effect of this nonlinear isotherm, we imagine first that the reaction between cesium and CST is instantaneous and irreversible, and that it occurs at some position $\ell^{\prime}$ within the membranes. The rate of this reaction is then diffusion controlled

$$
j_{1}=\frac{D H}{\ell^{\prime}}\left(c_{10}-0\right)
$$

where $j_{l}$ is the diffusive flux of cesium and $\ell^{\prime}$ is the reaction location. The reaction location changes with time according to the mass balance

$$
c_{20} \frac{d \ell^{\prime}}{d t}=\frac{D H}{\ell^{\prime}}\left(c_{10}-0\right)
$$

Because the reaction starts at the upstream surface of the membrane, $\ell^{\prime}$ is zero when $t$ is zero, and Equation 8 is easily integrated to give

$$
\ell^{\prime}=\sqrt{2 D H c_{10} t / c_{20}}
$$

When the reaction location has crossed the membrane, $\ell^{\prime}$ equals the membrane thickness $L$ and

$$
t_{\text {lag }}=\left(\frac{L^{2}}{2 v D H}\right) \frac{c_{20}}{c_{10}}
$$

which is identical to Equation 2.

Now we imagine that the reaction is instantaneous but stops at some equilibrium concentration $c_{i e}$, which is roughly the shoulder of the isotherm shown in Figure 8. This shoulder is an approximation, most accurate for Freundlich isotherms with an exponent much 
less than one. The exponent in Equation 6 is about 0.10. In this case, Equation 7 should be replaced by

$$
j_{1}=\frac{D H}{\ell^{\prime}}\left(c_{10}-c_{i e}\right)
$$

which leads to

$$
t_{\text {lag }}=L^{2} c_{20} / 2 D H\left(c_{10}-c_{i e}\right)
$$

This does predict that the lag times should be slightly longer than those expected, and hence is consistent with the data in Figure 10. At this point, however, we are unsure if this argument is more significant than a nonuniform distribution of CST in our experiments. 


\section{Model membrane performance using Hanford Groundwater}

In this section, we replace the Tris buffer solution (used in Fe(0)/PVA experiments) and deionized water (used in CST/PVA experiments) with uncontaminated groundwater collected from the Hanford site. The goal is to determine any matrix effects on membrane/reactive material performance. Both breakthrough experiments described previously and batch experiments in which the membrane was placed in a sealed serum bottle filled with the media of interest were used.

\section{Results}

Breakthrough experiments with pure PVA membranes using Tris buffer and Hanford water are compared in Table 2 and Figure 11. Using regressions of the linear portions of curves shown in Figure 11 and those not pictured (for better visualization), the permeabilities of the PVA membranes were $(8.1 \pm 0.6) \times 10^{-11} \mathrm{~m}^{2} / \mathrm{s}$ and $(7 \pm 1) \times 10^{-11} \mathrm{~m}^{2} / \mathrm{s}$ in Tris buffer and in Hanford water, respectively, using carbon tetrachloride (CT) as a model contaminant.

Table 2. Summary of Breakthrough Experiments with Pure PVA and CT as the Contaminant

\begin{tabular}{|c|c|c|c|c|c|c|}
\hline & $\begin{array}{c}C_{u p} \\
(\mu \mathrm{M})\end{array}$ & $\begin{array}{c}L \\
(\mathrm{~mm})\end{array}$ & $\begin{array}{c}P \\
\left(\times 10^{-11} \mathrm{~m}^{2} / \mathrm{s}\right)\end{array}$ & $\begin{array}{c}t_{\text {lag }} \\
(\mathrm{min})\end{array}$ & $\begin{array}{c}D \\
\left(\times 10^{-11} \mathrm{~m}^{2} / \mathrm{s}\right)\end{array}$ & $H$ \\
\hline Buffer 1 & 1000 & 0.066 & 8 & 0.3 & 5 & 2 \\
\hline Buffer 2 & 2196 & 0.080 & 10 & 0.05 & 38 & 0.3 \\
\hline Buffer 3 & 3960 & 0.120 & 11 & 0.7 & 6 & 2 \\
\hline Buffer 4 & 1229 & 0.25 & 7.1 & 0.3 & 58 & 0.1 \\
\hline Buffer 5 & 2245 & 0.250 & 7.2 & 0.7 & 26 & 0.3 \\
\hline Buffer 6 & 1739 & 0.491 & 6 & 0.8 & 85 & 0.1 \\
\hline Hanford Water & 1826 & 0.42 & 7 & 9 & 5 & 1.4 \\
\hline
\end{tabular}

Figure 12 is used to compare the ability of the reactive $\mathrm{Fe}^{0}$ to extend the lag time of the membrane barrier. The basis for this comparison is outlined in Equations 13 and 14, which are a mass balance of CT in the liquid media surrounding the membranes in a batch reactor and the definition of the lag time for a reactive membrane. In Equation 13, the surface area $A$ is defined as the entire surface area of the membrane (i.e. both sides), because diffusion occurs equally into both faces in the batch experiments. This is different than the way $A$ is defined for breakthrough experiments. In Equations 13 through 15, $V$ is the volume of the aqueous media surrounding the membrane in a batch reactor, $C_{1}$ is the concentration of CT in the aqueous media, $C_{10}$ is the initial CT concentration, and $C_{0}$ is the initial concentration of $\mathrm{Fe}^{0}$ in the hydrated PVA membrane.

$$
\begin{aligned}
& V \cdot \frac{d C_{1}}{d t}=\frac{A \cdot P \cdot C_{1}}{L} \\
& t_{\text {lag }}=\frac{L^{2} \cdot C_{0}}{2 \cdot v \cdot P \cdot C_{1}}
\end{aligned}
$$




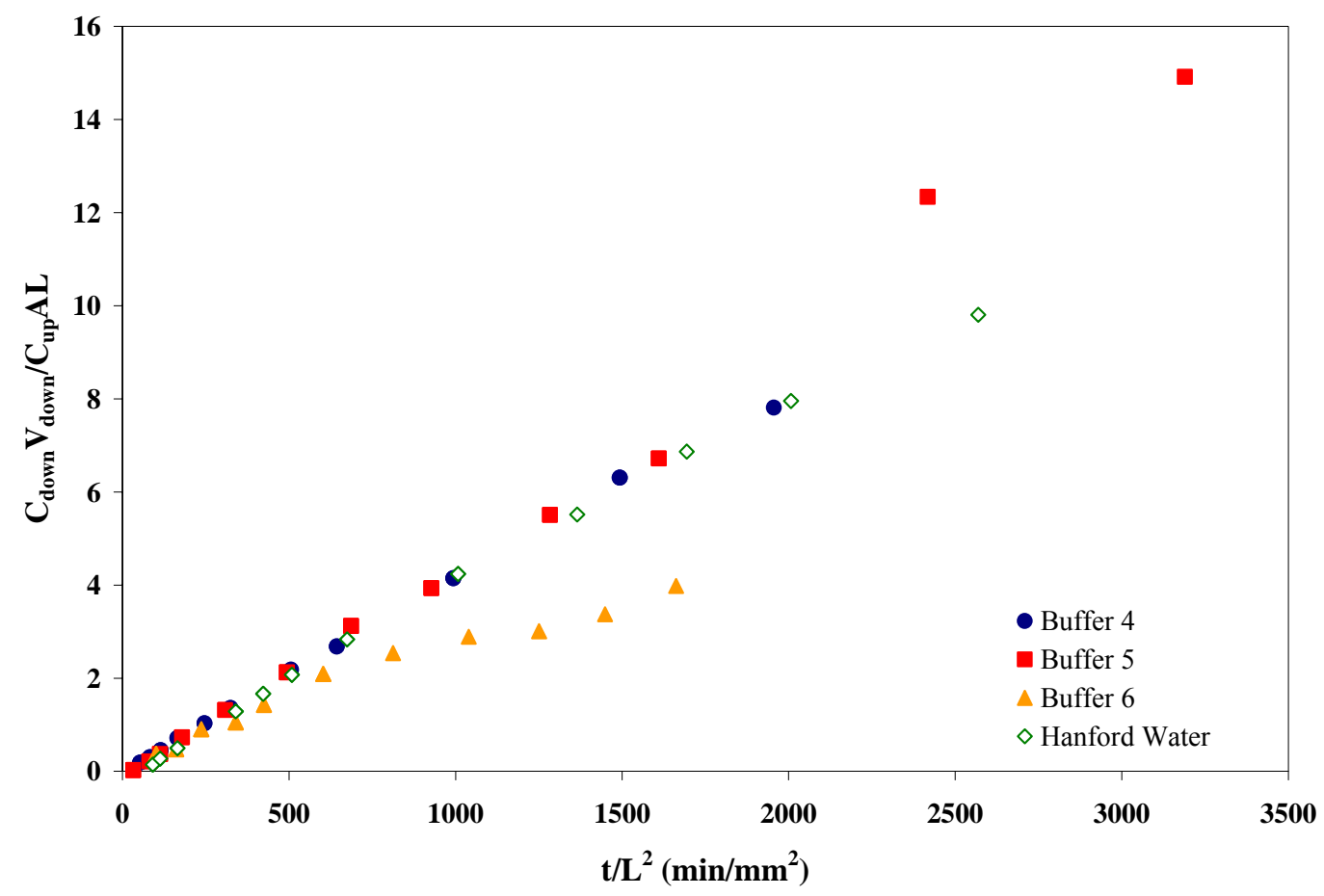

Figure 11. Comparison of the flux of carbon tetrachloride through pure PVA membranes when the liquid media surrounding the membranes is either a $50 \mathrm{mM}$ Tris buffer solution or groundwater from the DOE's Hanford site. Data from all six Tris buffer experiments and the Hanford water experiment are included in Table 1.

When combined, these equations yield an expression that describes the depletion of CT in the aqueous phase as a function of time. This equation is valid during an initial period when the assumption that $H C_{1}-C_{\text {center of membrane }} \approx H C_{1}$, or the lag time portion of a breakthrough-type experiment. The initial portions of experiments displayed in Figure 12 follow this linear trend.

$$
C_{1}^{1 / 2}=C_{10}^{1 / 2}-\frac{A}{V}\left(\frac{P \cdot C_{2, \text { ffective }} \cdot t}{2}\right)^{1 / 2}
$$

Table 3 contains data from both series of experiments. When $P$ determined from the pure PVA breakthrough experiments is applied, it is possible to determine an "effective" concentration of $\mathrm{Fe}^{0}$ in the membrane by solving Equation 15 for $C_{2, \text { effective. }}$. For experiments where Tris buffer was maintained at a $\mathrm{pH}$ of 7.2, the iron usage, or $C_{2, \text { effective }}$ divided by the actual iron concentration in the hydrated membrane, was approximately 2 times higher in the buffered reactors than in the Hanford water. When the Tris buffer $\mathrm{pH}$ was adjusted to match the $\mathrm{pH}$ of the Hanford water (buffer $\mathrm{pH}=8.8$ ), the membranes performed even better, with an effective iron usage nearly 2.7 times that of the Hanford water, though this latter difference was not statistically significant. 


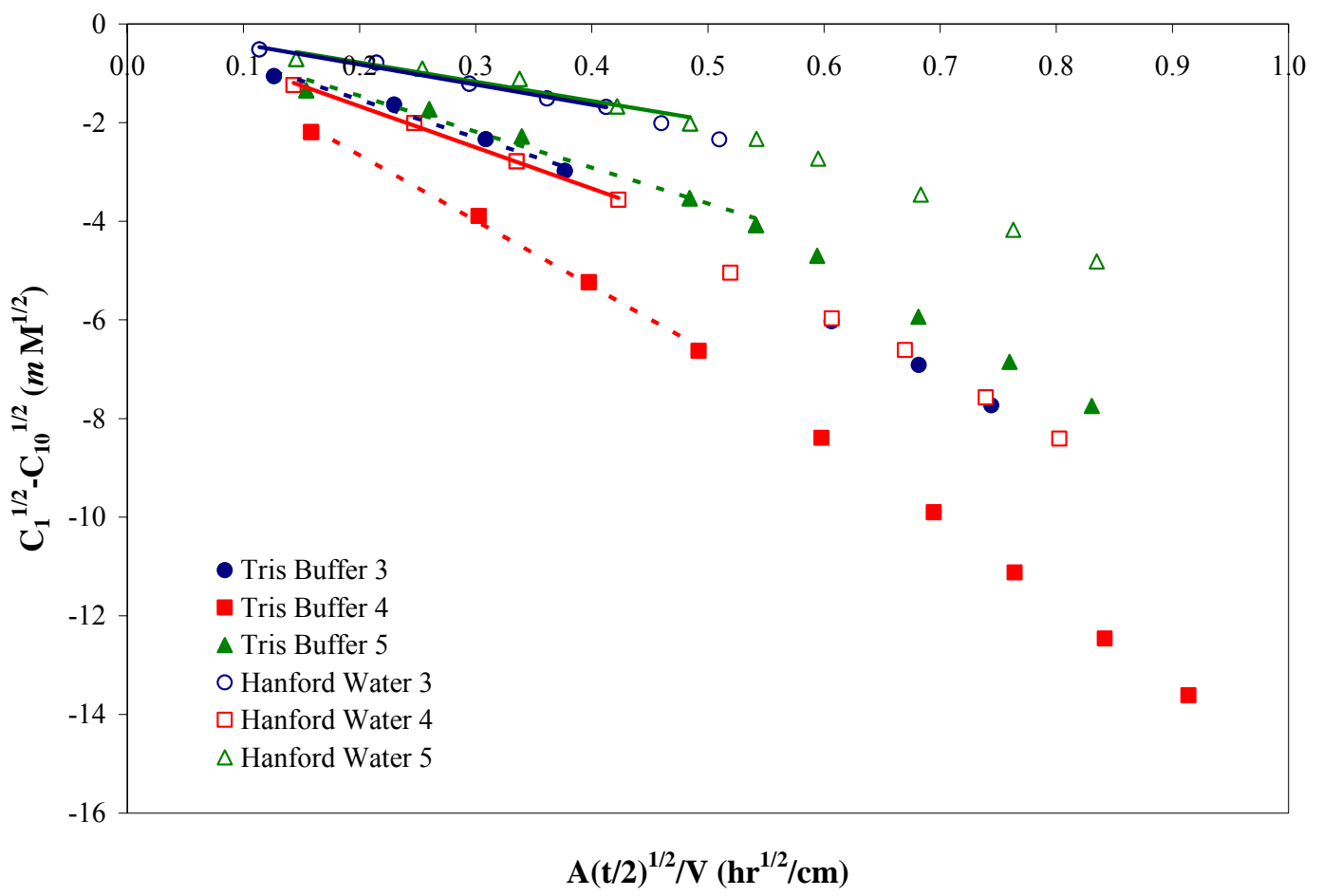

Figure 12. Comparison of the depletion of CT by PVA membranes containing $\mathrm{Fe}^{0}$ nanoparticles in batch reactors with liquid media surrounding the membranes as either a $50 \mathrm{mM}$ Tris buffer solution $(\mathrm{pH}=8.8)$ or groundwater from the DOE's Hanford site. Lines represent regressions of the initial linear period represented by Equation 3. For experimental variables, see Table 2.

Table 3. Summary of Batch Experiments with CT and PVA Membranes Containing $\mathrm{Fe}^{0}$

\begin{tabular}{|l|c|c|c|c|c|c|c|c|c|c|}
\hline & \multicolumn{9}{|c|}{ Tris Buffer } & \multicolumn{5}{c|}{ Hanford Water } \\
\cline { 2 - 11 } & $\mathrm{pH}$ & $\begin{array}{c}\mathrm{C}_{10} \\
(\mu \mathrm{M})\end{array}$ & $\begin{array}{c}\mathrm{C}_{2} \\
(\mathrm{M})\end{array}$ & $\begin{array}{c}\mathrm{C}_{2, \text { effective }} \\
(\mathrm{M})\end{array}$ & $\% \mathrm{Fe}^{0}$ usage & $\mathrm{pH}$ & $\begin{array}{c}\mathrm{C}_{10} \\
(\mu \mathrm{M})\end{array}$ & $\begin{array}{c}\mathrm{C}_{2} \\
(\mathrm{M})\end{array}$ & $\begin{array}{c}\mathrm{C}_{2, \text { effective }} \\
(\mathrm{M})\end{array}$ & $\% \mathrm{Fe}^{0}$ usage \\
\hline 1 & 7.2 & 210 & 1.2 & 0.015 & 1.3 & 8.8 & 340 & 1.2 & 0.006 & 0.5 \\
\hline 2 & 7.3 & 180 & 1.3 & 0.029 & 2.3 & 9.3 & 170 & 1.3 & 0.017 & 1.3 \\
\hline 3 & 8.8 & 360 & 1.6 & 0.060 & 4 & 8.7 & 320 & 1.6 & 0.026 & 1.6 \\
\hline 4 & 8.8 & 190 & 1.0 & 0.020 & 2 & 8.7 & 180 & 1.0 & 0.006 & 0.6 \\
\hline 5 & 8.8 & 200 & 1.1 & 0.018 & 2 & 8.7 & 190 & 1.1 & 0.006 & 0.5 \\
\hline
\end{tabular}

For membranes containing CST, an potentially important factor is the ionic strength of the water compared to the distilled water. The Hanford water has an ionic strength of about 0.013 $\mathrm{M}$, largely because of calcium and magnesium carbonates and sulfates, and a $\mathrm{pH}$ of 8.18. In these experiments samples of Hanford water were spiked with $0.001 \mathrm{M} \mathrm{CsNO}_{3}$ and then placed in the upstream compartment of our cell. Typical results, shown in Figure 13, show lag times across membranes with five weight percent CST which are as much as 40 times larger than across membranes without CST. Again, adding CST improves the membrane's barrier properties. In fact, the increase with the Hanford water is about 3 times larger than in distilled water. 


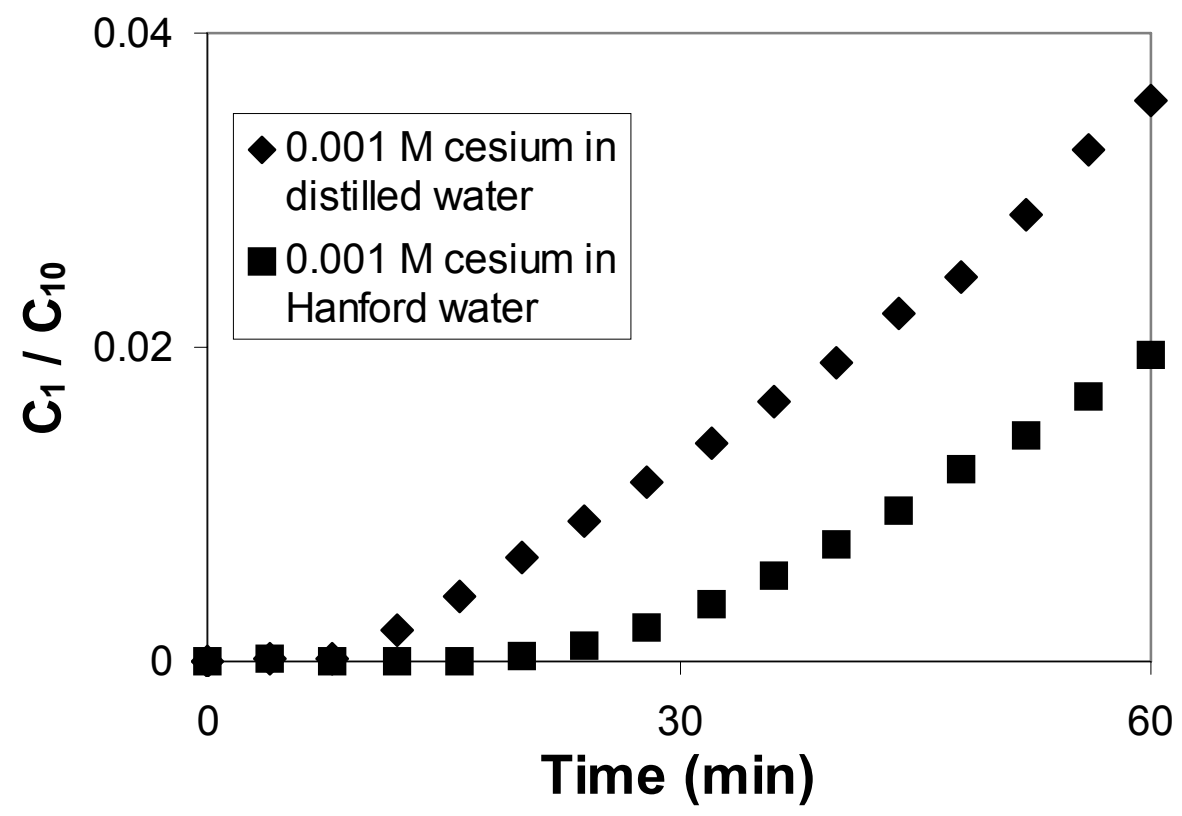

Figure 13: Measurement of breakthrough for cesium added to groundwater from Hanford, WA shows increased lag time when diffusing through a $5.0 \mathrm{wt} \% \mathrm{CST}$ membrane. The lag time is nearly three times longer for the Hanford water trial $(197 \mu \mathrm{m} ; 28.3 \mathrm{~min}$.) compared to that for distilled water $(195 \mu \mathrm{m} ; 9.6 \mathrm{~min}$.).

\section{Discussion}

Although the permeabilities of the pure PVA membranes in the two different media were not significantly different, the diffusivity, $D$, of CT in the membrane was lower when Hanford water was used, with a proportionally higher membrane-water partition coefficient, $H$, as shown in Table 2. Though the cause of this result is not known, it is the permeability, $P$, that influences the lag time in a reactive membrane. Therefore, any differences observed in the ability of reactive membranes to increase the lag time are not the result of differences in the physical properties of the membranes themselves, but due instead to differences in the reactivity of the $\mathrm{Fe}^{0}$ nanoparticles when exposed to the different types of aqueous media.

Much of the difference observed between the effective iron concentrations in the Tris buffer and the Hanford water is likely the result of the high chloride ion content and stable $\mathrm{pH}$ in the buffer solution, compared to the Hanford water $\left(6.43 \times 10^{-4} \mathrm{M} \mathrm{Cl}^{-}, \mathrm{pH} 8.18\right)$. Both factors should destabilize oxide coatings on the iron particles and facilitates corrosion/reactivity of the iron in the Tris buffer compared to the Hanford water sample.

Overall, the results on the effect of the groundwater matrix on membrane performance are mixed. Based on the results presented, we would expect the iron containing membrane performance (i.e. lag time) to be reduced by approximately a factor of two compared to the performance using a Tris buffer solution. With Hanford water, the performance of the CST containing membrane, improved by a factor of three. We are unsure about the cause of this difference. 


\section{High-Density Polyethylene Membrane Containing Fe ${ }^{0}$ as a Contaminant Barrier}

The work above demonstrated that polyvinyl alcohol (PVA) membranes containing $\mathrm{Fe}^{0}$ nanoparticles substantially delayed the breakthrough of several oxidized contaminants, including carbon tetrachloride (CT). While PVA is a good model system, it is not a practical material because of its low mechanical strength, high water permeability, poor processability, and biodegradability. In this portion of the research, HDPE and nano-scale $\mathrm{Fe}^{0}$ were used to synthesize a new reactive membrane ( $\left.\mathrm{Fe}^{0} / \mathrm{HDPE}\right)$. The barrier properties of this membrane were tested against $\mathrm{CT}$ in diaphragm cell diffusion experiments.

The results are divided into two sections: one for non-reactive membranes and the other for reactive membranes. In the former, the diffusion coefficient and the partitioning coefficient are calculated from breakthrough curves. This partitioning coefficient is compared with the corresponding value from sorption experiments. In the latter, effects of $\mathrm{Fe}^{0}$ and glycerol on the breakthrough lag time are presented and discussed.

\section{Diffusion and partition coefficients}

Table 4 shows a summary of $D$ and $H$ for HDPE membranes. The breakthrough experiments determined $D=1.34 \pm 0.22 \times 10^{-13} \mathrm{~m}^{2} / \mathrm{s}$ and $H=25.8 \pm 13.8$ for CT.

Table 4. Diffusion and partition coefficients of CT and CF.

\begin{tabular}{ccc}
\hline & $\begin{array}{c}D \\
\left(\mathrm{~m}^{2} / \mathrm{s}\right)\end{array}$ & $\begin{array}{c}H \\
(-)\end{array}$ \\
\hline \multirow{2}{*}{$\mathrm{CT}$} & $1.34 \pm 0.22 \times 10^{-13}$ & ${ }^{\mathrm{b}} 105.4 \pm 7.9$ \\
& & $25.8 \pm 13.8$ \\
$\mathrm{CF}$ & ${ }^{\mathrm{a}} 4.90 \pm 1.98 \times 10^{-13}$ & ${ }^{\mathrm{a}} 18.89 \pm 8.26$ \\
& $3.00 \pm 0.35 \times 10^{-13}$ & $6.0 \pm 0.2$
\end{tabular}

${ }^{a}$ From the sorption experiments of Joo et al. (2004).

${ }^{b}$ From sorption experiments in this study.

These values are based on averages over $D-H$ pairs calculated from each breakthrough curve. The corresponding breakthrough curves are normalized and plotted on Figure 14. These data reveal reproducible determination of $D$ (x-intercept) and large variability in $P$ (slope). Because $H$ is calculated from $P$ and $D$, this will lead to large errors in $H$ using this method. There is no trend in $P$ variation with respect to either $C_{u p}$ or $L$. The sorption experiments produced larger and more consistent values for $H(105.4 \pm 7.9)$. These experiments also gave $H$ values independent of membrane thickness as shown in Figure 15. This suggests that the adsorption of $\mathrm{CT}$ on the membrane surface is small compared with partitioning into the membrane. No decrease of CT concentration was observed in a control bottle to which no membrane was added. These findings indicate that the $H$ value from the sorption experiment is more accurate and precise than that from the breakthrough experiment. Compared with $\mathrm{CT}, \mathrm{CF}$ has larger $D$ and smaller $H$ values. This is consistent with the molecular properties because a smaller molecule (CF) diffuses faster, and the more hydrophobic molecule (CT) partitions more strongly into the organic polymer. The $H$ and $D$ values obtained here are comparable to those for other small, halogenated molecules in HDPE (Rowe et al., 1995; Joo et al., 2004). 


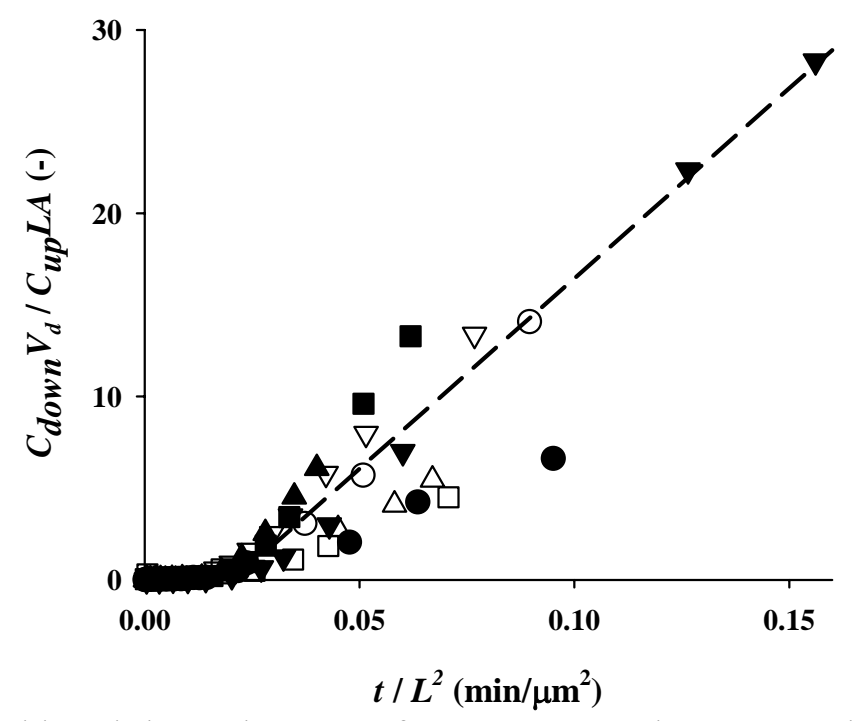

Figure 14. Normalized breakthrough curves for HDPE membranes. Each symbol represents one breakthrough experiment, and eight experiments are shown. The ranges of $C_{u p}$ and $L$ are 746$2222 \mu \mathrm{M}$ and $88-170 \mu \mathrm{m}$, respectively. The dashed line corresponds to $H=25.8$ and $D=$ $1.34 \times 10^{-13} \mathrm{~m}^{2} / \mathrm{s}$.

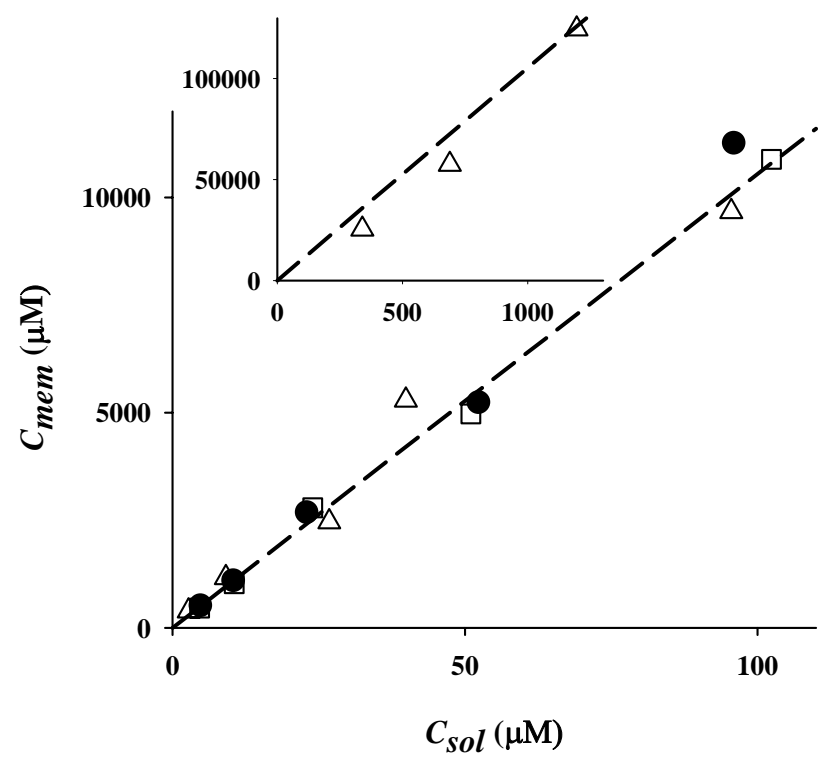

Figure 15. Membrane-water partitioning isotherm: Average thickness of the membrane pieces $=$ $208 \mu \mathrm{m}(\bullet), 106 \mu \mathrm{m}(\square)$, and random thickness about 100-200 $\mu \mathrm{m}(\Delta)$. The inset shows an extended concentration range. The dashed lines indicate $C_{m e m}=105 C_{s o l}$ 
Lag times for reactive membranes

Table 5 summarizes the conditions and results for the breakthrough experiments for membranes containing $\mathrm{Fe}^{0}$. Two experiments without $\mathrm{Fe}^{0}$ (A, B) are also shown for comparison. $\mathrm{Fe}^{0} / \mathrm{HDPE}$ membranes did not always give increased lag times.

Table 5. Summary of experimental conditions and results for breakthrough experiments.

\begin{tabular}{llllllll}
\hline experiment & $\begin{array}{l}\mathbf{L} \\
(\mu \mathrm{m})\end{array}$ & $\begin{array}{l}\mathrm{C}_{\mathrm{up}} \\
(\mu \mathrm{M})\end{array}$ & $\begin{array}{l}\mathrm{V}_{\mathrm{d}} \\
(\mathrm{mL})\end{array}$ & $\begin{array}{l}\mathrm{Fe}^{0}: \text { HDPE } \\
(\mathrm{g} / \mathrm{g})\end{array}$ & $\begin{array}{l}\text { glycerol : HDPE } \\
(\mathrm{g} / \mathrm{g})\end{array}$ & $\begin{array}{l}\text { apparent } \mathbf{P} \\
\left(\mathrm{m}^{2} / \mathrm{s}\right)\end{array}$ & $\begin{array}{l}\mathrm{t}_{\mathrm{ag}} \\
(\mathrm{min})\end{array}$ \\
\hline $\mathrm{A}$ & 106 & 1274 & 12.76 & 0 & 0 & $57.1 \times 10^{-13}$ & 261 \\
$\mathrm{~B}$ & 127 & 1047 & 12.12 & 0 & 0.044 & $98.7 \times 10^{-13}$ & 226 \\
$\mathrm{C}$ & 111 & 1156 & 12.83 & 0.247 & 0 & $47.8 \times 10^{-13}$ & 151 \\
$\mathrm{D}$ & 135 & 1159 & 12.83 & 0.247 & 0 & $47.9 \times 10^{-13}$ & 116 \\
$\mathrm{E}$ & 93 & 931 & 12.83 & 0.053 & 0.039 & $16.2 \times 10^{-13}$ & 293 \\
$\mathrm{~F}$ & 106 & 874 & 12.83 & 0.053 & 0.039 & $22.2 \times 10^{-13}$ & 269 \\
$\mathrm{G}$ & 166 & 737 & 12.83 & 0.247 & 0.217 & $14.6 \times 10^{-13}$ & 9420 \\
$\mathrm{H}$ & 269 & 798 & 12.83 & 0.247 & 0.217 & $13.5 \times 10^{-13}$ & 18900 \\
$\mathrm{I}$ (used H) & 269 & 1151 & 12.83 & - & - & $111.9 \times 10^{-13}$ & 0 \\
\hline amount of glycerol removable from the membrane using the method described in the text. & & &
\end{tabular}

As shown in Figure 16, membranes with $\mathrm{Fe}^{0}$ but without any additive (i.e. glycerol) are not statistically different from a HDPE membrane. This suggests no effect of $\mathrm{Fe}^{0}$ on the CT breakthrough. Only traces of CF were detected at the end of experiments $\mathrm{C}$ and D. This suggests that $\mathrm{Fe}^{0}$ is not reactive in a totally hydrophobic and water-free environment. Another important finding here is no measurable effect of adsorption. Possible adsorption of CT on high surface area nano- $\mathrm{Fe}^{0}$ particles, which might have a similar effect to reaction, is not enough to increase the lag time.

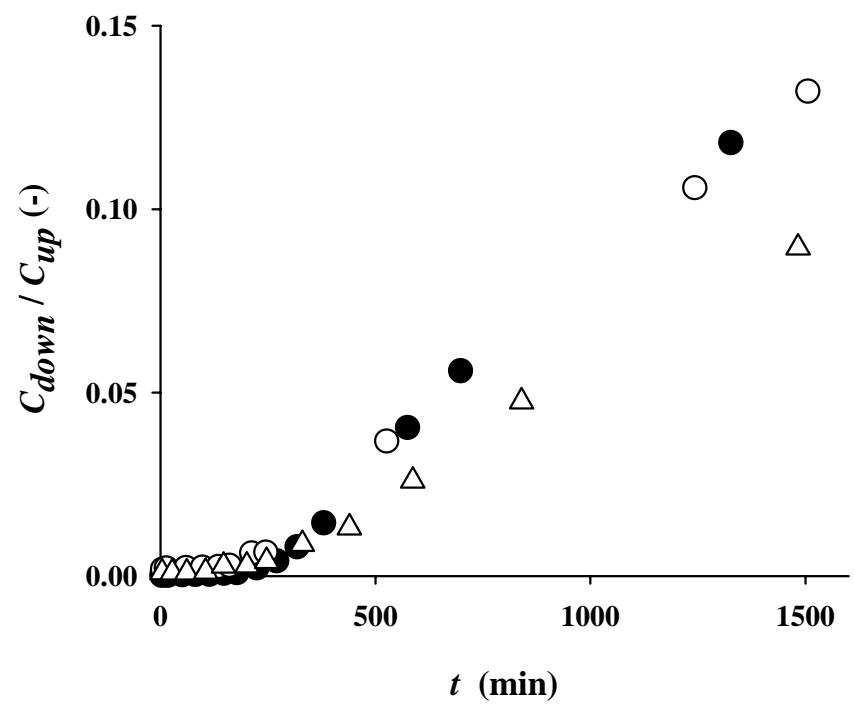

Figure 16. Breakthrough curves for an $\mathrm{HDPE}$ membrane (•; A in Table 2$)$ and $\mathrm{Fe}^{0} / \mathrm{HDPE}$ membranes $(\mathrm{O} ; \mathrm{C}, \Delta ; \mathrm{D})$ prepared without glycerol. 
Experiments using the membranes containing glycerol-coated $\mathrm{Fe}^{0}$ gave different results. Figure 17 shows four breakthrough curves: an HDPE-glycerol membrane (B in Table 5), two $\mathrm{Fe}^{0} / \mathrm{HDPE}$-glycerol membranes $(\mathrm{G}, \mathrm{H})$, and a previously-used $\mathrm{Fe}^{0} / \mathrm{HDPE}$-glycerol membrane (I). The HDPE-glycerol membrane leaks at about the same time as the pure HDPE membrane (A in Figure 16). The $\mathrm{Fe}^{0} / \mathrm{HDPE}$-glycerol membranes successfully increased $t_{\text {lag }}$ by more than 13-16 fold compared to pure HDPE when adjusting for differences in $L$ and $C_{u p}$. The primary reaction product $\mathrm{CF}$ was observed in both the upstream and downstream cells. The results indicate that modification of polymer by a hydrophilic additive such as glycerol stimulates the reaction in the hydrophobic polymer matrix.

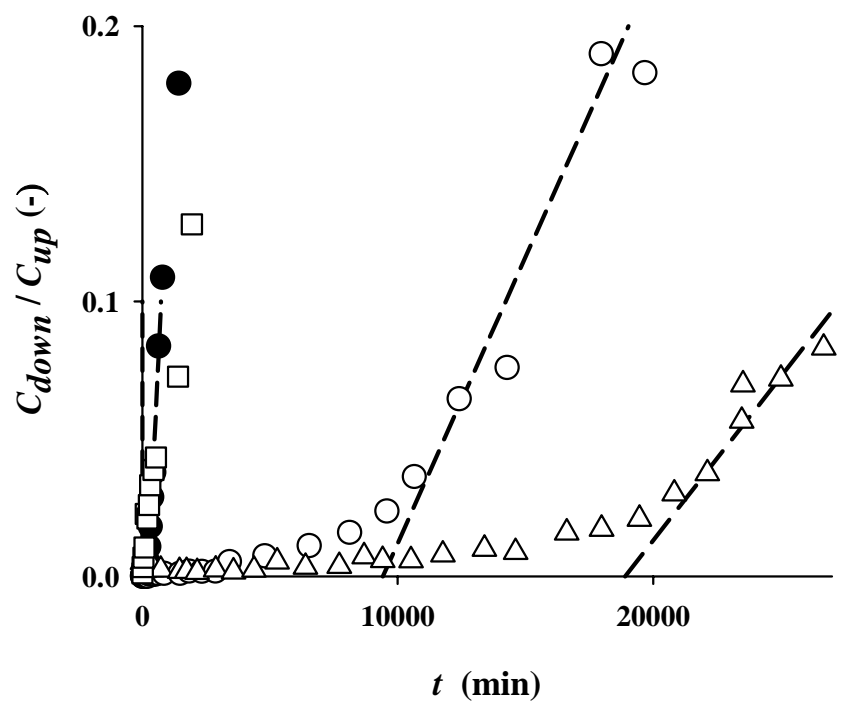

Figure 17. Breakthrough curves for $\operatorname{HDPE}(\bullet ; \mathrm{B}$ in Table 2$), \mathrm{Fe}^{0} / \mathrm{HDPE}(\mathrm{\circ} ; \mathrm{G}, \Delta ; \mathrm{H})$, and an exhausted $\mathrm{Fe}^{0} / \mathrm{HDPE}$ membrane ( $\square$; I) prepared with glycerol. The dashed lines indicate the regression used to determine the lag time.

Experiments with lower $\mathrm{Fe}^{0}$ and glycerol loadings ( $\mathrm{E}$ and $\mathrm{F}$ ), on the other hand, did not show a measurable effect on the lag time even though glycerol was used. This suggests there is a minimum level of glycerol necessary to allow reaction. It is difficult to add a sufficient amount of glycerol to the membrane when the $\mathrm{Fe}^{0}$ loading is small, resulting in insufficient modification of the polymer. For this reason, making an effective reactive membrane with a small $\mathrm{Fe}^{0}$ loading is challenging for this polymer-additive combination.

Measured concentrations of $\mathrm{CT}$ in the upstream cell $\left(C_{u p}\right)$ for experiments $\mathrm{G}$ and $\mathrm{H}$ decreased due to reaction and sorption even with a large upstream cell. The mass balance (sum of the CT and CF masses in the both cells divided by $V_{u p}$ ) also decreased to a lesser extent (10-15 $\%)$. This is due to production of minor products such as methylene chloride and methane, and potentially a small amount of leakage from the system. A time-averaged value of $C_{u p}$ was used for analysis of the data, although $C_{u p}$ should ideally be constant to apply eq 2 .

Figure 18 displays the $\mathrm{CF}$ concentration in the downstream cell for experiment $\mathrm{G}$. The concentration reached a peak of $750 \mu \mathrm{M}$ when the CT breakthrough occurred ( $\sim 9500 \mathrm{~min})$ and then decreased. While the reaction of $\mathrm{CT}$ and $\mathrm{Fe}^{0}$ proceeds in the membrane, there is a concentration peak of $\mathrm{CF}$ within the membrane at the moving reaction front. Thus, there is a driving force for $\mathrm{CF}$ diffusion in both directions, into both cells. Once the $\mathrm{Fe}^{0}$ in the membrane is 


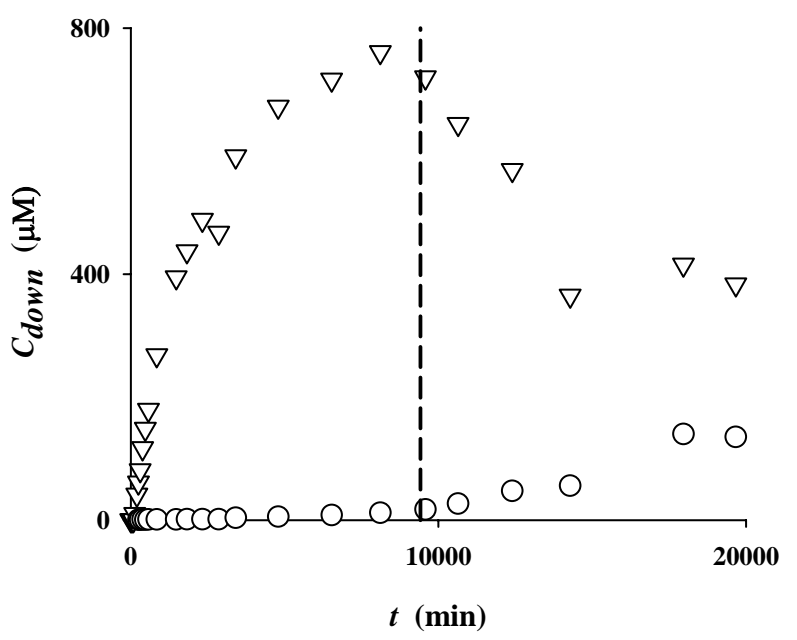

Figure 18. Concentration of $\mathrm{CF}(\Delta ; \mathrm{G}$ in Table 2$)$ in the downstream cell. The corresponding concentration of CT (O) is also shown. The dotted line indicates the breakthrough time.

exhausted, CF starts to migrate from the small-volume downstream cell to the large-volume upstream cell where CF is diluted by the large volume. This is explored further in the final section of the results reported below. Experiments using a mixture of CT and CF as solutes suggested that these two species do not interfere with the diffusion of one another.

Although glycerol was expected to increase the permeability by introducing free volume, the effects of $\mathrm{Fe}^{0}$ and glycerol on the permeability of the membranes are not clear. For example, the apparent steady-state slopes for the reactive membranes after breakthrough (Figure 17) imply that the permeability of these membranes is about $1.4 \times 10^{-12} \mathrm{~m}^{2} / \mathrm{s}$, lower than that of HDPE. This is not true: the smaller slope occurs because the steady-state slope has not yet been reached. An additional experiment with an exhausted $\mathrm{Fe}^{0} / \mathrm{HDPE}$-glycerol membrane (I in Table 2) showed no lag time and a clear breakthrough. The apparent permeability obtained in this experiment is about $1.1 \times 10^{-11} \mathrm{~m}^{2} / \mathrm{s}$, which is high compared to $1.4 \times 10^{-12} \mathrm{~m}^{2} / \mathrm{s}$. This results from $\mathrm{CT}$ accumulation in the membrane during the first run (experiment $\mathrm{H}$ ), which is discharged into the downstream cell in the unsteady state of experiment I. From this interpretation and considering the large variability of $P$ for HDPE membranes, accurate determination of the apparent permeability for the reactive membranes is difficult. For the purpose of lag-time analysis, the average permeability for HDPE, $3.5 \times 10^{-12} \mathrm{~m}^{2} / \mathrm{s}$, was adopted.

Cross-sections of an $\mathrm{Fe}^{0} / \mathrm{HDPE}$ membrane ( $\mathrm{G}$ in Table 5) were analyzed using a reflective microscope before and after the breakthrough experiment. The images show an obvious uniform color change from black to yellow/orange, proving that at least the surface of $\mathrm{Fe}^{0}$ in the membrane was oxidized upon reaction with CT. Dissolution of iron into the diaphragm cells was measured, and it was small compared to the amount of CT degraded. This suggests that a fraction of the iron near the surface of the membrane is dissolved into the solutions, while the remaining oxidized iron is trapped and/or precipitated inside the membrane. Moreover, much of the iron appears to not react, because the experimental lag times are almost 40 times smaller than the lag times predicted based on the iron loading. The ratio of the experimental to estimated lag times suggests that only $2.5-3.0 \%$ of the iron was used. This implies passivation of iron in the HDPE/glycerol matrix and the possibility of iron deactivation (e.g., oxidation) during the membrane synthesis process. 


\section{The Third Parameter in Reactive Barrier Films}

The barrier quality of a conventional barrier membrane is typically measured with one parameter-its permeability. We have shown that the addition of reactive groups can raise the membrane's lag time to equal or greater status - assuming that the reaction is fast relative to diffusion. Two membranes with similar lag times, however, can allow different solute fluxes, depending on their reaction rate. We need, then, a third parameter to quantify this amount of solute. In this section, we introduce two candidates for this parameter, the Leakage and the Kill Time. Here we use computer models to calculate breakthrough plots for different membranes, quantify their curvature with our new parameters, and correlate them to the physical parameters of these model membranes. Symbols in this section are slightly modified from those in other sections of the report. Here, $L$ is leakage (rather than membrane thickness), the thickness of the membrane is $\ell, C_{X o}$ is the upstream concentration, $C_{X D}$ is the downstream concentration, $C_{Y O}$ is the initial concentration of immobilized reagent, $V_{2}$ is the downstream volume, and $t_{L}$ is the lag time.

\section{Curvature Quantification}

We can classify barrier membranes into two categories. In one category are packages that react quickly with the target solute. In the second category are films that react slowly with the target solute. The reaction rate in these latter systems is typically a function of the solute concentration in the barrier film.

In the first case, we are interested simply in the total amount of solute $Q$ which has entered the package. Since $Q$ grows without limit, we must define a time at which $Q$ is measured. More practically, we must define a maximum allowable $Q$ and measure the amount of time required to reach this $Q_{\operatorname{Max}}$. For instance, a given amount of pollutant that is deemed unacceptable. This amount of pollutant is our $Q_{\operatorname{Max}}$. The lifetime of our barrier is the time required to reach this $Q_{\operatorname{Max}}$. We call this time the "kill time", $t_{K}$.

In the second case, we are interested not only in the amount of solute in the package, but also how long it is there (i.e., concentration times exposure time). For example, 100ppm of pollutant may do as much damage in ten days as $1000 \mathrm{ppm}$ of pollutant will do in one day. We are interested, then, not in $Q$, but rather in $\int Q d t$. Of course, $\int Q d t$ also grows without limit. However, we know that a given membrane will approach a predictable breakthrough asymptote regardless of its reaction rate. At large times, then, $Q$ of any membrane is the same as that of an otherwise identical film with an infinite reaction rate, $Q_{\text {Ideal }}$.

$$
\underset{t \rightarrow \infty}{\boldsymbol{Q}}=\boldsymbol{Q}_{\text {Ideal }}=\frac{A D H C_{X o}}{\ell} \mathrm{Hv}\left(\boldsymbol{t}-\boldsymbol{t}_{\mathbf{L}}\right)
$$

where $\mathrm{Hv}$ is the Heaviside function. That being true,

$$
\frac{d}{d t}\left(\int Q d t\right)=\frac{d}{d t}\left(\int Q_{\text {Ideal }} d t\right)
$$

Thus at large times, $\int Q d t$ will grow at the same rate as $\int Q_{\text {Ideal }} d t$, a rate which we can calculate. The difference between $\int Q d t$ and $\int Q_{\text {Ideal }} d t$, then, is finite. We call this difference the 'leakage', $L$. In physical terms, this is a measure of how much more damage will occur if our real membrane is used rather than an ideal one. 
Each parameter has advantages over the other, and we could philosophize over their relative usefulness for far too long. In general, is it more useful to have a data point at a single chosen instant, or an average of data points out to infinity (or at least out to the steady-state flux regime)? The answer depends on the application. For our purposes, the leakage is the easier parameter to use, since it requires one less parameter to correlate (the kill time depends also on $Q_{\text {Max }}$ ) and is less likely to be skewed by error (being an average over many points in a curve, rather than just one).

\section{Non-dimensionalization of Kill Time, Leakage}

At first glance, calculation of the kill time and leakage should be straightforward. To find $t_{K}$, we set a $Q_{M a x}$ and scan the breakthrough plot for the time at which $Q_{\operatorname{Max}}$ is achieved. This is $t_{K}$. For the leakage, we regress the plot at large time to get our asymptote function. We integrate this function over time to the large time of the regression analytically. We then numerically integrate $Q$ out to the same large time and subtract off the analytic integration. The result is $L$.

There are complications, however. First of all, none of our breakthrough plots are of $Q$ vs. $t$. Our experimental plots are of $C_{X D}$ vs $t$, and knowing $V_{2}$ we can calculate $Q$ vs. $t$. Our computer-generated plots use the non-dimensional variables $\Psi_{\mathrm{XD}}\left(=C_{X C} / H C_{X o}\right)$ and $\tau\left(=D t / \ell^{2}\right)$; they must first be re-dimensionalized (by $H C_{X o}$ and $\ell^{2} / D$, respectively). However, in our computer plots, we never specified $H C_{X o}$, or $\ell^{2} / D$, or even $V_{2}$. We have no way of getting a plot of $Q$ vs. t.

Nor should we want to. The purpose of non-dimensionalizing our equations is to minimize the number of parameters we correlate to $t_{K}$ and $L$. But if those parameters are all dimensionless, then the kill time and leakage must be dimensionless as well. We can nondimensionalize the kill time in the same manner as our other times - multiplying it by $D / \ell^{2}$.

$$
\tau_{\boldsymbol{K}}=\frac{\boldsymbol{D} \boldsymbol{t}_{\boldsymbol{K}}}{\ell^{2}}
$$

To determine $t_{K}$, we also need a dimensionless $Q_{\operatorname{Max}}$, which we'll dub $\Theta_{\text {Max. }}$. This is more arbitrary; we have no precedent for dealing with moles. When dealing with units of solute concentration, we divide by $H C_{X o}$, and can do the same here. That leaves us with units of volume, though. Dividing by the downstream chamber volume seems obvious, since that is the volume with which $Q_{\operatorname{Max}}$ is concerned. We will discover, however, that dividing by the membrane volume $A \ell$ will make the derivation/calculation easier in the long run. Thus,

$$
\Theta_{\text {Max }}=\frac{Q_{\text {Max }}}{H C_{X o} A \ell}
$$

Non-dimensionalization of the leakage, $L$, is similarly tricky. It has units of moles time. We can again multiply by $D / \ell^{2}$ to remove the time unit and, as with $Q_{M a x}$, divide by $H C_{X o}$ and $A \ell$ to remove the moles unit, giving us non-dimensional leakage, $\Lambda$.

$$
\Lambda=\frac{L}{(\boldsymbol{A} \ell)\left(\ell^{2} / \mathbf{D}\right)\left(\boldsymbol{H} \boldsymbol{C}_{\mathbf{X o}}\right)}=\frac{\boldsymbol{D} \boldsymbol{L}}{\boldsymbol{A} \ell^{3} \boldsymbol{H} \boldsymbol{C}_{\mathbf{X o}}}
$$

\section{Calculation of Kill Time}

We use normalized versions of simulated breakthrough plots where we plot $\left(V_{2} C_{X D} /\right.$ $\left.A \ell H C_{X O A}\right)$ vs. $\left(\tau-\tau_{L}\right)$. Now, we must divide our $Q_{\operatorname{Max}}$ by $A \ell H C_{X O A}$ (i.e. find $\Theta_{\text {Max }}$ ) and look for 
the corresponding $\left(\tau-\tau_{L}\right)$ value, which is then $\left(\tau_{K}-\tau_{L}\right)$. Knowing $\tau_{L}$, we know $\tau_{K}$, and if we know $\ell^{2} / D$, we can re-dimensionalize to $t_{K}$, if we choose. We have to choose our $\Theta_{\text {Max }}$ directly, rather than calculating it from a $Q_{\operatorname{Max}}$. Once we have $\tau_{K}$, we cannot re-dimensionalize it back to $t_{K}$. We varied $\Theta_{\mathrm{Max}}$ over 23 orders of magnitude, from $10^{-20}$ to $10^{3.5}$. We then scanned each computer-generated breakthrough plot, recording the time at which each $\Theta_{\mathrm{Max}}$ was surpassed. We did not attempt to interpolate between data points, which are 0.01 time units apart; the first time point with a $\Theta$ larger than $\Theta_{\mathrm{Max}}$ was recorded as the corresponding $\tau_{K}$.

\section{Calculation of Leakage}

To find the leakage of a breakthrough plot, we integrate both the plot, $Q$, and the plot of a similar, but ideal membrane, $Q_{\text {Ideal }}$, and take their difference

$$
L=\int_{0}^{t} Q d t-\int_{0}^{t} Q_{\text {Ideal }} d t
$$

We recall that $Q_{I d e a l}$ is simply the asymptote which $Q$ approaches, and that this asymptote can be analytically described by equation 16 . We can also break down $Q$ to $V_{2} C_{X D}$.

$$
L=\int_{0}^{t} V_{2} C_{X D} d t-\int_{t_{L}}^{t} \frac{A D H C_{X o}}{\ell}\left(t-t_{L}\right) d t
$$

We divide by $\left(A D H C_{X O} / \ell\right)$ giving

$$
\frac{\ell L}{A D H C_{X o}}=\int_{0}^{t} \frac{\ell V_{2} C_{X D}}{A D H C_{X o}} d t-\int_{t_{L}}^{t}\left(t-t_{L}\right) d t
$$

Now all we need to know to find $L$ is the slope of the asymptote and the function $\left(\ell V_{2} C_{X D}\right)$ $\left.A D H C_{X o}\right)$ vs. $\left(t-t_{L}\right)$.

$$
\frac{L}{A \ell H C_{X o}}=\int_{0}^{t} \frac{V_{2} C_{X D}}{A \ell H C_{X o}} d t-\int_{t_{L}}^{t}\left(\tau-\tau_{L}\right) d t
$$

Since our independent variable is $\left(\tau-\tau_{L}\right)$, it would be easiest to integrate over $\tau$ than $\mathrm{t}$ :

$$
d \tau=d\left(\frac{D t}{\ell^{2}}\right)=\frac{D}{\ell^{2}} d t
$$

which leaves us with

$$
\frac{\boldsymbol{D L}}{\boldsymbol{A} \ell^{3} \boldsymbol{H} \boldsymbol{C}_{X o}}=\int_{0}^{\tau} \frac{\boldsymbol{V}_{2} \boldsymbol{C}_{X D}}{\mathbf{A} \ell \boldsymbol{H} \boldsymbol{C}_{X o}} \boldsymbol{d} \tau-\int_{\tau_{L}}^{\tau}\left(\tau-\tau_{L}\right) \boldsymbol{d} \tau
$$

Recalling the definition of our dimensionless leakage $\Lambda$,

$$
\Lambda=\int_{0}^{\tau} \frac{\boldsymbol{V}_{2} \boldsymbol{C}_{X \boldsymbol{D}}}{\boldsymbol{A} \ell \boldsymbol{H} \boldsymbol{C}_{X o}} \boldsymbol{d} \tau-\int_{\tau_{\mathrm{L}}}^{\tau}\left(\tau-\tau_{L}\right) \boldsymbol{d} \tau
$$

Converting equation 27 to a numeric summation over discrete data,

$$
\Lambda=\sum_{j} \frac{\boldsymbol{V}_{2} \boldsymbol{C}_{X D, j}}{\boldsymbol{A} \ell \boldsymbol{H} \boldsymbol{C}_{X o}} \Delta \tau-\frac{1}{2}\left(\tau-\tau_{L}\right)^{2}=\Delta \tau \sum_{j} \Gamma \Psi_{X D, j}-\frac{1}{2}\left(\tau-\tau_{L}\right)^{2}
$$


Modeling Results: Leakage

We ran both particulate and non-particulate models with a variety of parameter values, varying both $\Phi\left(=\frac{k C_{Y_{o}} \ell^{2}}{D}\right.$, where $k$ is the reaction rate constant $)$ and $\gamma\left(=\frac{C_{Y_{o}}}{H C_{X o}}\right)$ by orders of magnitude (e.g., we would set each value equal to $10^{1}, 10^{1.5}, 10^{2}$, etc.). $\Gamma\left(=\frac{V_{2}}{A \ell}\right)$ was set to 1.0 for all runs, since the effect of $\Gamma$ can be determined analytically. $\Gamma$ is essentially just a constant by which the ordinate of our normalized plots is multiplied. It can be factored directly out of our $\Theta_{\text {Max }}\left(\Theta_{\text {Max }}=\Gamma \Psi_{\mathrm{XD}, \mathrm{Max}}\right)$ and should have a linear effect on $\Lambda$.

Leakage should only depend on two parameters, then, giving it a much simpler analysis than for the kill time, which also depends on $Q_{\operatorname{Max}}$. We present this analysis first. Figure 19 plots $\log (\Lambda)$ vs. $\log (\gamma)$ at various values of $\log (\Phi)$. The figure is striking not only because the plots are so linear, but because they all appear equally spaced with the same slope. Regressions of each plot give slopes ranging from 1.98 to 2.01, with an average of 1.99 . We conclude, then, that

$$
\begin{aligned}
& \log (\Lambda)=(1.99) \log (\gamma)+\text { Const }_{1} \\
& \Lambda=\left(10^{\text {Const }_{1}}\right) \gamma^{1.99}
\end{aligned}
$$

Const $_{1}$ is the intercept of each regression, of course, which progressed from 0.147 at $\log (\gamma)=0.0$ to -4.26 when $\log (\Phi)=4.5$. Regressing Const $_{1}$ vs. $\log (\Phi)$, we find that

$$
\text { Const }_{1}=(-1.10) \log (\Phi)+0.804
$$

with an $\mathrm{R}^{2}$ value of 0.9985 . Plugging this back into Equation 30,

$$
\Lambda=6.37 \gamma^{1.99} \Phi^{-1.10} \cong 6.4 \gamma^{2} \Phi^{-1}
$$

To check $\Lambda$ 's dependence on $\Phi$, Figure 20 plots $\log (\Lambda)$ vs $\log (\Phi)$ at various values of $\log (\gamma)$. As expected, the plots are linear and equally spaced. Regressing them, we find slopes ranging from -0.98 to -1.16 , with an average of -1.10 , consistent with our estimate above.

$$
\begin{aligned}
& \log (\Lambda)=(-1.10) \log (\Phi)+\text { Const }_{2} \\
& \Lambda=\left(10^{\text {Const }_{2}}\right) \Phi^{-1.1}
\end{aligned}
$$

Const $_{2}$, the intercept of each regression between $\log (\Lambda)$ and $\log (\Phi)$, varies linearly with $\log (\gamma)$, and regressing it with $\log (\gamma)$ we find:

$$
\text { Const }_{2}=(2.09) \log (\gamma)+0.665
$$

with an $\mathrm{R}^{2}$ of 0.9999 . Substituting equation 35 into 34 ,

$$
\Lambda=4.62 \gamma^{2.09} \Phi^{-1.1}
$$

While not identical, equations 32 and 36 are quite close, as they should be coming from the same data sets. The meaning and exact value of the coefficient, presumably between 4.6 and 6.4, are not well understood, but the dependence of $\Lambda$ on $\Phi$ and $\gamma$ is clear. Comparing the two equations, we conclude

$$
\Lambda \cong 5 \gamma^{2} \Phi^{-1}
$$




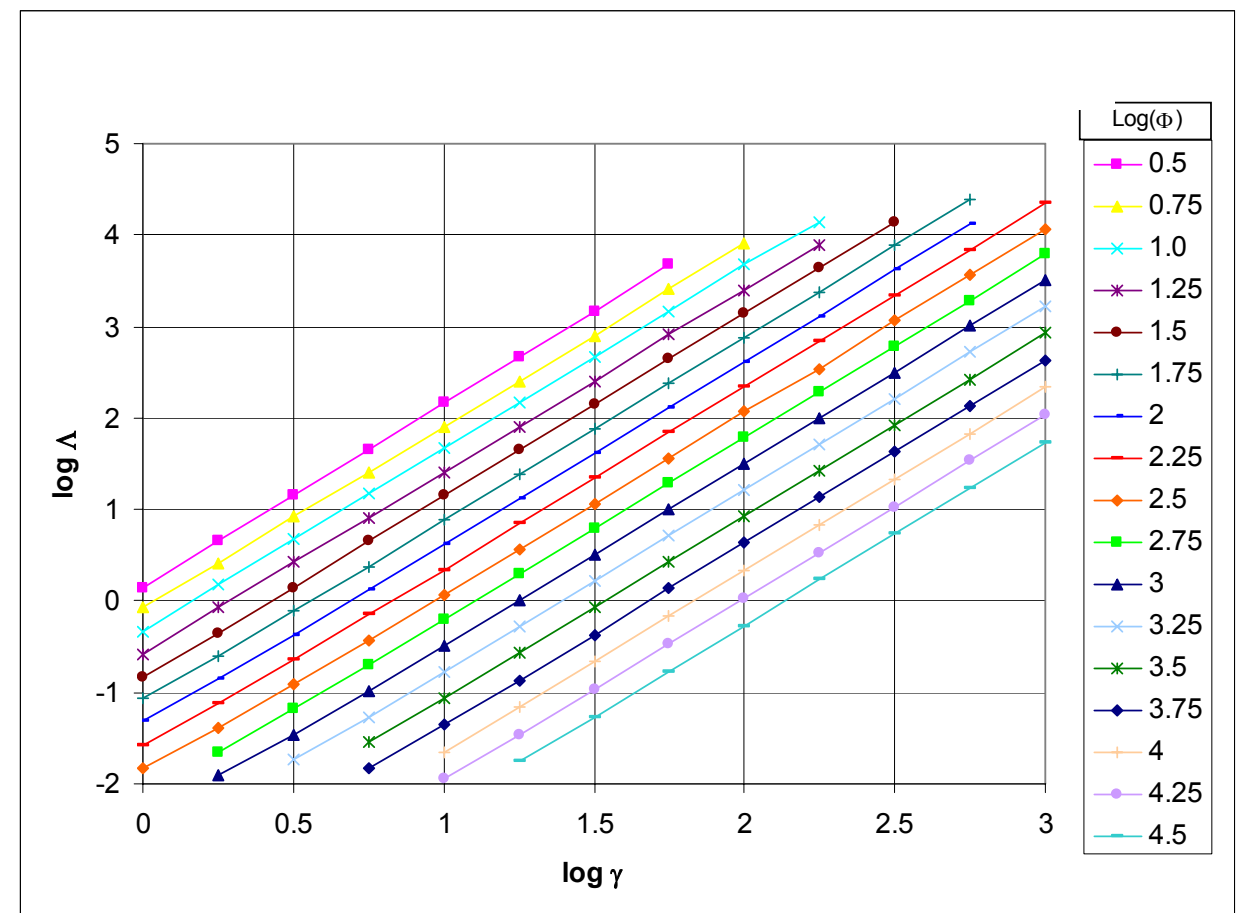

Figure 19. Leakage versus membrane loading.

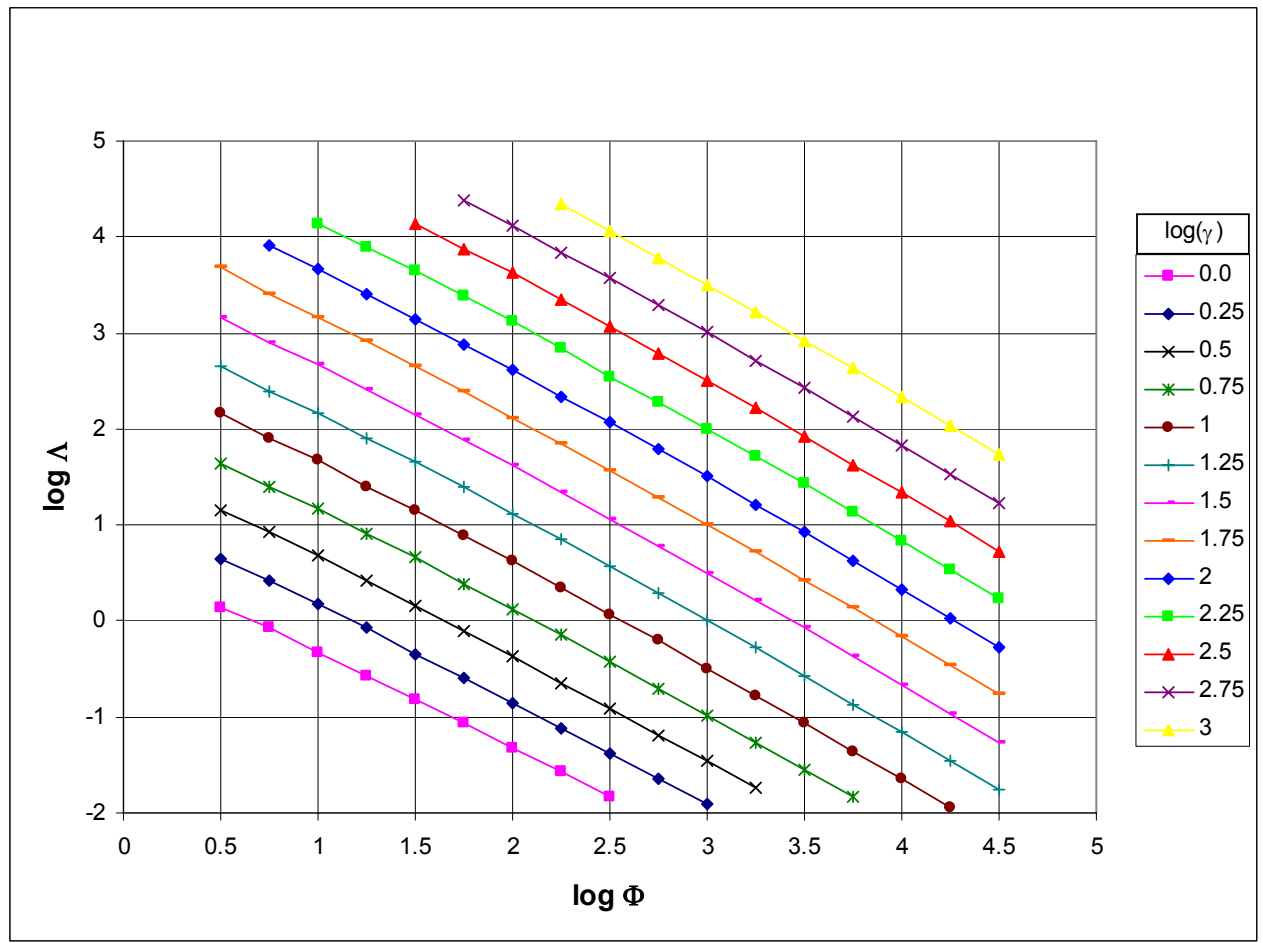

Figure 20. Leakage versus reaction rate (non-particulate). 


\section{Modeling Results: Kill Time}

Analysis of the kill time, $t_{K}$, is more complex. In addition to $\Phi$ and $\gamma$, the kill time also depends on the chosen kill concentration, $\Theta_{\mathrm{Max}}$. Figure 21 plots $\tau_{\mathrm{K}} \mathrm{vs} . \log (\Phi)$ at a variety of $\gamma$ values. We see S-shaped curves with horizontal plateaus on each end.

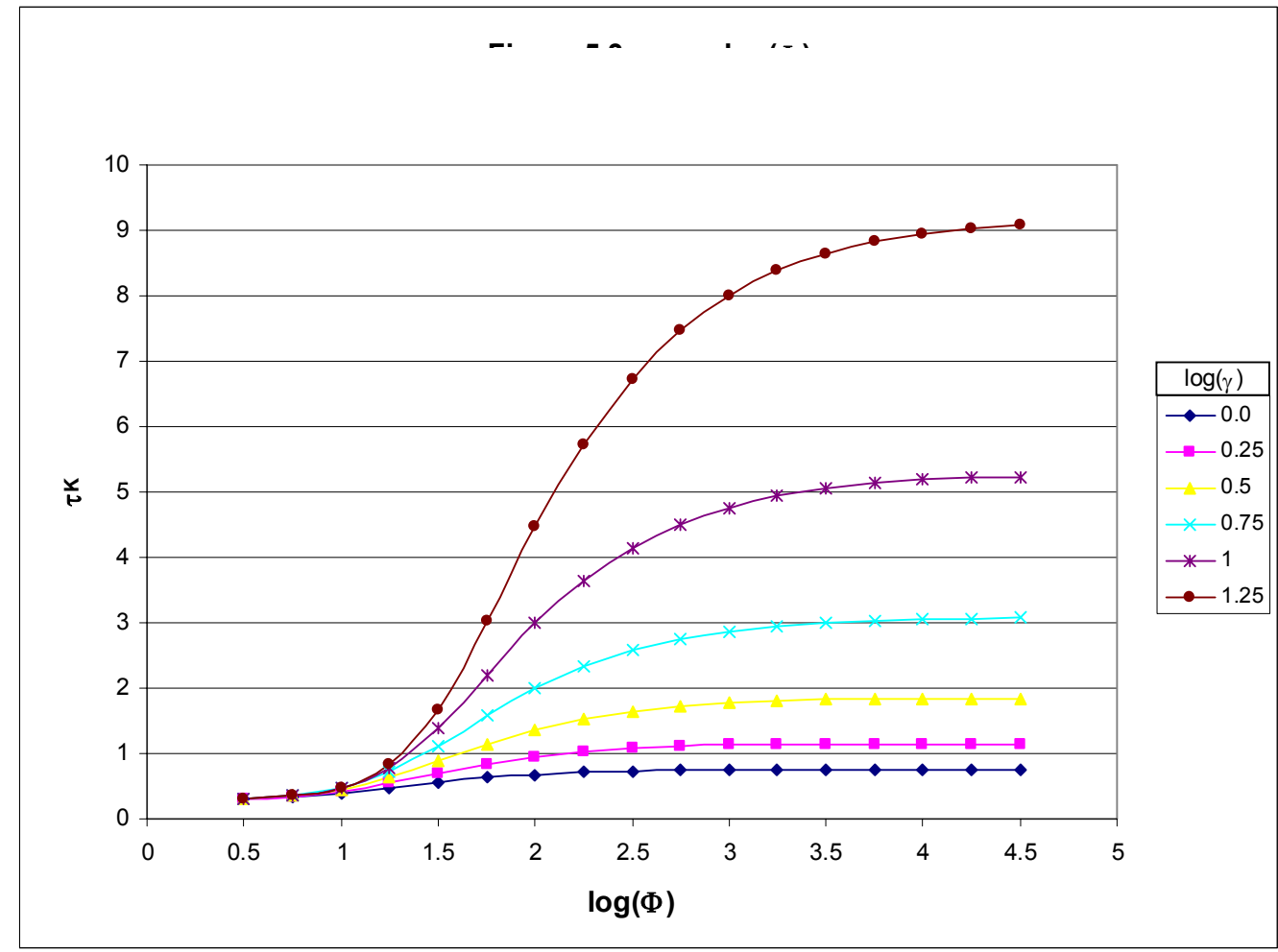

Figure 21. $\tau_{\mathrm{K}}$ versus $\log (\Phi)$ with $\log \left(\Theta_{\mathrm{Max}}=-1\right)$

We should expect this to be the case, since our system has known limits. As $\Phi$ approaches zero, the membrane should behave as an unreactive film. For an unreactive membrane barrier,

$$
\tau_{K}=\Theta_{\text {Max }}+\frac{1}{6}+\frac{2}{\pi^{2}} \sum_{n=1}^{\infty} \frac{(-1)^{n}}{\boldsymbol{n}^{2}} \exp \left(-\boldsymbol{n}^{2} \pi^{2} \tau_{K}\right)
$$

As $\Phi$ approaches infinity, the membrane should behave as an ideal reactive membrane, not allowing any solute to pass until after the lag time, then transmitting solute at steady state.

$$
\tau_{K}=\Theta_{\text {Max }}+\frac{1}{6}+\frac{\gamma}{2}
$$

What intrigues us is the transition between the two limits. There, a small change in $\Phi$ can correspond to a huge change in $\tau_{K}$. Predicting the location and shape of this "Window of Opportunity" is key to intelligent reactive barrier design.

In Figure 21, this window seems to be in the same location regardless of $\gamma$. Making the same plots at various $\Theta_{\text {Max }}$ instead of various $\gamma$, Figure 22 shows that this window location does depend on $\Theta_{\text {Max. }}$. It also shows more clearly that these curves have no inversion symmetry. The departure from the unreactive limit is relatively sharp compared with the slow, gradual approach to the ideal membrane limit. Among the myriad mathematical functions that could give such an 


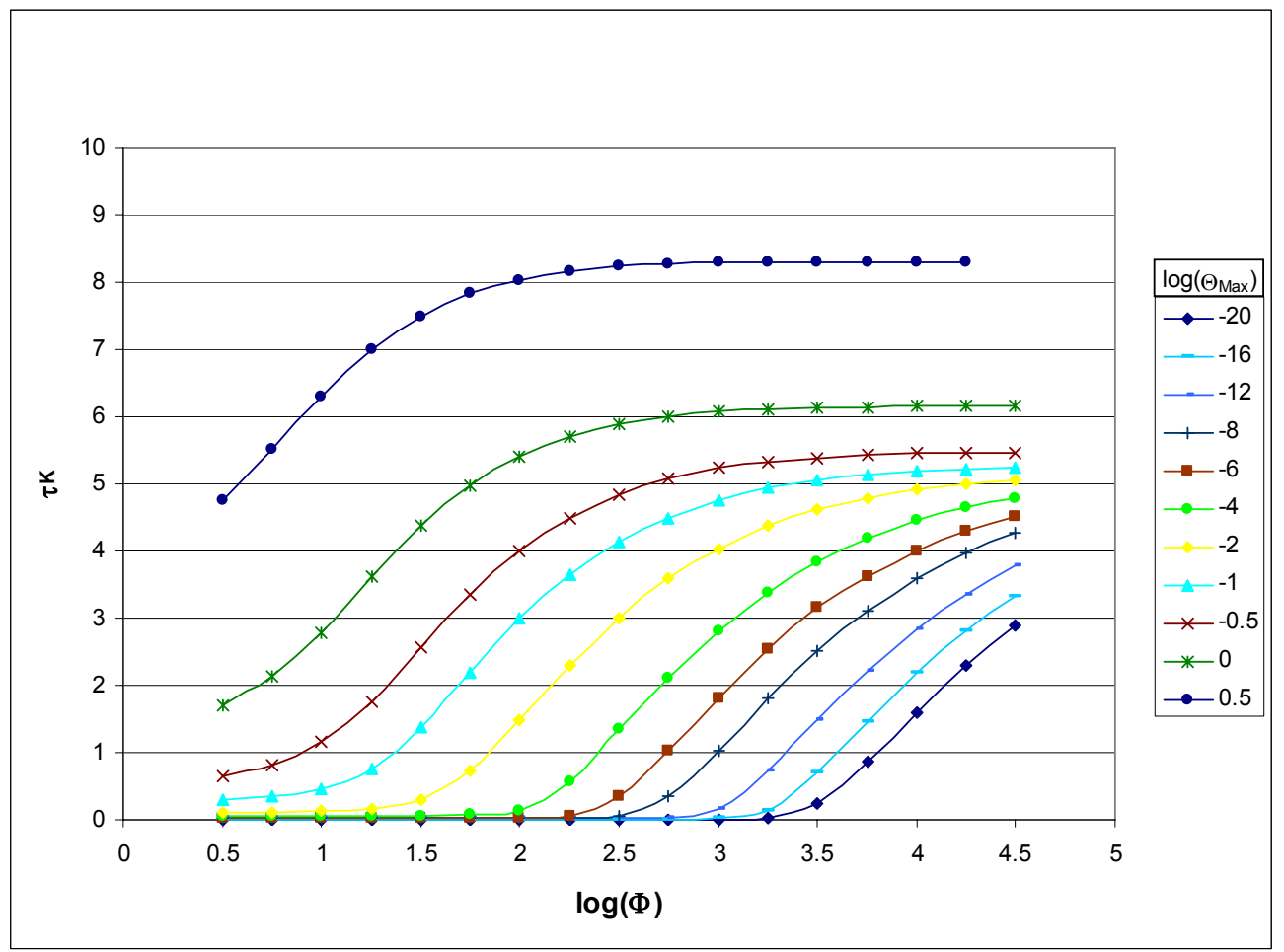

Figure 22. $\tau_{\mathrm{K}}$ versus $\log (\Phi)$ with $\log (\gamma=1)$

asymmetric $\mathrm{S}$-curve is the function $\mathrm{y}(\mathrm{x})=\operatorname{erfc}\left(10^{\mathrm{Ax}}\right)$, where $\mathrm{A}$ is a negative constant and erfc is the complimentary error function:

$$
\boldsymbol{e r f c}(\boldsymbol{x})=\frac{2}{\sqrt{\pi}} \int_{x}^{\infty} \exp \left(-\boldsymbol{u}^{2}\right) \mathbf{d u}
$$

Attempting to fit our curves to this function, we first write:

$$
\tau_{K}=\text { Const }_{1}+\left(\text { Const }_{2}\right) \text { erfc }\left(\text { Const }_{3} \Phi^{\text {Const }_{4}}+\text { Const }_{5}\right)
$$

These are a lot of constants. In truth, they presumably vary with $\gamma$ and $\Theta_{\text {Max }}$, but since we are currently treating $\Phi$ as our only independent variable, we call them constants. The first two determine the $\tau_{\mathrm{K}}$ values for the two plateaus, which we discussed above. Const $t_{1}$, then, is simply the right hand side of equation 38, and Const $_{2}$ is the difference between this and the right hand side of equation 39 .

$$
\begin{aligned}
& \text { Const }_{1}=\Theta_{\text {Max }}+\frac{1}{6}+\frac{2}{\pi^{2}} \sum_{n=1}^{\infty} \frac{(-1)^{n}}{n^{2}} \exp \left(-n^{2} \pi^{2} \text { Const }_{1}\right) \\
& \text { Const }_{2}=\frac{\gamma}{2}-\frac{2}{\pi^{2}} \sum_{n=1}^{\infty} \frac{(-1)^{n}}{n^{2}} \exp \left(-n^{2} \pi^{2} \text { Const }_{1}\right)
\end{aligned}
$$

An unreactive film $(\Phi=0)$ should fall exactly on the unreactive limit, meaning that Const ${ }_{5}$ must be zero. That leaves Const $_{3}$ and Const $_{4}$ for us to determine from our modeling data. We know that Const $_{3}$ must be positive to evaluate the function and Const $_{4}$ must be negative to make the curve transition in the correct direction. 

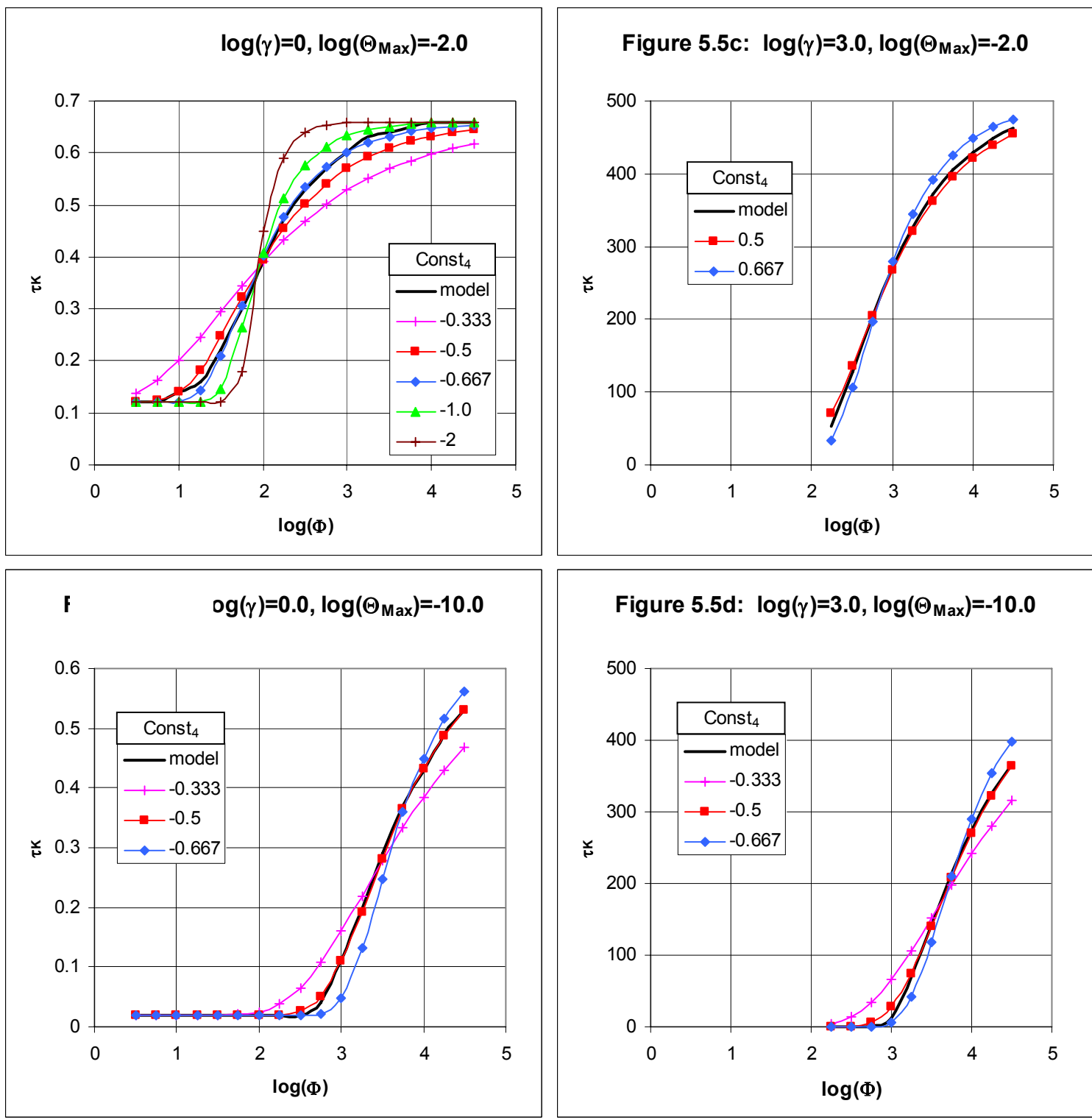

Figure 5.5d: $\log (\gamma)=3.0, \log \left(\Theta_{M a x}\right)=-10.0$

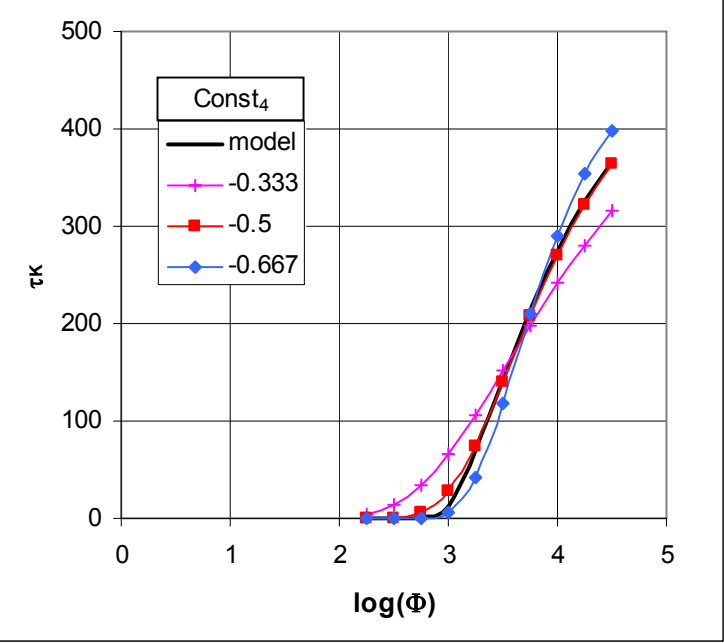

Figure 23: Curve fits to determine Const $_{4}$

Figure 23 plots $\tau_{K}$ vs $\log (\Phi)$ for several sets of model data. For each set, we also plot equation 40, varying Const $_{4}$ from $-1 / 3$ to -2 and using the Const $_{3}$ value which gives the best fit for each. Const ${ }_{4}$ determines the overall sharpness of the transition from one plateau to the other, and we see from Figures 23 that for the best fit, Const ${ }_{4}$ is always between $-1 / 2$ and $-2 / 3$. We may also notice that at small values of $\Theta_{\text {Max }}$ and large values of $\gamma$, Const $_{4}$ is most appropriately set to $-1 / 2$. Since this is the region of parameter space in which we would likely be most interested in the kill time, and since the variation in Const $_{4}$ is so small anyway, we set Const $_{4}$ equal to $-1 / 2$. Our equation, then, is

$$
\tau_{K}=\text { Const }_{1}+\left(\text { Const }_{2}\right) \operatorname{erfc}\left(\text { Const }_{3} \Phi^{-0.5}\right)
$$

where Const $_{1}$ and Const 2 are known (but not written out for brevity), leaving Const ${ }_{3}$ still to be determined. 


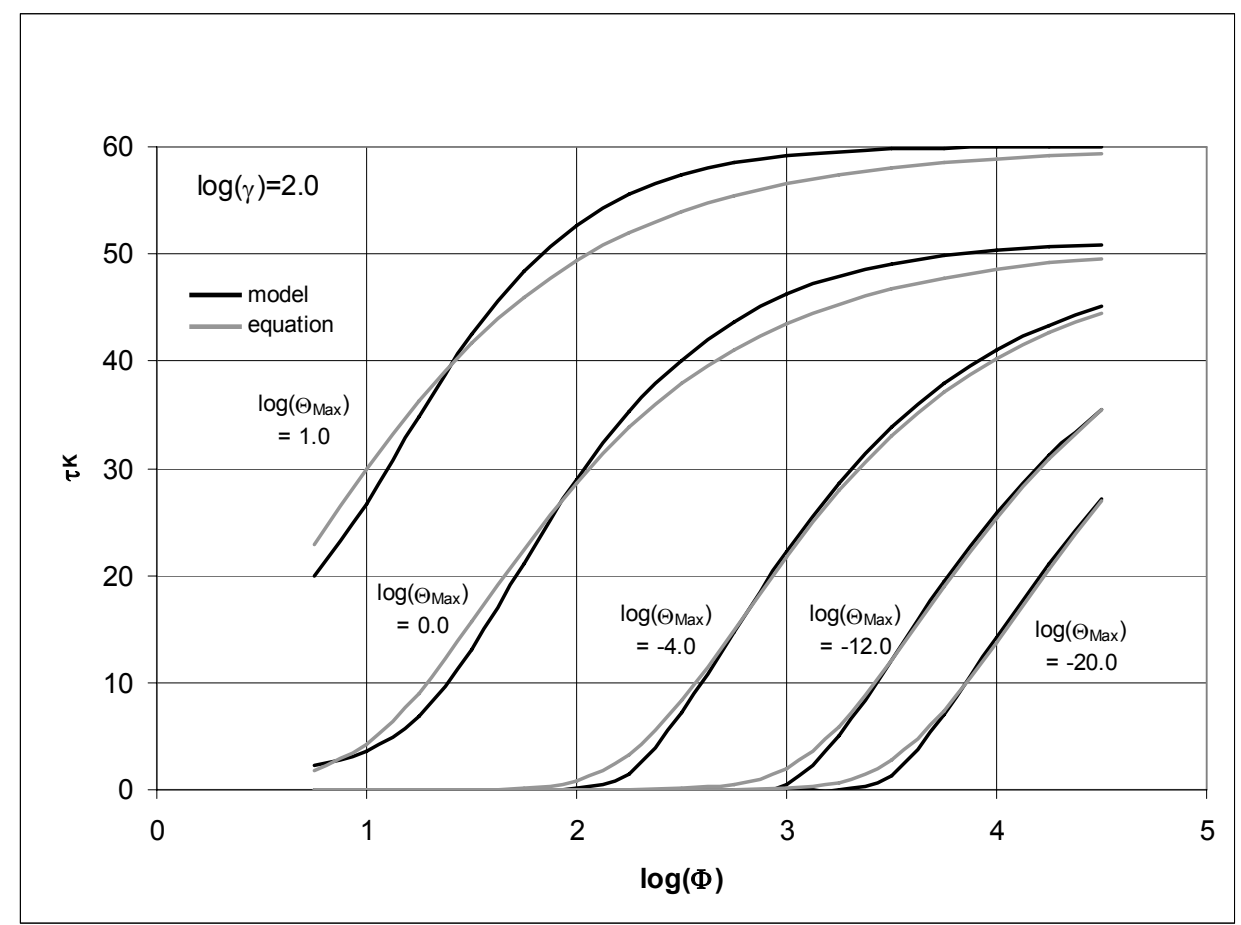

Figure 5.6. Fitting Equation 44 to model data.

Figure 24 plots $\tau_{\mathrm{K}}$ vs $\log (\Phi)$ for a model membrane $(\log (\gamma)=2.0)$ at different $\Theta_{\text {Max. }}$ A different Const $_{3}$ value is used to fit equation 44 to each curve with visible success. Finding the best Const $_{3}$ value for each curve (as determined by a least-squares analysis) and plotting them against $\log \left(\Theta_{\text {Max }}\right)$, Figure 25 shows us a linear relationship between Const $_{3}$ and $\log \left(\Theta_{\text {Max }}\right)$. This relationship breaks down as Const $_{3}$ approaches zero, since equation 44 cannot be evaluated with a negative Const $_{3}$. This corresponds to the regime of large $\Theta_{\text {Max }}$ values, where we know our curve-fit is not as accurate anyway. 


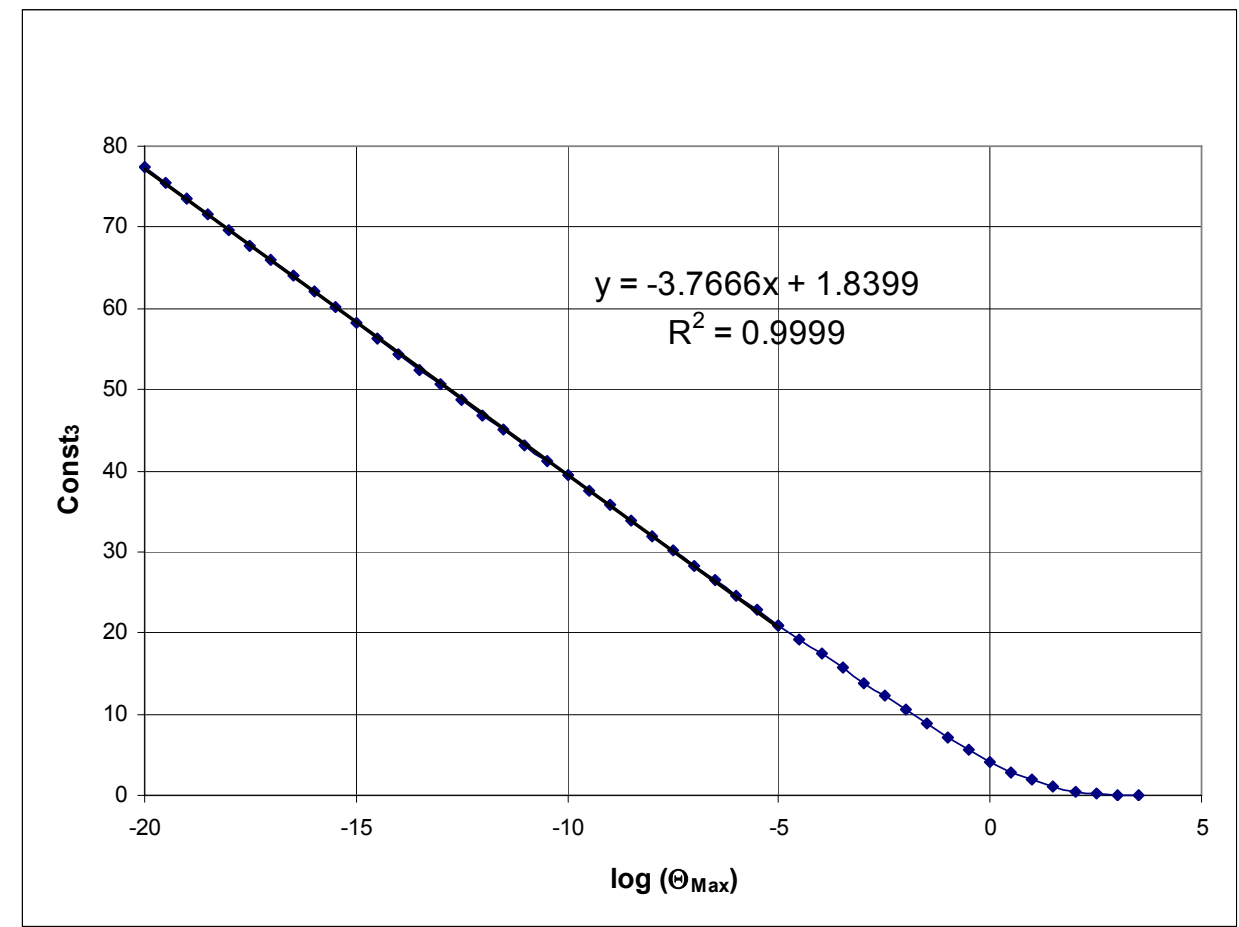

Figure 25. Corrlation of $\mathrm{Const}_{3}$ to $\Theta_{\mathrm{Max}}$

The trendline in Figure 25 regresses only the $\log \left(\Theta_{\text {Max }}\right)$ points from -5 to -20 . Repeating this analysis for several model membrane sets at values of $\log (\gamma)$ from 0.5 to 3.0, we see nearly identical relationships between Const $_{3}$ and $\log \left(\Theta_{\text {Max }}\right)$ each time. Table 6 shows the slope, intercept, and $\mathrm{R}^{2}$ values of each regression. We see that except for very small $\gamma$ values, where little reaction occurs anyway, the relationship between Const $_{3}$ and $\log \left(\Theta_{\mathrm{Max}}\right)$ has a constant slope of -3.76 and an intercept which appears to grow steadily with $\gamma$. In fact, plotting this intercept vs. $\log (\gamma)$, Figure 26 shows us that this intercept varies linearly with $\log (\gamma)$, with a slope of 3.74.

Table 6. Correlations of $\mathrm{Const}_{3}$ and $\log \left(\Theta_{\mathrm{Max}}\right)$

$\begin{array}{cccc}\text { Const }_{3} & =(\text { slope }) \log \left(\Theta_{\text {Max }}\right)+(\text { intercept }) \\ \log (\gamma) & \text { slope } & \text { intercept } & \mathrm{R}^{2} \\ 0.50 & -4.46 & -5.29 & 0.9996 \\ 1.00 & -3.96 & -2.11 & 0.9998 \\ 1.50 & -3.81 & -0.03 & 0.9999 \\ 2.00 & -3.77 & 1.84 & 0.9999 \\ 2.25 & -3.76 & 2.75 & 0.9999 \\ 2.50 & -3.76 & 3.65 & 0.9999 \\ 2.75 & -3.76 & 4.53 & 0.9999 \\ 3.00 & -3.76 & 5.42 & 0.9999\end{array}$




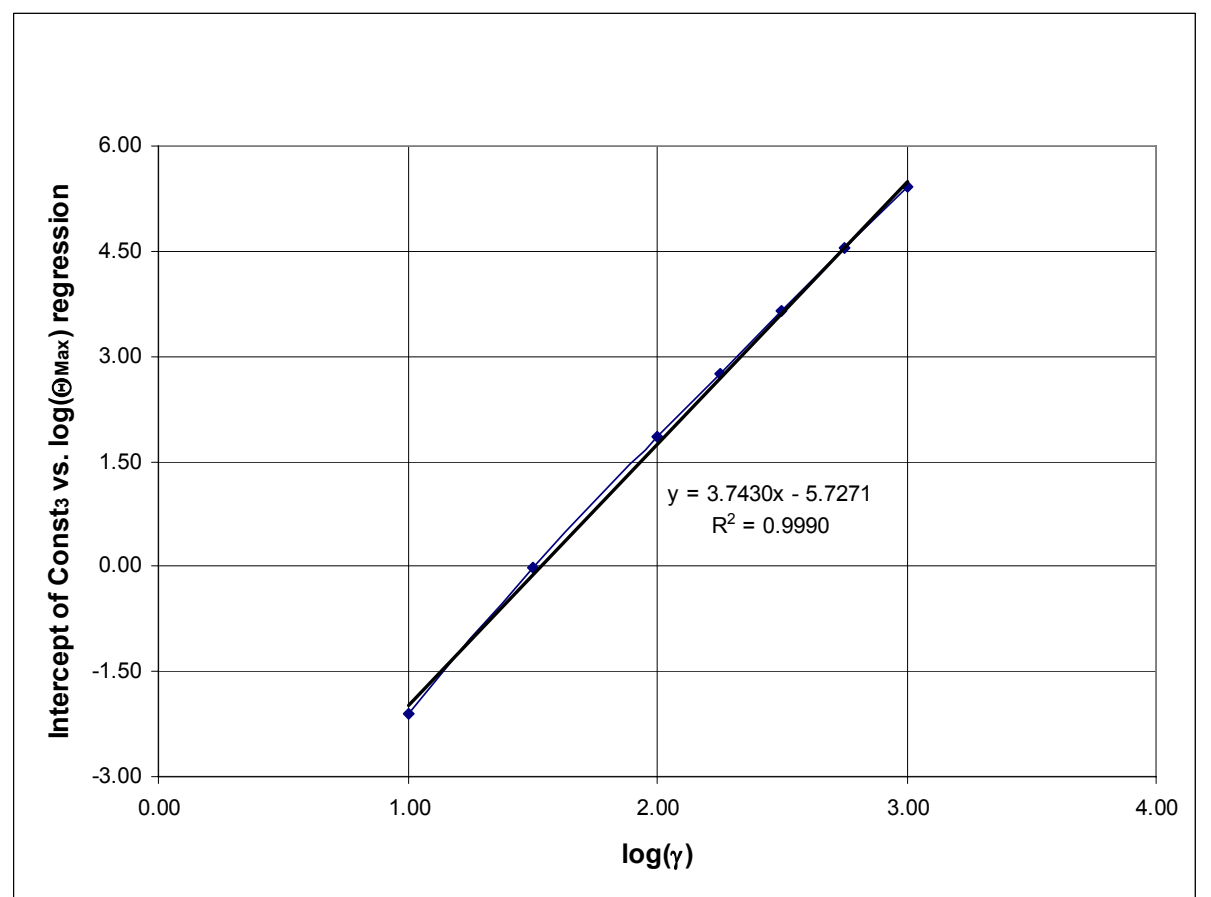

Figure 26. Correlating Coonst $t_{3}$ to $\log (\gamma)$

With these relations in mind, we can write

$$
\text { Const }_{3}=-(3.76) \log \left(\Theta_{\text {Max }}\right)+(3.74) \log (\gamma)-5.73
$$

Or, accepting that 3.76 and 3.74 are essentially the same number,

$$
\text { Const }_{3}=(3.75)\left(\log \left(\frac{\gamma}{\Theta_{\text {Max }}}\right)-1.5\right)
$$

When $\log \left(\gamma / \Theta_{\text {Max }}\right)$ is less than 1.5, then Const $_{3}$ is negative and equation 44 cannot be evaluated. Looking at various versions of the plot in Figure 25, we suggest that $\log \left(\gamma / \Theta_{\text {Max }}\right)$ should be at least 3 for equation 44 to work with reasonable accuracy. For $\log \left(\gamma / \Theta_{\text {Max }}\right)$ values less than 3, a similar equation using a $\Phi^{-2 / 3}$ dependence might be derived. For practical reactive barriers, $\log \left(\gamma / \Theta_{\text {Max }}\right)$ should always be at least 3 .

Knowing all our constants, we can now conclude

$$
\begin{aligned}
\tau_{K}= & \Theta_{\text {Max }}+\frac{1}{6}+\frac{2}{\pi^{2}} \sum_{n=1}^{\infty} \frac{(-1)^{n}}{\boldsymbol{n}^{2}} \exp \left(-\boldsymbol{n}^{2} \pi^{2} \tau_{K}\right) \\
& +\left[\frac{\gamma}{2}-\frac{2}{\pi^{2}} \sum_{n=1}^{\infty} \frac{(-1)^{n}}{\boldsymbol{n}^{2}} \exp \left(-\boldsymbol{n}^{2} \pi^{2} \tau_{K}\right)\right] \operatorname{erfc}\left(3.75\left(\log \left(\frac{\gamma}{\Theta_{\text {Max }}}\right)-1.5\right) \Phi^{-0.5}\right)
\end{aligned}
$$

\section{Discussion}

Our non-particulate model suggests that the leakage of a film varies inversely with $\Phi$ and directly with $\gamma^{2}$. Not only does this confirm our original hypothesis that the leakage is greater when the reaction rate is smaller, but it also confirms our experimental observation that the leakage depends strongly on the amount of scavenger in the membrane. 
In fact, equation 37 shows that the leakage actually increases linearly with the amount of scavenger (since both $\gamma$ and $\Phi$ vary linearly with the amount of scavenger). However, the time before that leakage is realized also increases linearly with the amount of scavenger, so we can improve a barrier (increasing its lag time) even as we increase its leakage. This diminishes the usefulness of the parameter. A suitable alternative may be the quotient of the leakage and the membrane loading, $\Lambda / \gamma$. This parameter is independent of the amount of scavenger and hence of the lag time. This independence allows the barrier designer to use it as a separate measure of barrier quality without factoring it in with the lag time in some arcane formula.

Our kill time analysis suggests a "Window of Opportunity" where small changes in the reaction rate correspond to large changes in barrier performance. We found that the location of this window depends not only on the kill concentration $\Theta_{\text {Max }}$, which we might expect from Figure 22, but also on the membrane loading $\gamma$, which we might not expect when looking at Figure 21. Equation 47 says that the higher $\Theta_{\text {Max }}$ is (i.e. the more solute we can tolerate), the lower $\Phi$ can be without sacrificing the barrier's usefulness. This makes sense since a larger $\Theta_{\operatorname{Max}}$ would require a longer time to reach, and hence allow more time for reaction.

It is not as obvious why a larger $\gamma$ would require a larger $\Phi$ to maintain effectiveness. One might think that with more scavenger, the reaction is already going faster, and so the rate constant would not need to be as large. In some sense, this is true. A membrane with three times more scavenger will have a longer kill time even if its reaction rate is an order of magnitude smaller (assuming it's not in the unreactive plateau). This, however, is not the point. The purpose of locating the "Window of Opportunity" is to determine if we are using what scavenger we have to its full potential. Equation 47 tells us that the more scavenger we have, the harder it is (i.e., where the faster it must react) to use all of it to its full potential.

In this light, it makes more sense. Imagine a membrane on the fast-reaction plateau (i.e. an increase in reaction rate yields little change in $\tau_{\mathrm{K}}$ ). We now triple the amount of scavenger in the membrane. If the reaction were truly instantaneous, this would nearly triple $\tau_{\mathrm{K}}$ (at small $\left.\Theta_{\text {Max }}\right)$. This full increase will not be realized in our membrane, however, because the added scavenger will require an added amount of time to react. While the membrane will still perform much better than it did without the extra scavenger, there is now room for us to improve the membrane even further by increasing the reaction rate and shortening this increased reaction time. We are no longer on the fast-reaction plateau; there is still some room to climb. Figure 21 demonstrates this well. We see that the films with the most scavenger have by far the longest kill times. They also, however, have the most increase in $\tau_{\mathrm{K}}$ at large $\Phi$.

These insights gleaned from equations 37 and 47 may be striking, but perhaps more striking is the fact that an identical analysis using the particulate model gives us the same results. The leakage still varies with $\gamma^{2}$ and $\Phi^{-1}$. The kill time expression is exactly the same, save for replacing the ' 3.75 ' with a ' 2.1 '. The leakage prefactor also decreased, from 5 to 1.3 . Both changes are consistent with having a $\Phi$ whose effect is 3 times greater than in the non-particulate model. Recall that in equation 4.72, a factor of 3 appears in front of $\Phi$ in the reaction term, giving it that greater effect.

The fact that the exact same relationships appear for two different kinetic models gives us hope that these relationships are independent of kinetics. If the kinetics, from which the nonlinear terms arise, are not important, there may be an analytical pathway to these relationships.

While our experimental results could not quantify the breakthrough curves precisely enough to build mathematical correlations, our modeling surpassed our expectations. Now, like with so many other models, we must wait for experimental ability to catch up. 


\section{Diffusion of Mobile Products in Reactive Barrier Membranes}

While the breakthrough of reactive solutes has been carefully investigated above, the fate of any reaction products has not received attention. Such reaction products may be mobile or immobile. The mobile products, which are of more interest, may or may not be desirable. For example, dissolution of an immobilized chemical from within a polymer membrane could be used for the controlled release of agricultural chemicals (Rudzinski et al., 2003; Mishra et al., 2004; Zhao et al., 2005) or pharmaceuticals (Parejo et al., 1998; Petrato et al., 2002; Bravo et al., 2002). In environmental applications relevant to this work, the destruction of one pollutant may result in the production of another pollutant which must be monitored. The origin of the reaction products may be the immobilized reactive material or the solute. That is, transformation of the solute or dissolution of the reactive material can both produce products. Whether the product originates from the solute or the reactive material, the diffusion behavior and concentration profile development should be similar as long as the product is mobile in the membrane. This study investigates the diffusion of mobile products in a reactive membrane system both theoretically and experimentally. In the modeling studies, a diffusion-reaction equation is solved analytically, and a novel equation to describe the concentration of the product is derived. The equation is also solved numerically and compared with the analytical solution. Experimental verification is performed in a diaphragm cell by challenging a zinc oxide ( $\mathrm{ZnO})$-laden PVA membrane with hydrochloric acid $(\mathrm{HCl})$. Finally, the experimental data is compared to the analytical solution.

\section{Theory}

To describe the diffusion of products formed by reaction within membranes, we assume two volumes separated by the reactive membrane. One "upstream cell" volume $\left(V_{u p}\right)$ contains a high concentration of a reactive solute $(\mathrm{HCl})$, and the other "downstream cell" volume $\left(V_{\text {down }}\right)$ initially contains no solute. The membrane, which is permeable to $\mathrm{HCl}$, contains reagent particles $(\mathrm{ZnO})$ that will react with the penetrating $\mathrm{HCl}$. These particles are small relative to the membrane thickness. In this situation, $\mathrm{HCl}$ diffuses into the membrane and is consumed by the reaction:

$$
2 \mathrm{HCl}+\mathrm{ZnO} \rightarrow \mathrm{ZnCl}_{2}+\mathrm{H}_{2} \mathrm{O}
$$

Upon this reaction, soluble zinc chloride $\left(\mathrm{ZnCl}_{2}\right)$ is released and starts to diffuse. The focus of this work is the prediction of the concentration of this product in the two cells as a function of time.

\section{The Reactive Solute}

To model the diffusion of the product, it is first necessary to model the penetration of $\mathrm{HCl}$ into the membrane and the resulting dissolution of $\mathrm{ZnO}$. For simplicity, we consider a uniform distribution of $\mathrm{ZnO}$ in the membrane and a constant concentration of $\mathrm{HCl}$ in the upstream cell. If the $\mathrm{ZnO}$ particle size is significantly smaller than the membrane thickness, we can treat the ZnO-PVA composite as a homogeneous phase:

$$
\frac{L}{d_{p}}>>1
$$

where $L$ is the membrane thickness and $d_{p}$ is the $\mathrm{ZnO}$ particle diameter. We also assume that the reaction is irreversible and much faster than diffusion (i.e., large second Damköhler number, $D_{n}$ ): 


$$
D_{n}=\frac{L^{2}}{D_{l} t_{r}}>>1
$$

where $D_{l}$ is the diffusion coefficient of $\mathrm{HCl}$, and $t_{r}$ is a reaction time. In this limit, $\mathrm{HCl}$ and $\mathrm{ZnO}$ can not coexist, and there is a clear boundary between the $\mathrm{ZnO}$-depleted zone and the ZnO-laden zone in the reactive membrane. This boundary where $\mathrm{HCl}$ meets $\mathrm{ZnO}$ is the "reaction front". It is also assumed that the reaction front moves slowly relative to the diffusion of $\mathrm{HCl}$ in the depleted zone. The quantitative expression for this assumption is:

$$
\frac{\text { Diffusion rate of the reactive solute }}{\text { Advancement rate of the reaction front }} \sim \frac{C_{20}}{v H_{1} C_{1 u p}}>>1
$$

where $C_{20}$ is the initial concentration of $\mathrm{ZnO}, v$ is the stoichiometric coefficient of reaction $(0.5$ in this case), $H_{l}$ is the membrane-water partitioning coefficient of $\mathrm{HCl}$, and $C_{l u p}$ is the concentration of $\mathrm{HCl}$ in the upstream cell. As a result, a mass balance on the volume where the $\mathrm{ZnO}$ is depleted is simply:

$$
\frac{d}{d t}\left(\ell A C_{20}\right)=j_{1} A v=\frac{P_{1} C_{1 u p} A v}{\ell}
$$

where $t$ is time, $\ell$ is the thickness of the depleted zone and also the location of the reaction front, $A$ is the membrane cross-sectional area available to diffusion, $j_{l}$ is the flux of $\mathrm{HCl}$ into the $\mathrm{ZnO}-$ laden zone, and $P_{1}$ is the permeability of the membrane for $\mathrm{HCl}\left(P_{1}=H_{1} \times D_{1}\right)$. Equation 5 is subject to the initial condition:

$$
\ell=0 \text { at } t=0
$$

Integrating Eq. 52, we find:

$$
\ell=\sqrt{\frac{2 P_{1} C_{1 u p} v t}{C_{20}}}
$$

The depleted zone grows until it reaches all the way across the membrane (i.e., $\ell=L$ ), and $\mathrm{HCl}$ starts to diffuse into the downstream cell. The time when this occurs is known as the lag time $\left(t_{\text {lag }}\right)$. Using Eq. 54, the lag time can be expressed as:

$$
t_{\text {lag }}=\frac{C_{20} L^{2}}{2 v P_{1} C_{\text {lup }}}
$$

which we have seen before as Eq. 3. To be more accurate, the lag time for a non-reactive membrane $\left(\frac{L^{2}}{6 D_{1}}\right)$ could be added to Eq. 55. Omitting this additional term simplifies the model and is acceptable when the lag time for a reactive membrane is larger than that for a corresponding non-reactive membrane by an order of magnitude or more, which is true for the experiments conducted in this study.

The $\mathrm{HCl}$ concentration in the downstream cell $\left(C_{\text {ldown }}\right)$ is zero for $t \leq t_{\text {lag }}$, and it increases according to the following equation when $t>t_{\text {lag }}$ :

$$
C_{\text {ldown }}=\frac{P_{1} A C_{\text {lup }}}{L V_{\text {down }}}\left(t-t_{\text {lag }}\right)
$$

which was previously presented as equation 1 . 


\section{Diffusion of Reaction Products}

The above equations, particularly Eq. 54, are the basis for modeling the concentration of the mobile product within the membrane $\left(C_{3}\right)$. We propose the following diffusion equation:

$$
\frac{\partial C_{3}}{\partial t}=D_{3} \frac{\partial^{2} C_{3}}{\partial x^{2}}+\frac{P_{1} C_{1 u p} v}{x} \delta(x-\ell)
$$

where $x$ is the distance into the membrane ( $x=0$ at the upstream end), $D_{3}$ is the diffusion coefficient of the mobile product, and $\delta$ is the Dirac delta function. This equation describes the transport of the mobile product away from a source located at the reaction front. The reaction front moves as described by Eq. 54. Intuitively, the concentration of the product should be higher near the moving front, forming a peak from which the product diffuses in both directions as depicted in Fig. 27. Equation 10 is subject to the following initial and boundary conditions:

$$
\begin{array}{lll}
t=0 & \text { all } x & C_{3}=0 ; \quad C_{3 \text { down }}=0 ; \quad C_{3 \text { up }}=0 \\
t>0 & x=0 & C_{3}=H_{3} C_{3 \text { up }} \\
t>0 & x=L & C_{3}=H_{3} C_{3 \text { down }}
\end{array}
$$

where $H_{3}$ is the membrane-water partition coefficient of the product, and $C_{3 \text { down }}$ and $C_{3 u p}$ are concentration of the product in the downstream and upstream cells, respectively.

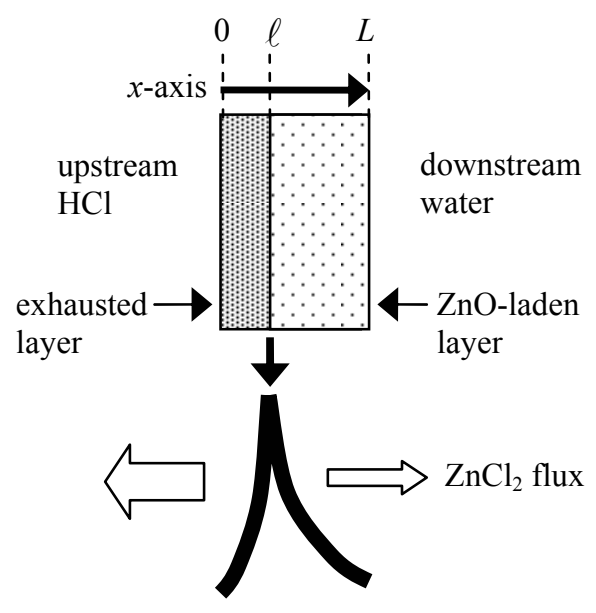

Figure 27. Conceptual drawing of the reactive membrane (top) and concentration profile of the mobile reaction product (bottom). The dashed line indicates the location of the concentration peak which coincides with the location of the reaction front. The peak advances toward the downstream cell with time.

After the reactive material is exhausted (i.e., $t>t_{\text {lag }}$ ), Eq. 57 is not valid because $\mathrm{ZnCl}_{2}$ is no longer produced. The equation now reduces to:

$$
\frac{\partial C_{3}}{\partial t}=D_{3} \frac{\partial^{2} C_{3}}{\partial x^{2}}
$$

The same boundary conditions (Eqs. 59 and 60) apply, but the initial condition corresponds to the concentration profile determined from Eq. 57 at $t=t_{\text {lag. }}$.

To derive an analytical solution to the differential equations, we made a pseudo-steady state assumption that advancement of the reaction front is slow compared with diffusion of the product. Quantitatively, this criterion is expressed by the following equation: 


$$
\frac{\text { Diffusion rate of the product }}{\text { Advancement rate of the reaction front }} \sim \frac{C_{20} D_{3}}{v P_{1} C_{1 u p}}>>1
$$

This assumption leads to the product concentration profiles shown in Fig. 28. These profiles are straight and have a peak located at the reaction front.
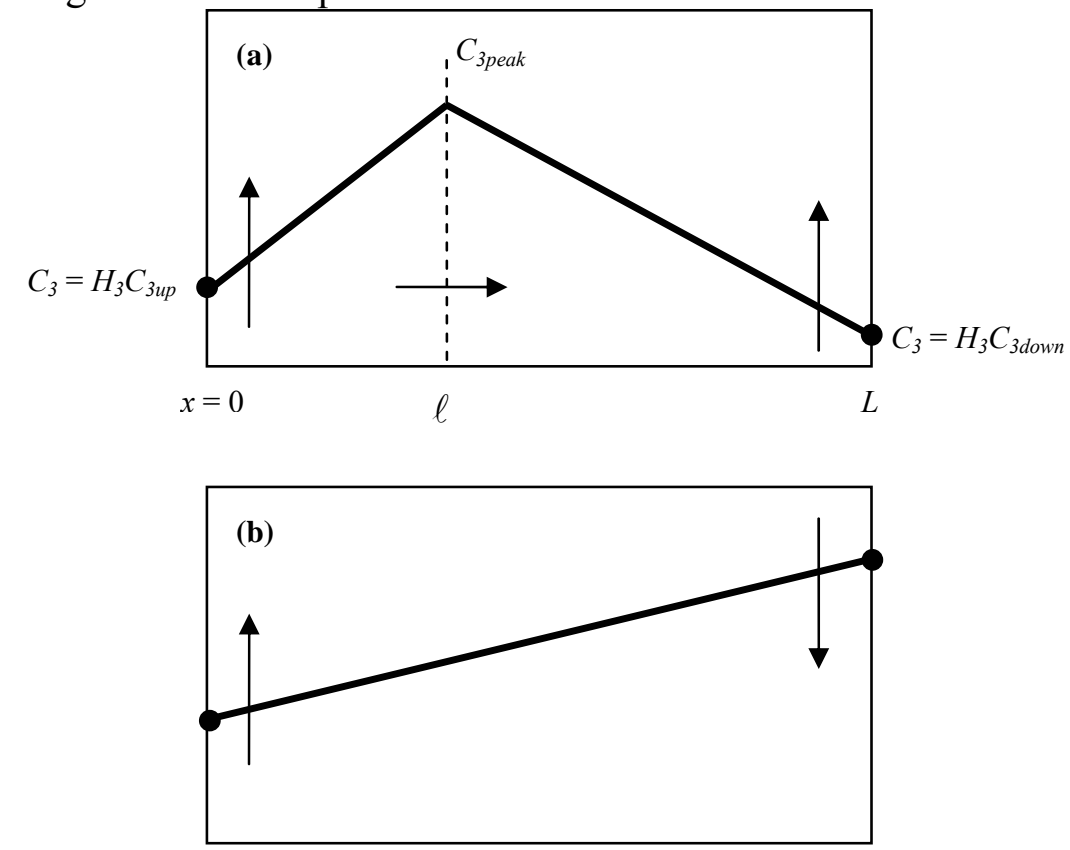

Figure 28. Simplified, linear concentration profiles of the mobile product used to derive the analytical solution: (a) before breakthrough when a reaction front exist and (b) after breakthrough. Arrows indicate the direction the change in concentration or movement of the reaction front.

The concentration of the product in the downstream cell increases as follows:

$$
D_{3} \frac{C_{3 \text { peak }}-H_{3} C_{3 \text { down }}}{L-\ell} A=V_{\text {down }} \frac{d C_{3 \text { down }}}{d t}
$$

where $C_{3 p e a k}$ is the peak height. The independent variable time is replaced by the peak location $(\ell)$ using Eq. 54:

$$
d t=\frac{C_{20} \ell}{P_{1} C_{1 u p} v} d \ell
$$

A mass balance on the product at the reaction front gives:

$$
\frac{P_{l} C_{\text {lup }} v}{\ell}=D_{3} \frac{C_{3 p e a k}-H_{3} C_{3 d \text { own }}}{L-\ell}+D_{3} \frac{C_{3 p e a k}-H_{3} C_{3 u p}}{\ell}
$$

Finally, an overall mass balance of the product yields:

$$
C_{20} A \ell=V_{\text {down }} C_{3 \text { down }}+V_{u p} C_{3 u p}+\frac{C_{3 \text { peak }}+H_{3} C_{3 u p}}{2} A \ell+\frac{C_{3 \text { peak }}+H_{3} C_{3 \text { down }}}{2} A(L-\ell)
$$

When Eqs. 65 and 66 are used to replace $C_{3 \text { peak }}$ in Eq. 63as a function of $C_{3 \text { down }}$ and $\ell$, this problem reduces to a first-order ordinary differential equation with respect to $C_{3 \text { down }}$ and $\ell$. Integration of this differential equation gives: 


$$
\begin{aligned}
& C_{3 \text { down }}=\phi\{1-\exp (-\varepsilon t)\}+\eta \exp (-\varepsilon t) \int_{0}^{t} t^{0.5} \exp (\varepsilon t) d t \\
& C_{3 \text { up }}=\frac{C_{20} \ell-\frac{P_{1} C_{1 u p} v(L-\ell)}{2 D_{3}}-\beta C_{3 \text { down }}}{\alpha}
\end{aligned}
$$

where, $\alpha, \beta, \varepsilon, \phi$, and $\eta$ are constants:

$$
\begin{aligned}
& \alpha=\frac{V_{u p}}{A}+\frac{H_{3} L}{2} \\
& \beta=\frac{V_{\text {down }}}{A}+\frac{H_{3} L}{2} \\
& \varepsilon=\left(\frac{\beta}{\alpha}+1\right) \frac{A H_{3} D_{3}}{V_{\text {down }} L} \\
& \phi=\frac{P_{1} C_{\text {lup }} V V_{u p}}{(\alpha+\beta) A H_{3} D_{3}} \\
& \eta=\frac{1}{\alpha}\left(C_{20}+\frac{P_{1} C_{\text {lup }} v}{2 D_{3}}\right) \sqrt{\frac{2 P_{1} C_{\text {lup }} v}{C_{20}}} \frac{A H_{3} D_{3}}{V_{\text {down }} L}
\end{aligned}
$$

This result is valid as long as there is $\mathrm{ZnO}$ in the membrane. In other words, it is valid until $\mathrm{HCl}$ breaks through.

After the breakthrough of the penetrating solute, the concentration of the mobile product in the upstream cell changes as follows:

$$
\frac{C_{3 d o w n}-C_{3 u p}}{L} A_{3} D_{3}=V_{u p} \frac{d C_{3 u p}}{d t}
$$

Because there is no more production of the product, its total mass is constant:

$$
C_{20} A L=V_{\text {down }} C_{3 \text { down }}+V_{\text {up }} C_{3 u p}+\frac{C_{3 \text { down }}+C_{3 u p}}{2} A H_{3} L
$$

When Eq. 75 is substituted into Eq. 74, a first-order ordinary differential equation with respect to $C_{3 u p}$ and $\ell$ is obtained. Solving this equation results in:

$$
\begin{aligned}
& C_{3 u p}=\frac{C_{20} L}{\alpha+\beta}-\left(\frac{C_{20} L}{\alpha+\beta}-C_{3 u p} \mid t_{\text {tag }}\right) e^{-\frac{A H_{3} D_{3}}{V_{\text {up }} L}\left(\frac{\alpha}{\beta}+1\right)\left(t-t_{\text {lag }}\right)} \\
& C_{3 \text { down }}=\frac{C_{20} L-\alpha C_{3 u p}}{\beta} \\
& \left.C_{3 u p}\right|_{\text {tag }}=\frac{C_{20} L-\beta\left[\phi\left\{1-\exp \left(-\varepsilon t_{\text {lag }}\right)\right\}+\eta \exp \left(-\varepsilon t_{\text {lag }}\right) \int_{0}^{t_{\text {lag }}} t^{0.5} \exp (\varepsilon t) d t\right]}{\alpha}
\end{aligned}
$$

These results complete the approximate, analytical solution.

Experimental data are fit to these equations according to the following procedure. First, a breakthrough curve for $\mathrm{HCl}$ is fit to Eqs. 55 and 56 to obtain $P_{1}$. Using this $P_{1}$, concentrationtime curves for $\mathrm{ZnCl}_{2}$ in the downstream and upstream cells are simultaneously fit to Eqs. 67 and 77 and Eqs. 68 and 76, respectively, which determines $D_{3}$ and $H_{3}$ as fitting parameters.

For a special case where $V_{\text {down }}$ and $V_{u p}$ are significantly large, $C_{3 \text { down }}$ and $C_{3 u p}$ can be assumed to be almost zero. It follows that Eq. 65 reduces to: 


$$
\frac{P_{1} C_{1 u p} v}{\ell}=D_{3} \frac{C_{3 p e a k}}{L-\ell}+D_{3} \frac{C_{3 p e a k}}{\ell}
$$

Transformation of Eq. 79 gives $C_{3 p e a k}$ as a function of $\ell$ :

$$
C_{3 p e a k}=\frac{P_{1} C_{1 u p}(L-\ell) v}{L D_{3}}
$$

Equation 80 implies that the product peak will reduce to zero at the moment of the membrane breakthrough. After the breakthrough, therefore, there is no flux of $\mathrm{ZnCl}_{2}$ into the downstream or upstream cells. The fluxes of $\mathrm{ZnCl}_{2}$ into the downstream cell $\left(j_{3 \text { down }}\right)$ and upstream cell $\left(j_{3 u p}\right)$ are:

$$
\begin{aligned}
& j_{3 \text { down }}=\frac{P_{1} C_{1 \text { lup }} v}{L} \\
& j_{3 \text { up }}=\frac{P_{l} C_{1 \text { lup }}(L-\ell) v}{L \ell}
\end{aligned}
$$

The cumulative mass of $\mathrm{ZnCl}_{2}$ in the downstream cell $\left(M_{3 \text { down }}\right)$ and upstream cell $\left(M_{3 u p}\right)$ can be obtained by the following integration:

$$
\begin{gathered}
M_{3 \text { down }}=\int_{0}^{t_{\text {lag }}} j_{3 \text { down }} A d t=\frac{P_{1} C_{1 u p} A v}{L} t_{\text {lag }}=\frac{P_{1} C_{1 u p} A v}{L} \frac{C_{20} L^{2}}{2 P_{1} C_{1 u p} v}=\frac{C_{20} L A}{2} \\
M_{3 \text { up }}=\int_{0}^{t_{\text {lag }}} j_{3 u p} A d t=\int_{0}^{t_{\text {lag }}} \frac{P_{1} C_{1 u p}(L-\ell) A v}{L \ell} d t=\int_{0}^{L} \frac{P_{1} C_{1 u p}(L-\ell) A v}{L \ell} \frac{C_{20} \ell}{P_{1} C_{1 u p} v} d \ell \\
=\int_{0}^{L} \frac{C_{20}(L-\ell) A}{L} d \ell=\frac{C_{20} L A}{2}
\end{gathered}
$$

As a complement to the approximate, analytical solution, Eq. 57 was solved numerically without any assumptions. The partial differential equation was converted to a difference equation based on the finite difference method by dividing the membrane dimension $L$ into at least 100 grids. The time increment was chosen to be $\Delta t=0.001 \mathrm{~s}$ or smaller. The equation used is:

$$
C_{3}[n+1, k]=C_{3}[n, k]+D_{3} \frac{C_{3}[n, k+1]-2 C_{3}[n, k]-C_{3}[n, k-1]}{\Delta x^{2}} \Delta t+\frac{\operatorname{Pr}[n]}{\Delta x} \Delta t
$$

where $\Delta x$ and $\Delta t$ are distance and time increments, and $n$ and $k$ are indexes for $t$ and $x$ (i.e., $x=$ $k \Delta x$ and $t=n \Delta t$ ), respectively. The moving source term $\operatorname{Pr}$ is:

$$
\operatorname{Pr}[n]=\frac{P_{1} C_{1 u p} v}{\ell[n]}(\text { At } x=\ell[\mathrm{n}] \text { and } \text { before breakthrough) } \text { or } 0 \text { (otherwise) }
$$

Note that the delta function term in Eq. 57 is expressed by $\frac{\operatorname{Pr}[n]}{\Delta x}$. Equation 85 was solved using the Explicit Euler method.

Parameters obtained from fitting of the experimental data to the analytical solution were used for this numerical computation. The numerical solution is then compared with the analytical solution for consistency.

\section{Breakthrough Experiments}

In this study, both the breakthrough of $\mathrm{HCl}$ across a barrier membrane containing $\mathrm{ZnO}$ and the resulting $\mathrm{ZnCl}_{2}$ concentrations in the upstream and downstream solutions were measured. Fig. 29 shows a typical breakthrough curve. The concentration of acid is near zero until the breakthrough and then rises linearly. The lag time was 83 min, while a typical lag time for PVA 
membrane of this thickness without $\mathrm{ZnO}$ is $1 \mathrm{~min}$. This result demonstrates the large effect of $\mathrm{ZnO}$ addition on extending the lag time and is consistent with past studies (Yang et al., 2001; Nuxoll, 2003). No measurable decrease of $\mathrm{pH}$ in the upstream cell was observed.

Seven diffusion experiments were conducted, and their results are summarized in Table 1. The measurements of the product concentrations are exemplified by Fig. 30. This figure shows concentration vs. time plots for $\mathrm{ZnCl}_{2}$ in the downstream and upstream cells for the same

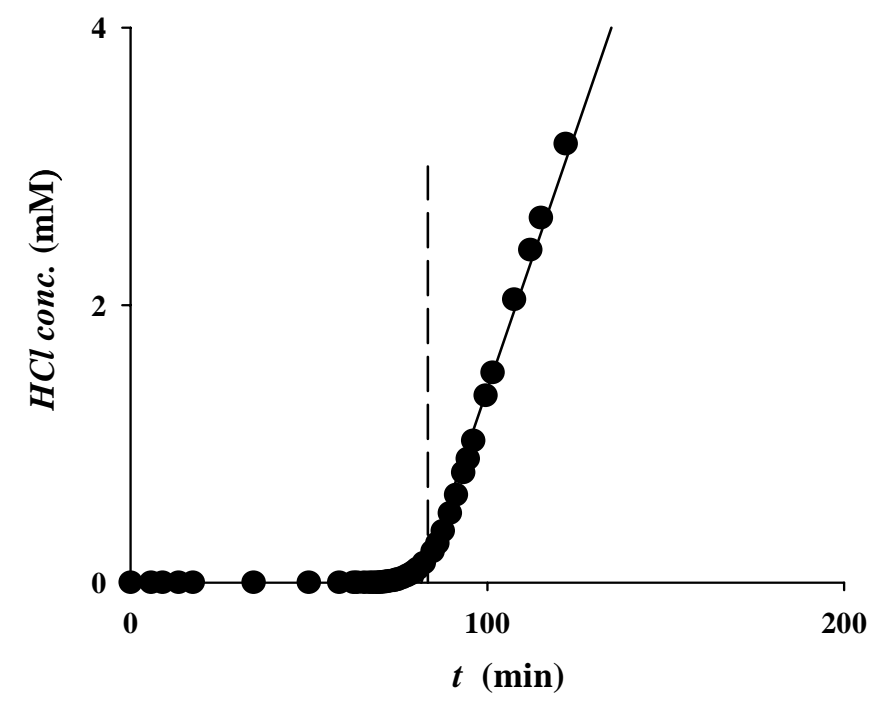

Figure 29. Breakthrough curve of $\mathrm{ZnO} / \mathrm{PVA}$ membrane for $\mathrm{HCl}\left(C_{\text {lup }}=0.01 \mathrm{M}, L=154 \mu \mathrm{m}\right.$, $V_{\text {down }}=12.8 \mathrm{~mL}$ ). The solid line is a regression based on Eqs. 8 and 9. The dotted line indicates the lag time. For reference, a typical lag time for acid through a PVA membrane is $1 \mathrm{~min}$.

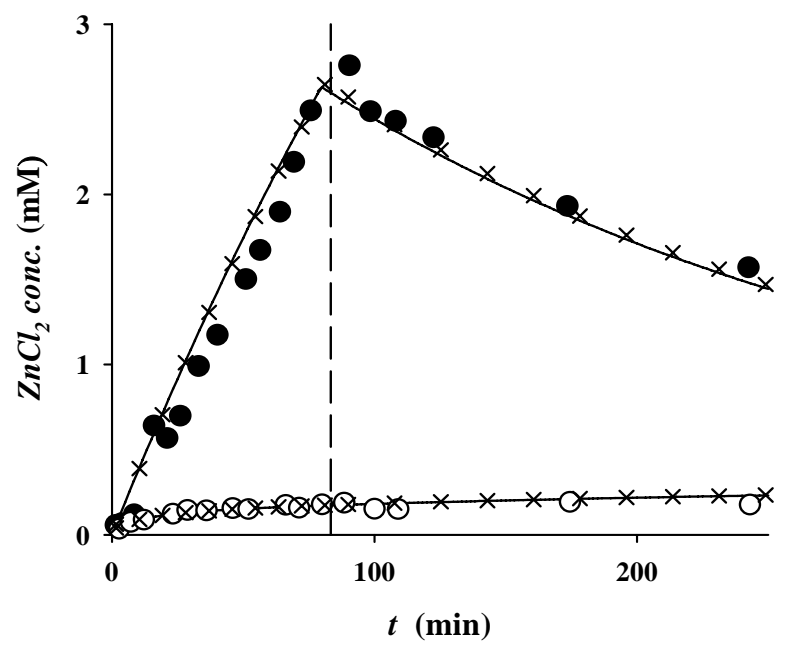

Figure 30. Concentrations of the mobile product in the downstream $(\bullet)$ and upstream cells $(\circ)$. The solid lines are the model fit based on the analytical solution. The numerical solution $(\times)$ using the same parameters almost perfectly matches the analytical solution. The vertical dashed line indicates the lag time from Fig. 4. 
experiment shown in Fig. 29. Note that $C_{3 u p}$ was much smaller than $C_{3 \text { down }}$ for most of the experiment because of the larger volume of the upstream cell. $C_{3 u p}$ increased rapidly at the beginning, followed by a slow increase until the end. On the other hand, $C_{3 \text { down }}$ increased, reached a peak, and then decreased. The peak time coincides with the lag time in Fig. 29. This phenomenon is explained as follows. As long as $\mathrm{ZnO}$ remains in the membrane, there is a concentration peak of the product within the membrane, and $C_{3 \text { down }}$ keeps increasing. After the breakthrough, $\mathrm{ZnO}$ in the membrane is exhausted, and hence no more product is generated. The concentration peak within the membrane quickly vanishes, and the product diffusion is solely due to the remaining concentration gradient. Because $C_{3 \text { down }}$ is higher than $C_{3 u p}$ at the breakthrough, the product naturally starts to diffuse toward the upstream cell (cf. Fig. 28). The experimental data in Fig. 30 and in Table 7 show that the analytical solution can describe the diffusion of the product accurately. The values for the three fitting parameters were determined: $P_{1}=(6.61 \pm 1.58) \times 10^{-10} \mathrm{~m}^{2} / \mathrm{s}, H_{3}=0.676 \pm 0.342$, and $D_{3}=(5.48 \pm 1.04) \times 10^{-10} \mathrm{~m}^{2} / \mathrm{s}$, where the mean values and the standard deviations over the seven experiments are shown. The $\mathrm{R}^{2}$ value for data fitting to a single experiment ranged from 0.92 to 0.99 . Estimated parameters from independent experiments, such as diffusion experiments using pure PVA membranes, could be alternatively used. There is no guarantee, however, that the estimated and fitted values will match because the added immobilized reagent may alter the membrane properties. The trends for $C_{3 u p}$ and $C_{3 \text { down }}$ are correctly predicted, and the lag time coincides with the time when $C_{3 \text { down }}$ reaches a peak. After the breakthrough, however, the decrease in $C_{3 \text { down }}$ tends to be overestimated, while the increase in $C_{3 u p}$ tends to be underestimated.

Table 7. Summary of the diffusion experiments.

\begin{tabular}{lllllllll}
\hline Experiment & $\begin{array}{l}C_{\text {lup }} \\
(\mathrm{M})\end{array}$ & $\begin{array}{l}V_{\text {down }} \\
(\mathrm{mL})\end{array}$ & $\begin{array}{l}V_{\text {up }} \\
(\mathbf{m L})\end{array}$ & $\begin{array}{l}\boldsymbol{C}_{\mathbf{2 0}}{ }^{\mathrm{a}} \\
(\mathrm{M})\end{array}$ & $\begin{array}{l}L \\
(\mu \mathrm{m})\end{array}$ & $\begin{array}{l}t_{\text {lag }} \\
(\mathrm{min})\end{array}$ & $\begin{array}{l}\text { Peak } C_{\text {3down }} \\
(\mathrm{mM})\end{array}$ & Parameter change \\
\hline $\mathrm{A}$ & 0.01 & 12.8 & 254 & 1.00 & 154 & 83.4 & 2.76 & Base experiment \\
$\mathrm{B}$ & 0.01 & 12.2 & 251 & 0.64 & 183 & 60.6 & 1.78 & Low $C_{20}$ \\
$\mathrm{C}$ & 0.01 & 12.2 & 251 & 2.38 & 163 & 169 & 6.49 & High $C_{20}$ \\
$\mathrm{D}$ & 0.01 & 13.1 & 307 & 1.27 & 246 & 190 & 4.12 & Large $L$ \\
$\mathrm{E}$ & 0.01 & 12.6 & 556 & 1.39 & 140 & 43.3 & 2.63 & Large $V_{u p}$ \\
$\mathrm{~F}$ & 0.02 & 12.7 & 314 & 1.37 & 136 & 31.0 & 3.26 & High $C_{\text {lup }}$ \\
$\mathrm{G}$ & 0.005 & 13.5 & 250 & 1.09 & 193 & 179 & 2.82 & Low $C_{\text {lup }}$ \\
\hline
\end{tabular}

${ }^{a}$ This concentration is based on the wet volume of the membrane and the mass balance of zinc after the breakthrough.

As shown in Fig. 30, the numerical and analytical solutions are almost identical. This suggests that the pseudo-steady state assumption made in the analytical solution was appropriate. Note that the decrease in $\Delta x$ or $\Delta t$ did not affect the result, which suggests that $\Delta x=0.01 L$ and $\Delta t$ $=0.001 \mathrm{~s}$ are small enough. Negligible, almost invisible deviations are evidence of the pseudosteady state approximation. For example, Eq. 68 gives a negative value for $C_{3 u p}$ near $t=0$, although the value is close to zero. When $t$ is sufficiently larger than zero, however, the two solutions match almost perfectly.

The development above is for the specific chemical system of $\mathrm{ZnO} / \mathrm{PVA}$ and $\mathrm{HCl}$, solved analytically and numerically for the assumption of a high Damköhler number. We could easily extend our analysis to other reaction stoichiometries by changing the stoichiometric coefficient. With additional effort, we could improve our numerical calculations to explore cases with a small Damköhler number or reaction kinetics. In such cases, it would likely be impossible to derive an analytical solution. A case with relatively small Damköhler numbers for the same 
chemical system $(\mathrm{ZnO} / \mathrm{PVA}$ and $\mathrm{HCl})$ has been studied from the viewpoint of acid breakthrough (Nuxoll, 2003). For the modeling of reaction products, however, we have no experimental results by which we could judge the success of these extensions.

\section{Limiting Cases}

Peak Concentration of the Product. In this section, effects of changing four experimental parameters $\left(C_{20}, L, V_{u p}\right.$, and $\left.C_{1 u p}\right)$ on the maximum value of $C_{3 d o w n}$ are investigated. The results for the seven experiments under different conditions are summarized in Table 7 . The change in the maximum value of $C_{3 \text { down }}$ when these parameters are varied is easily predicted using the model. Figure 31 shows the experimental data as filled circles and the model predictions as solid lines. We tried to change only one parameter at a time, but other parameters also changed slightly. For this reason, minor adjustments were made to all but the "base experiment" using Eq. 67, and the adjusted values are shown in Fig. 31. The vertical dashed lines indicate where a 20\% decrease in $C_{\text {lup }}$ is expected after the breakthrough. Due to the limited volume of the upstream cell, the assumption of a constant $C_{l u p}$ is violated at this point, which forbids use of the analytical equations.

Generally, the peak value of $C_{3 \text { down }}$ clearly increased with increasing $C_{20}$ and $L$, which makes sense because increasing these parameters increases the total mass of $\mathrm{ZnO}$ in the membrane. On the other hand, the peak values are essentially independent of $V_{u p}$ and $C_{l u p}$. Both of these experimental findings were consistent with the model prediction. Again, the model was able to predict the experimental trend accurately. A major cause of the errors is a deviation between the experimental value of $C_{20}$ determined by the mass balance and the corresponding value of $C_{20}$ expected from the experimental lag time.

Cumulative Mass of the Product. Another case of interest is an environmental barrier such as landfill liners for contaminant containment. The destruction of contaminants may produce toxic byproducts, which are undesirable. For example, our studies above with membranes containing iron nanoparticles to prevent carbon tetrachloride breakthrough showed chloroform as one of the reaction products. For this case, considering the large size of the containment unit and its surrounding area, it is reasonable to assume that $V_{d o w n}$, and $V_{u p}$ are infinitely large or to alternatively assume that $C_{3 \text { down }}$ and $C_{3 u p}$ are nearly zero. In this limit, a cumulative mass of the byproduct which escaped from the containment unit becomes our primary concern. A new set of analytical equations (Eqs. 79-84) was derived to compute this cumulative mass $\left(M_{3 \text { down }}\right)$. Equations 83 and 84 clearly show that one half of the byproduct mass will remain in the containment system and the other half will escape, assuming that all of the immobilized chemical initially present in the membrane reacts. Although not shown here, it is straightforward to also derive the corresponding equations for $M_{3 \text { down }}$ and $M_{3 u p}$ as a function of time. 

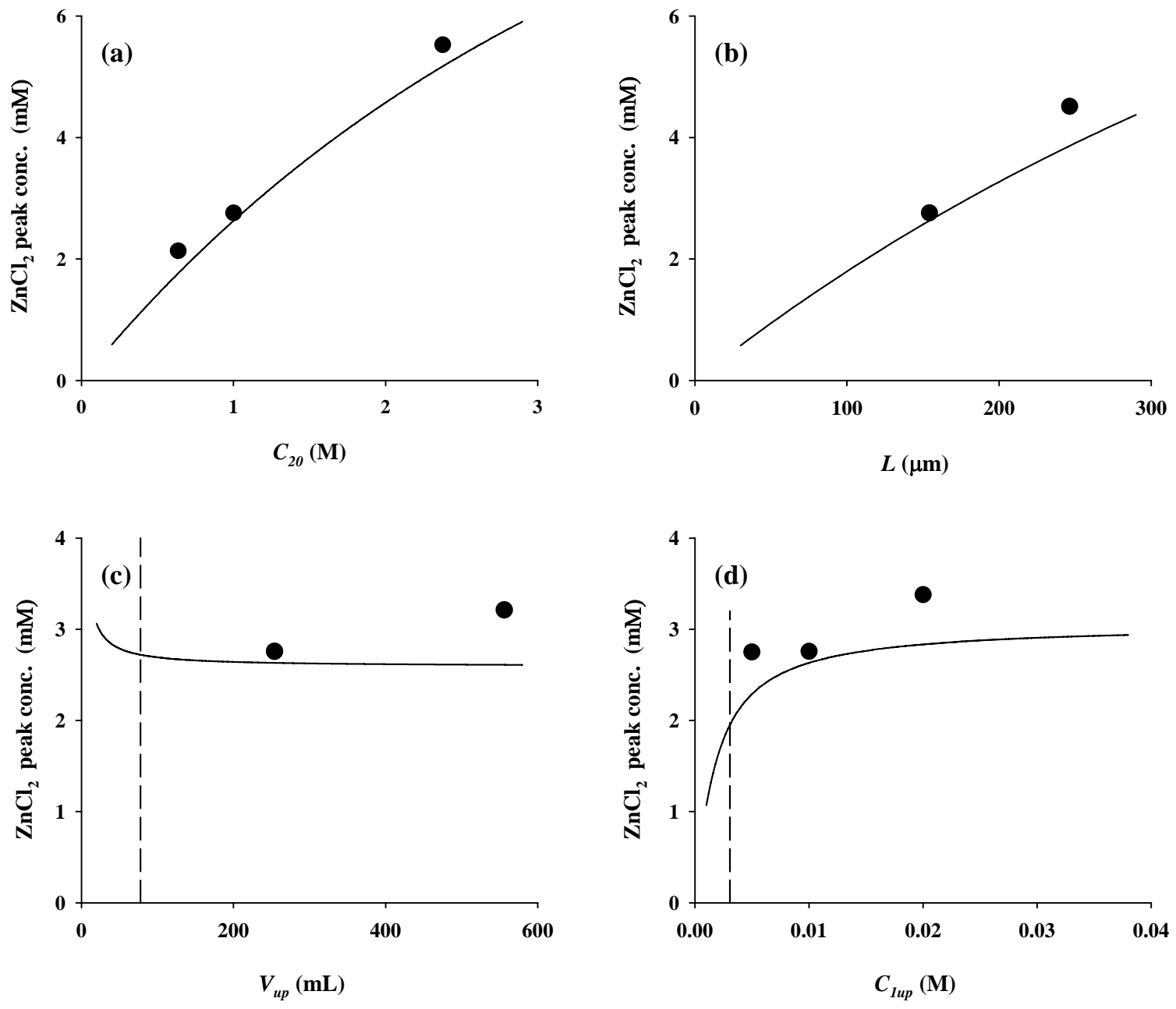

Figure 31. Effects of the four experimental parameters on the peak concentration of $C_{3 \text { down }}$ : (a) $\mathrm{ZnO}$ concentration, (b) membrane thickness, (c) upstream cell volume, and (d) acid concentration in the upstream cell. The solid lines represent the model prediction. Minor adjustments to the experimental data were carried out as explained in the text. The vertical dashed lines represent the points where a $20 \%$ decrease in $C_{1 u p}$ is expected. 


\section{Relevance and Conclusions}

The PVA membranes used in this work were valuable in that they allowed rapid determination of the effects of $\mathrm{Fe}(0)$ and $\mathrm{CST}$ on lag times (i.e. experiments lasting hours to days) as well as a well defined system to test expansions of the theory for reactive membranes. PVA, however, is not a practical material. Hydrated PVA membrane is $50 \%$ water by volume, which makes PVA highly permeable to dissolved species. Thus, a material that is less permeable (such as HDPE) is required in practical applications. PVA will also not stand the $\gamma$-radiation produced by Cs-137 decay. Because the CST concentrates the cesium, it will also concentrate this radiation. This dramatically restricts the choice of polymers. We believe that one possible polymer is polyparaphenyleneterephthalamide (Kevlar) (Lundquist and White, 1999). This material is said to be stable under radiation, but we have not documented this behavior ourselves.

The methods developed developed in studying the PVA membranes, however, are universal. Results can be extrapolated to $\mathrm{Fe}^{0} / \mathrm{HDPE}$ membranes by using appropriate values of $P$ and $L$ and the fraction of iron used. Similar extrapolations may be performed with CST. For example, if a polymer that can stand the radiation can be identified and the theory derived above is valid for impermeable materials, its chances as a successful barrier are excellent. As an example, we imagine that a polyethylene barrier membrane is $1 \mathrm{~mm}$ thick, which is typical for geotextiles. Because transport parameters for cesium through polyethylene are unavailable, those for chloride are used for calculation purposes. The reported diffusion coefficient for chloride through polyethylene is of order $10^{-14} \mathrm{~m}^{2} / \mathrm{sec}$ (Rowe et al., 1995), giving an approximate permeability of $10^{-17} \mathrm{~m}^{2} / \mathrm{sec}$. This latter value corresponds to a transmission rate of about $0.1 \mathrm{mg}$ $\mathrm{mm} / \mathrm{m}^{2}$ day.

We can now estimate the lags across the hypothetical barrier membranes. For the CSTfree membrane, we have from equation (2)

$$
t_{\text {lag }}=\frac{\left(10^{-3} \mathrm{~m}\right)^{2}}{6 \times 10^{-14} \mathrm{~m}^{2} / \mathrm{sec}} \equiv 4600 \mathrm{hrs}
$$

The CST-free membrane will prevent leakage for approximately 6 months. For a membrane with 10 percent CST (by weight) and challenged with a $10^{-3} \mathrm{M}$ cesium solution, we have from equation (5):

$$
t_{\text {lag }}=\frac{\left(10^{-3} \mathrm{~m}\right)^{2}}{2 \times 10^{-17} \mathrm{~m}^{2} / \mathrm{sec}}\left[\frac{\frac{0.1 \mathrm{~g}}{\mathrm{~cm}^{3}}\left(\frac{10^{-3} \mathrm{~mol}}{\mathrm{~g}}\right)}{10^{-6} \mathrm{~mol} / \mathrm{cm}^{3}}\right] \equiv 1.6 \times 10^{5} \mathrm{yrs}
$$

The lag is now many centuries. This dramatic increase results from two factors. The first is the concentration of CST versus the concentration of cesium. The second is the appearance of the permeability $(D H)$ in place of the diffusivity $D$. In other words, for a reactive membrane, the fact that the solubility $H$ is small makes the lag big.

Moving to the results with a practical polymer, we have demonstrated that breakthrough of CT through a HDPE membrane is delayed by adding nano-scale $\mathrm{Fe}^{0}$ and glycerol into the polymer. These idealized experiments, however, are significantly different from actual environments where geomembranes are used. Two major differences are $L$ and $C_{u p}$. Because observation of the breakthrough over a reasonable experimental timescale was desired, small $L$ 
and large $C_{u p}$ values were intentionally chosen to accelerate the experiment. In reality, a minimum thickness of $1.5 \mathrm{~mm}$ is required for a landfill liner, which is almost 10 times thicker than membranes used in this work. A 10 -fold increase in $L$ gives a 100 -fold increase in $t_{\text {lag. The }}$ breakthrough lag times of 6.5 and 13 days which were achieved in this study translate to 1.8 and 3.6 years, respectively. For $C_{u p}$, our use of $\sim 1000 \mu \mathrm{M}$ is extremely high compared to values typically found in contaminated subsurface environments. Typical values are of the order of 10 $\mu \mathrm{M}$ in contaminated groundwater. With $C_{u p}=10 \mu \mathrm{M}$, the lag times will be 180 and 360 years. Although these numbers likely demonstrate a best-case scenario, they suggest that inclusion of reactive material in a containment membrane can dramatically increase $t_{\text {lag }}$ in practical applications.

Chemical complexities also have to be considered. In these experiments with thin membranes, $\mathrm{CF}$ was produced from $\mathrm{CT}$ via reaction with $\mathrm{Fe}^{0}$ in the membrane, but the subsequent reaction of $\mathrm{CF}$ only occurred to a limited extent. CF is also an environmental pollutant, and its production is not desirable. If a 10-fold increase in $L$ is again assumed, the residence time of any contaminant in the membrane will increase by 100 times. Taking into account the difference in reaction kinetics between $\mathrm{CT}$ and $\mathrm{CF}$, this change may provide time for $\mathrm{CF}$ to react within the membrane. Unfortunately, this prediction cannot be confirmed without experiments lasting 2-3 years.

Mechanical properties of the membrane are important factors in installation and durability. Although quantitative measurement was not performed in this work, the $\mathrm{Fe}^{0} / \mathrm{HDPE}$ membranes were more fragile than pure HDPE membranes. Geomembranes are subject to stresses, and mechanical deformation and cracking leads to leakage of contaminants, which is more problematic than diffusion-based leakage. Further improvements in reactive membranes (e.g., polymer selection and additive loading) will be necessary before practical implementation.

Lastly, the models we have developed reveal additional important considerations. For pollutant containment, not just the time to breakthough, but the amount of contaminant that leaks through (prior to or after the lag time) is an important factor that will dictate whether water quality standards are met outside of the containment area. Thus, determining expected 'leakage' or 'kill time' in addition to lag times will be key to evaluating membrane performance.

Additionally, the reaction products may also be of environmental concern, and we have shown that it can be expected that if toxic reaction products are generated (and not consumed) within the membrane, one half of the reaction product mass will be expected to escape. Thus, the reaction chemistry must be thoroughly investigated before application of the technology to a specific pollutant. Carbon tetrachloride is perhaps a worst case scenario in that the product (chloroform) is less reactive that the parent and still of environmental concern. Reactions of chlorinated ethanes and ethylenes are known to lead to non-toxic products and thus are perhaps better candidates for the technology. 


\section{References}

American Public Health Association; American Water Works Association; Water Pollution Control Federation Standard Methods for the Examination of Water and Wastewater : Including Bottom Sediments and Sludges; American Public Health Association: New York, 1998.

Bansleben, D. A.; Opuszko, S.; Speer, D. V. Oxygen Scavenging Composition with Improved Properties and Method of Using Same. 2001, U.S. Patent 6,255,248.

S. A. Bravo, M. C. Lamas, C. J. Salomon, In-Vitro Studies of Diclofenac Sodium ControlledRelease from Biopolymeric Hydrophilic Matrices, J. Pharm. Pharmaceut. Sci 2002, 5, 213.

Crank, J. The Mathematics of Diffusion; Clarendon Press: Oxford, UK, 1975.

Devlin, J. F.; Klausen, J.; Schwarzenbach, R. P. Kinetics of Nitroaromatic Reduction on Granular Iron in Recirculating Batch Experiments. Environ. Sci. Technol. 1998, 32(13), 1941-1947.

Duncalf, B.; Dunn, A. S. Light-Sensitized Crosslinking of Polyvinyl Alcohol by Chromium Compounds. J. Appl. Polym. Sci. 1964, 8, 1763-1776.

Farrell, J.; Kason, M.; Melitas, N.; Li, T. Investigation of the Long-Term Performance of ZeroValent Iron fro Reductive Dechlorination of Trichloroethylene. Environ. Sci. Technol. 2000, 34(3), 514-521.

Gu, D.; Nguyen, L.; Philip, C. V.; Huckman, M. E.; Anthony, R. G. Cs ${ }^{+}$Ion Exchange Kinetics in Complex Electrolyte Solution Using Hydrous Crystalline Silicotitanates, Ind. Eng. Chem. Res. 1997, 36, 5377-5383.

Joo, J. C.; Kim, Y. J.; Nam, K. P. Mass Transfer of Organic Compounds in Dilute Aqueous Solutions into High Density Polyethylene Geomembranes. J. Environ. Eng. 2004, 130(2), 175-183.

Lundquist, S. H.; White, L. R. Method for Removing Metal Ions from Solution with Titanate Sorbents. 1999, U.S. Patent 5,989,434.

Melitas, N.; Chuffe-Moscoso, O.; Farrell, J. Kinetics of Soluble Chromium Removal from Contaminated Water by Zerovalent Iron Media: Corrosion Inhibition and Passive Oxide Effects. Environ. Sci. Technol. 2001, 35(19), 3948-3953.

S. Mishra, J. Bajpai, A. K. Bajpai, Evaluation of the Water Soprtion and Controlled-Release Potential of Binary Polymeric Beads of Starch and Alginate Loaded with Potassium Nitrate as an Agrochemical, J. Appl. Polym. Sci. 94 (2004) 1815. 
Nuxoll, E. E.; Siegel, R. A.; Cussler, E. L. Layered Reactive Barrier Films, J. Membr. Sci. 2005, 252, 29-36.

Nuxoll, E.E. Transient Transport in Reactive Barrier Membranes, Ph.D. Thesis, University of Minnesota, Minneapolis, 2003.

Parejo, C.; Gallardo, A.; SanRoman, J. Controlled Release of NSAIDs Bound to Polyacrylic Carrier Systems, J. Mater. Sci.: Mater. Med. 1998, 9, 803.

Paul, D. R.; Koros, W. J. Effect of Partially Immobilizing Sorption on Permeability and the Diffusion Lag Time. J. Poly. Sci. Poly. Phys. IV, 1976, 675-685.

Petratos, P.B.; Chen, J.; Felsen, D.; Poppas, D. P. Local Pharmaceutical Release from a New Hydrogel Implant, J. Surg. Res. 2002, 103, 55.

Pratt, A. R.; Blowes, D. W.; Ptacek, C. J. Products of Chromate Reduction on Proposed Subsurface Remediation Material. Environ. Sci. Technol. 1997, 31(9), 2492-2498.

Rowe, R. K.; Hrapovic, L.; Kosaric, N. Diffusion of Chloride and Dichloromethane through an HDPE Geomembrane. Geosynthetics Int. 1995, 2(3), 507-536.

Rudzinski, W.E.; Chipuk, T.; Dave, A. M.; Kumbar, S. G.; Aminabhavi, T. M. pH-Sensitive Acrylic-Based Copolymeric Hydrogels for the Controlled Release of a Pesticide and a Micronutrient, J. Appl. Polym. Sci. 2003, 87, 394.

Scancar, J.; Milacic, R.; Strazar, M.; Burica, O. Total Metal Concentrations and Partitioning of $\mathrm{Cd}, \mathrm{Cr}, \mathrm{Cu}, \mathrm{Fe}, \mathrm{Ni}$ and $\mathrm{Zn}$ in Sewage Sludge. Sci. Total Environ. 2000, 250(1-3), 9-19.

Scherer, M. M.; Westall, J. C.; Ziomek-Moroz, M.; Tratnyek, P. G. Kinetics of Carbon Tetrachloride Reduction at an Oxide-Free Iron Electrode. Environ. Sci. Technol. 1997, $31(8), 2385-2391$.

Schrick, B.; Blough, J. L.; Jones, D. A.; Mallouk, T. E. Hydrochlorination of Trichloroethylene to Hydrocarbons Using Bimetallic Nickel-Iron Nanoparticles. Chem. Mater. 2002, 14(12), $5140-5147$.

Schwark, D. W.; Speer, D. V.; McAllister, L. B. Oxygen Scavenging Film with High Slip Properties. 2004, U.S. Patent 20040151934.

Stookey, L. L. Ferrozine - A New Spectrophotometric Reagent for Iron. Anal. Chem. 1970, 42(7), $779-781$.

Siegel, R. A.; Cussler, E. L. Reactive Barrier Membranes: Some Theoretical Observations Regarding the Time Lag and Breakthrough Curves. J. Membr. Sci. 2003, 229(1-2), 33-41. 
US Environmental Protection Agency, Design, Operation, and Closure of Municipal Solid Waste Landfills; US Environmental Protection Agency: Cincinnati, OH, 1994, EPA/625/R94/008.

Van Nice, L. H.; Farlee, R. A Study of the Photochemical Reaction Between Poly(Vinyl Alcohol) and Aqueous Chromate Ion. Polym. Sci. Eng. 1977, 17(6), 359-365.

Wang, C.; Zhang, W. Synthesizing Nanoscale Iron Particles for Rapid and Complete Dechlorination of TCE and PCBs. Environ. Sci. Technol. 1997, 31(7), 2154-2156.

Yang, C.; Nuxoll, E. E.; Cussler, E. L. Reactive Barrier Films. AIChE J. 2001, 47(2), 295-302.

Zhao, J.; Wilkins, R. M. Low Molecular Weight Polylactic Acid as a Matrix for the Delayed Release of Pesticides, J. Agric. Food Chem. 2005, 53, 4076.

Zheng, Z.; Philip, C. V.; Anthony, R. G.; Krumhansl, J. L.; Trudell, D. E.; Miller, J. E. Ion Exchange of Group I Metals by Hydrous Crystalline Silicotitanates, Ind. Eng. Chem. Res. 1996, 35, 4246-4256. 


\section{Personnel Supported}

$\underline{\text { PIs }}$

Dr. William A. Arnold

Dr. Edward L. Cussler

$\underline{\text { Technical Staff }}$

Mr. Dennis Gulden

Graduate Students

Eric E. Nuxoll

Tsutomu Shimotori

Andrew M. Warta

Erin M. Surdo

\section{Acknowledgments}

Additional support was derived from

- A University of Minnesota Graduate School Doctoral Dissertation Fellowship to Tsutomu Shimotori

- Support from NSF-IGERT for Erin Surdo

- A Institute of Hazardous Materials Management grant

- Air Force Office of Scientific Research grant F49620-01-1-0333

- Petroleum Research Fund grant 39083-AC9

- National Science Foundation grant CTS 0322882

\section{Publications}

Eric E. Nuxoll, 2003. Transient transport in reactive barrier membranes. Ph.D. Dissertation, Department of Chemical Engineering and Materials Science, University of Minnesota, Minneapolis, MN.

Tsutomu Shimotori, 2005. Polymer membranes containing zero-valent iron as contaminant barriers. Ph.D. Dissertation, Department of Civil Engineering, University of Minnesota, Minneapolis, MN.

Andrew M. Warta, Ph.D. Dissertation, Department of Chemical Engineering and Materials Science, University of Minnesota, Minneapolis, MN, in progress.

Shimotori, T.; Cussler, E. L. and Arnold, W. A., 2007. Diffusion of Mobile Products in Reactive Barrier Membranes. Journal of Membrane Science, in press. 
Shimotori, T.; Cussler, E. L. and Arnold, W. A., 2006. High-Density Polyethylene Membrane Containing $\mathrm{Fe}^{0}$ as a Contaminant Barrier. Journal of Environmental Engineering-ASCE, 132(7) pp. 803-809.

Warta, A.M., Arnold, W. A. and Cussler, E. L. 2005. Permeable Membranes Containing Crystalline Silicotitanate As Model Barriers For Cesium Ion. Environmental Science and Technology, 39(24) 9738-9743.

Nuxoll, E.E.; Siegel, R.A. Cussler, E.L. 2005. Layered reactive barrier films, Journal of Membrane Science, v. 252, pp. 29-36.

Nuxoll, E.E.; Cussler, E.L. 2005. The third parameter in reactive barrier films. AIChE Journal, v. 51(2), pp. $456-463$.

Shimotori, T.; Nuxoll, E. E.; Cussler, E. L. and Arnold, W. A., 2004. A polymer Membrane Containing $\mathrm{Fe}^{0}$ as a Contaminant Barrier. Environmental Science and Technology, v. 38(7), pp. 2264-2270.

\section{Presentations}

\section{Conference Presentations}

1. Shimotori, T.; Cussler, E. L.; Arnold, W. A. "Diffusion and Release of a Mobile Product in a Reactive Membrane System." Paper presented in American Institute of Chemical Engineers Annual Meeting, San Francisco, CA, Nov. 12-17 (2006).

2. Shimotori, T.; Cussler, E.L.; Arnold, W.A., 2005. Polyethylene membranes containing $\mathrm{Fe}(0)$ as contaminant barriers. Paper presented in Membrane-Based Processes for Water Treatment, Reclamation and Remediation Pacifichem 2005, Honolulu, HI, December 15$20,2005$.

3. Warta, A.M.; Arnold, W.A.; Cussler E.L. Cesium Selective Reactive Barrier Membranes. Poster presented at the $98^{\text {th }}$ Annual Air and Waste Management National Meeting, Minneapolis, MN, June 21-24, 2005.

4. Shimotori T.; Cussler, E.L.; Arnold, W.A., 2004. A novel contaminant barrier: highdensity polyethylene membrane embedded with $\mathrm{Fe}(0)$. Poster presented at the Environmental Sciences: Water Gordon Research Conference, Plymouth, NH, June 27July 2, 2004.

5. Nuxoll, E.E.; Shimotori, T.; Arnold, W.A.; Cussler, E.L. Iron Nanoparticles in Reactive Environmental Barriers. Presented in "Membranes in Environmental Applications," Annual Meeting Archive - American Institute of Chemical Engineers, San Francisco, CA, November 16-21, 2003 (2003), \#84b. 
6. Shimotori, T.; Nuxoll, E.E.; Cussler, E.L.; Arnold, W.A., 2003. Polymer membranes containing iron(0) as contaminant barriers. Presented in "Metal-Organic Interactions in Environmental Systems," Environmental Chemistry Division, American Chemical Society National Meeting, New Orleans, LA, March 23-27, 2003, 43(1), pp. 554-558.

7. Nuxoll, E.E.; Arnold, W.A.; Cussler, E.L. 2002. Kinetic Effects in Reactive Barrier Membranes. Annual Meeting Archive - American Institute of Chemical Engineers, Indianapolis, IN Nov.7, 2002, (2002), pp. 2137-2143.

\section{Invited Presentations for W.A. Arnold}

1. Invited Lecture: Reactive Membrane Barriers for Environmental Applications. Department of Civil and Environmental Engineering, Carnegie Mellon University, Pittsburgh, PA, April 9, 2004.

2. Invited Presentation: Nanoscale Iron Filled Membranes for Toxics Containment. Three Rivers Chapter of the Academy of Certified Hazardous Materials Managers, Pittsburgh, PA, April 8, 2004.

3. Invited Lecture: Development of Reactive Membrane Barriers for Environmental Applications. Department of Civil and Environmental Engineering, University of Iowa, Iowa City, IA, March 5, 2004.

4. Invited Lecture: Development of Reactive Membrane Barriers for Environmental Applications. Department of Civil \& Environmental Engineering, University of IllinoisUrbana-Champaign, Urbana, IL, September 25, 2003.

5. Invited Lecture: Reactive Membrane Barriers for Containment of Subsurface Contamination. Department of Environmental Sciences, Rutgers University, New Brunswick, NJ, November 25, 2002.

6. Invited Presentation: Reactive membrane barriers as landfill liners. 2002 Gordon Research Conference on Membranes: Materials and Processes, New London, NH, August 4-9, 2002.

Invited Presentations for E.L. Cussler

$\underline{2003}$

- "Barrier Membranes," and "Diffusion for Dummies," Robert J. Adler lectures, Case Western Reserve University, Cleveland, OH, April 24

- "Barrier Membranes," "Chemical Product Design," Dean's Lectures, University of Wyoming, Laramie, WY, May 8

- "Barrier Membranes," North Dakota State University, Fargo ND, June 5

- "Barrier Membranes," Gordon Conference on Films and Coatings, New London, NH, July 14 2004

- "Barrier Membranes," St. Anthony Falls Laboratory, University of Minnesota, Jan 21

- "Barrier Membranes," plenary talk, North American Membrane Society, Honolulu, HI, June 30 
- "Barrier Membranes," Cambridge University, UK, Oct 1

- "Barrier Membranes," Eastman Chemical, Kingsport, TN, Oct 6

$\underline{2005}$

- "Barrier Membranes," Arizona State University, Dec 2

$\underline{2006}$

- "Barrier Membranes," “Chemical Product Design,” Johns Hopkins University (Holtz lectures), Dec 2 